Design and Implementation of Color-Shift Keying for Visible Light Communications 


\title{
DESIGN AND IMPLEMENTATION OF COLOR-SHIFT KEYING FOR VISIBLE LIGHT COMMUNICATIONS
}

BY

ERIC MONTEIRO, B.Sc.

\begin{abstract}
A THESIS
SUBMITTED TO THE DEPARTMENT OF ELECTRICAL \& COMPUTER ENGINEERING AND THE SCHOOL OF GRADUATE STUDIES

OF MCMASTER UNIVERSITY
\end{abstract}

IN PARTIAL FULFILMENT OF THE REQUIREMENTS

FOR THE DEGREE OF Master of Applied Science

(c) Copyright by Eric Monteiro, September 2013

All Rights Reserved 
Master of Applied Science (2013)

(Electrical \& Computer Engineering)
McMaster University

Hamilton, Ontario, Canada

TITLE: Design and Implementation of Color-Shift Keying for Visible Light Communications

AUTHOR:

Eric Monteiro

B.Sc., Electrical Engineering, McMaster University, Hamilton ON, Canada

SUPERVISOR:

Dr. Steve Hranilovic

NUMBER OF PAGES: $\quad \mathrm{xx}, 131$ 
I would like to dedicate this thesis to my lovely wife Kristin Monteiro. If it wasn't for her tolerating my temper, helping me cope with stress, and providing amazing editorial support, this work would not exist. 


\section{Abstract}

Color-shift keying (CSK) is a visible light communication (VLC) intensity modulation scheme, outlined in IEEE 802.15.7, that transmits data imperceptibly through the variation of the light color emitted by a red, green, and blue (RGB) light emitting diode (LED). Unlike other intensity modulation schemes, CSK guarantees that the intensity of the luminary will not fluctuate, thus limiting potential human health complications related to flickering light.

In this work, a rigorous design framework for CSK constellations is presented. The benefits of the frame work are that it can optimize constellations while accounting for cross talk between the color communication channels formed by the colored LEDs. Unlike previous works, the method applies the study of colorimetry to optimize higher order CSK constellations such that the luminary functions with a desired operating color, allowing constellations to be designed to meet lighting industry quality metrics.

This work concludes with the implementation of a CSK communication channel for the purposes of measuring the symbol error rate (SER) versus signal to noise ratio (SNR) of CSK constellations. It is demonstrated that the optimized constellations can achieve equal performance to the standardized constellations, outlined in Section 12 of IEEE 802.15.7, while only requiring half the transmitted power. 


\section{Acknowledgments}

Throughout my graduate career, Dr. Steve Hranilovic has been an excellent mentor. While his stubbornness only shadowed my own, I would thank Steve for his immense support in achieving my projects goals. Without Steve's guidance I would likely still be fighting over ten other thesis topics. I would also like to thank my colleagues Danny Vacar and Micheal Taylor for keeping the laboratory social and providing me with excellent technical aid; without them, this work would still be months from completion. 


\title{
Symbols and Notation
}

\author{
E Illuminance (Lux) \\ $E_{h} \quad$ Horizontal Illuminance (Lux) \\ $K_{w} \quad$ Maximum luminous efficacy (Lumens/Watt) \\ L CSK Constraint: Total RGB intensity (Lumens or Amps) \\ $L(\lambda) \quad$ CIE luminosity function (Unitless) \\ $L_{r, g, b} \quad$ CSK Constraint: Peak intensity (Lumens or Amps) \\ $N \quad$ Number of symbols in alphabet \\ $P(\lambda) \quad$ Radiant flux power spectral density (Watts) \\ $P_{a v g} \quad$ Constraint: Average radiant flux (Watts) \\ $P_{\text {peak }} \quad$ Constraint: Peak radiant flux (Watts) \\ $R(\lambda) \quad$ Responsivity (Amps/Watt) \\ $\Phi_{L} \quad$ Luminous Flux (Lumen) \\ $\Phi_{R} \quad$ Radiant Flux (Watt) \\ $\Phi_{r}(t) \quad$ Instantaneous radiant flux (Watts) \\ $\phi_{\mathbf{i}} \quad$ CSK symbol in the normalized design space (Unitless) \\ $\eta_{r, g, b} \quad$ Luminous efficacy (Lumens/Amp) \\ H CSK channel gain matrix (Amps/Lumen)
}




$\begin{array}{ll}\tilde{\mathbf{s}_{\mathbf{i}}} & \text { CSK symbol in the receive intensity space (Amps) } \\ \mathbf{c}_{\mathbf{i}} & \text { CSK symbol in the CIE chromaticity gamut (Unitless) } \\ \mathbf{d}^{*} & \text { Equivalent color point of } \mathbf{d} \text { in normalized design space (Unitless) } \\ \mathbf{d} & \text { RGB intensities required to achieve desired output color (Lumens or Amps) } \\ \mathbf{s}_{\mathbf{i}} & \text { CSK symbol in the transmit intensity space (Lumen) } \\ i_{r}(t) & \text { Instantaneous photo diode output electrical current (Amps) } \\ i_{t}(t) & \text { Instantaneous LED electrical current (Amps) } \\ x, y, z(\lambda) & \text { CIE Color Matching Functions (Unitless) }\end{array}$




\section{Acronyms}

CCT Correlated Color Temperature

CFF Critical Flicker Fusion

CIE International Commission on Illumination

CRI Color Rendering Index

CSK Color-Shift Keying

DAC Digital to Analog Converter

DIN German Institute for Standardization

FCC Federal Communications Commission

Gbps $\quad$ Giga Bits per Second

Gsps $\quad$ Giga Samples per Second

IEC International Electrotechnical Commission

IEEE Institute of Electrical and Electronics Engineers

IM/DD Intensity Modulation and Direct Detection

ISO International Organization for Standardization

JEITA Japan Electronics and Information Technology Industries Association 


\begin{tabular}{|c|c|}
\hline LED & Light Emitting Diode \\
\hline Mbps & Mega Bits per Second \\
\hline MIMO & Multiple Input Multiple Output \\
\hline Msps & Mega Samples per Second \\
\hline OFDM & Orthogonal Frequency Division Multiplexing \\
\hline OMEGA & project hOME Gigabit Access \\
\hline OOK & On-Off Keying \\
\hline PLC & PowerLine Communications \\
\hline PoE & Power over Ethernet \\
\hline $\mathrm{RG}$ & Risk Group \\
\hline RGB & Red Green Blue \\
\hline SNR & Signal to Noise Ratio \\
\hline SNRe & Electrical Signal to Noise Ratio \\
\hline SNRo & Optical Signal to Noise Ratio \\
\hline SPD & Spectral Power Density \\
\hline VLC & Visible Light Communications \\
\hline VLCC & Visible Light Communications Consortium \\
\hline VPPM & Variable Pulse Position Modulation \\
\hline WDM & Wavelength Division Multiplexing \\
\hline $\mathrm{Wi}-\mathrm{Fi}$ & Wireless Fidelity \\
\hline
\end{tabular}




\section{Contents}

Abstract $\quad$ V

Acknowledgments vii

1 Introduction 1

1.1 State of Visible Light Communications . . . . . . . . . . . . . . 3

1.1.1 Visible Light Communications Challenges . . . . . . . . . . 4

1.1.2 Visible Light Communication Applications . . . . . . . . . . . 6

1.2 Thesis Contributions .................... 8

1.3 Outline of Thesis . . . . . . . . . . . . . . . . 11

2 Human Vision, Lighting Standards and Channel Modeling 13

2.1 Human Perception of Light . . . . . . . . . . . . . . . 13

2.1.1 Perception of Intensity . . . . . . . . . . . . . . . . 14

2.1.2 Perception of Color . . . . . . . . . . . . . . . 20

2.1.3 Perception of Flicker .................. 24

2.2 Standards and Constraints . . . . . . . . . . . . 25

2.3 The Visible Light Communications Channel . . . . . . . . . . . . . . 30

2.3.1 Receiver Element: photodiode . . . . . . . . . . . . . 30 
2.3.2 Transmitter Element: Light Emitting Diodes . . . . . . . . . . 31

2.3.3 Channel Model . . . . . . . . . . . . . . . . . 35

2.4 Example of an indoor VLC channel . . . . . . . . . . . . . . . . . 39

2.5 Compatible VLC Modulation Techniques . . . . . . . . . . . . . . . . 42

2.5.1 On-Off Keying . . . . . . . . . . . . . . . . . . . . . . . 42

2.5.2 Variable Pulse Position Modulation . . . . . . . . . . . . . 43

2.5.3 Multiple Sub-Carrier Modulation . . . . . . . . . . . . . 44

2.5.4 Metameric Modulation . . . . . . . . . . . . . . 45

2.5.5 Color-Shift Keying . . . . . . . . . . . . . . . . 45

2.6 Standardized CSK Constellation Design Rules . . . . . . . . . . . . 49

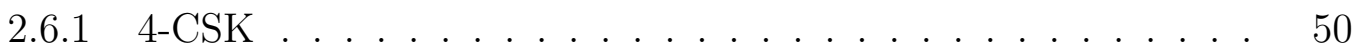

$2.6 .2 \quad 8-\mathrm{CSK} \ldots \ldots \ldots \ldots \ldots$

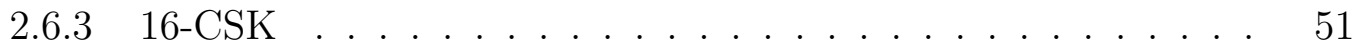

2.7 Conclusions . . . . . . . . . . . . . . . . . . . 52

3 Designing CSK Constellations $\quad 55$

3.1 Optimization Constraints . . . . . . . . . . . . . . 56

3.1.1 Luminous Flux Constraints . . . . . . . . . . . . 56

3.1.2 Chromaticity Constraint . . . . . . . . . . . 60

3.1.3 Modification for constant current . . . . . . . . . . . . 60

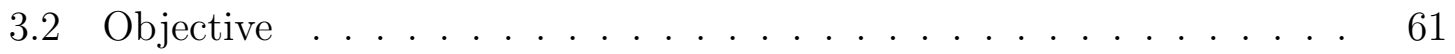

3.3 MATLAB fmincon ..................... 64

3.3.1 Stopping Conditions ... . . . . . . . . . . 65

3.3.2 Starting Point . . . . . . . . . . . . . . . . 67

3.4 Constellation design using fmincon . . . . . . . . . . . . . 67 
3.5 Design Samples . . . . . . . . . . . . . . . . . . . . . . . . . 69

3.6 Hexagonal Lattice Heuristic _ . . . . . . . . . . . . . . . . . . 72

3.6.1 The Heuristic Optimization Process . . . . . . . . . . . . 74

3.7 Constraint Relaxation . . . . . . . . . . . . . . . . . . 77

3.7 .1 Optimization Problem . . . . . . . . . . . . 78

3.7 .2 Design Samples . . . . . . . . . . . . . . . . . . 80

3.8 Conclusions . . . . . . . . . . . . . . . . . . . . . 83

4 Implementation and Characterization of an Experimental CSK Com$\begin{array}{ll}\text { patible Communication System } & 85\end{array}$

4.1 Implementation Details . . . . . . . . . . . . . . . . 86

4.1 .1 Transmitter Elements . . . . . . . . . . . . . . . . . . . . 87

4.1 .2 Receiver Elements . . . . . . . . . . . . . . . . . . . . 91

4.2 System Characterization . . . . . . . . . . . . . . 95

4.2 .1 Bandwidth and Linearity . . . . . . . . . . . . . . . . . 96

4.2 .2 Noise Characterization . . . . . . . . . . . . . . . . 97

4.2 .3 Channel Estimation . . . . . . . . . . . . . . . . . . . 100

4.2.4 Illuminance and Signal to Noise Ratio _. . . . . . . . . . 101

4.3 Conclusions . . . . . . . . . . . . . . . . . . . . . . . . 103

5 Simulated and Experimental Error Performance 105

5.1 Constellation Design for the Implemented CSK Channel . . . . . . 106

5.1 .1 Constraint Formulation . . . . . . . . . . . . . 106

5.1 .2 Designed Constellations _. . . . . . . . . . . . . . . . 109

5.2 Experimental Performance . . . . . . . . . . . . . . . . . . 115 
5.2.1 Measurement Method . . . . . . . . . . . . 115

5.2.2 Experimental Results . . . . . . . . . . . . . . . 119

5.3 SNR versus Illuminance . . . . . . . . . . . . . . . 121

5.4 Conclusions . . . . . . . . . . . . . . . . . . . 125

6 Concluding Remarks and Future Directions $\quad 127$

6.1 Conclusion . . . . . . . . . . . . . . . . . . 127

6.2 Future Directions . . . . . . . . . . . . . . . . . . . . 128

6.2.1 Mobile Receiver Implementation and Characterization . . . . 128

6.2.2 Adaption for Color Rendering . . . . . . . . . . . . . . . . . . 130

$\begin{array}{ll}\text { A Designed Constellations } & 147\end{array}$

A.1 Constellations from Chapter $3 \ldots \ldots$. . . . . . . . . . 147

A.2 Constellations from Chapter $5 \ldots \ldots$. . . . . . . . 151

B Calculating Correlated Color Temperature 155 


\section{List of Figures}

1.1 Depiction of a lead vehicle beaming data to a following vehicle via LED tail lamps .............................. 8

2.1 Cross section of human eye and ganglion cell . . . . . . . . . . . . 14

2.2 Human Scotopic and Photopic luminosity functions . . . . . . . . . . 16

2.3 Indoor VLC example test room f . . . . . . . . . . . . . . . . . 17

2.4 Relative human brightness perception versus relative luminance . . . 19

2.5 CIE 1931, $X Y Z$ color matching functions . . . . . . . . . . . 21

2.6 CIE $1931 x y$ chromaticity chart. The shaded area represents colors that cannot be accurately reproduced by a typical computer monitor 23

2.7 IM/DD channel model . . . . . . . . . . . . . . . . . . . . 31

2.8 Blue LED, yellow phosphor based white LED cross section . . . . . . 33

2.9 RGB LED cross section . . . . . . . . . . . . . . . . . . . 34

2.10 Example of NRZ OOK (top) and Manchester OOK(bottom) . . . . . 43

2.11 Example of VPPM data pulses . . . . . . . . . . . . . . . 44

2.12 Example of metameric modulation in the $x y$ chromaticity space with two LED sets . . . . . . . . . . . . . . . . . . . . . . . 46

2.13 Functional block diagram of VLC channel that supports the CSK modulation format . . . . . . . . . . . . . . . . . . 47 
2.14 IEEE Standard 802.15.7 4-CSK design rule . . . . . . . . . . . . 50

2.15 IEEE Standard 802.15.7 8-CSK design rule . . . . . . . . . . . . . . 51

2.16 IEEE Standard 802.15.7 16-CSK design rule . . . . . . . . . . . . 52

3.1 Approximate minimum distance versus $\beta$ for three sets of two distances 63

3.2 8-CSK with and without specified color balance. $L=1, L_{r, g, b}>L$ and identity channel matrix. . . . . . . . . . . . . . . . . 70

3.3 32-CSK for two different color balance points. Both cases have identity channel matrix, with $L_{r, g, b} \geq L$. . . . . . . . . . . . . . 71

3.4 16-CSK with and without dimension truncation, including color balancing. Both cases have identity channel matrix . . . . . . . . . . . 72

3.5 Optimal 16-CSK alphabet, in both the normalized design space and transmitted intensity coordinates, for $L=1, L_{r, g, b} \geq L$, and non-ideal invertible channel matrix . . . . . . . . . . . . . . .

3.6 128-CSK, $L_{r, g, b} \geq L$ with no color balance for both the identity channel matrix and the channel matrix specified by equation (3.19) . . . . .

3.7 Minimum distance, in the normalized design space, versus constellation size for $L_{r}=L_{b}=L_{g} \geq L$, no color balance, and $\mathbf{H}$ is the identity $\operatorname{matrix} \ldots \ldots \ldots \ldots \ldots \ldots$

3.8 128-CSK, achieved with the lattice heuristic, for $L_{r, g, b} \geq L$ with no color balance for both the identity channel matrix and the channel matrix specified by equation $(3.19) \ldots \ldots \ldots \ldots$

3.9 Depiction of 3D 8-CSK constellation as seen by both the transmitter and receiver. Channel matrix is identity, no operating color is specified, $L_{\max }=1.1$ and $L_{\min }=0.9$. . . . . . . . . . . . . . 80 
3.10 Depiction of 3D 32-CSK constellation as seen by both the transmitter and receiver. Channel matrix is identity, $L_{\max }=1$ and $L_{\min }=0.1$, no independent peak constraints on a color channel, and with an operating chromaticity corresponding to $\mathbf{d}=[0.3443,0.4857,0.1700] \ldots$. . . . 81

3.11 Depiction of 3D 16-CSK constellation as seen by both the transmitter and receiver. Channel matrix be as in equation (3.19), $L_{\max }=1.1$ and $L_{\text {min }}=0.9$ and $\mathbf{d}=[0.2883,0.3203,0.3203] \ldots \ldots \ldots$. . . . 82

4.1 CSK experimental test bed . . . . . . . . . . . . 86

4.2 Functional block diagram of transmitter $\ldots \ldots \ldots \ldots$

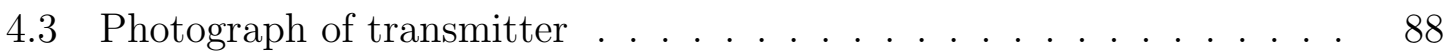

4.4 Linear variable current driver $\ldots \ldots \ldots$

4.5 Photograph of 3 -channel linear current driver $\ldots \ldots$. . . . . . . 89

4.6 Photograph of luminary housed inside transmitter test fixture . . . . 91

4.7 DC linearity of each LED used in the experiment $\ldots \ldots \ldots \ldots$

4.8 Functional block diagram of receiver $\ldots \ldots \ldots \ldots$

4.9 Responsivity of the PDA36A variable gain photo detector . . . . . . 94

4.10 Polyester filter transmission spectra . . . . . . . . . . . . 94

4.11 Measured magnitude, defined as $10 \log 10\left(v_{\text {peak-peak }}\right)$, versus frequency, in hertz, of a $200 \mathrm{~mA}$ peak to peak drive current, for the CSK channel at the input of the LED string (left) and output amplified photodiode (right). The photodiode bandwidth was measured separability for each colored LED . . . . . . . . . . . . . . . . . . . . . . . . 97

4.12 Normalized detector output versus drive current . . . . . . . . 98

4.13 Measured PDF and Quantile-Quantile plots of channel noise . . . . 99 
4.14 Orthogonal "Walsh" code . . . . . . . . . . . . . . . . . . . 100

4.15 Measured photodiode output versus illuminance . . . . . . . . . . . . 102

5.1 All possible transmit symbol (left), normalized design space with color temperature marked (right) . . . . . . . . . . . . . . 107

5.2 Simulated SER versus SNR, minimum distance, and depiction of IEEE 4-CSK, Optimized 4-CSK, and Optimized 3000 K 4-CSK . . . . . . . 111

5.3 Simulated SER versus SNR, minimum distance, and depiction of IEEE 8-CSK, Optimized 8-CSK, and Opted 3000 K 8-CSK . . . . . . . . . 112

5.4 Simulated SER versus SNR, minimum distance, and depiction of IEEE 16-CSK, Optimized 16-CSK, and Opted 3000 K 16-CSK . . . . . . 113

5.5 8-CSK optimized for a CCT of 2700K, 3000K, 3500K, 4000K, 4500K, $5000 \mathrm{~K}, 5700 \mathrm{~K}$ and $6500 \mathrm{~K} \ldots \ldots \ldots \ldots$

5.6 Simulated SER vs SNR for the 8-CSK constellations depicted in Figure

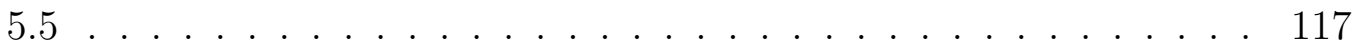

5.7 Unitless orthographic projection of received symbols transmitted through the experimental link for a fixed operating current of $200 \mathrm{~mA}$. The decision region centers are marked with a red cross. (a) Optimal 8-CSK (b) $3000 \mathrm{~K} 16-\mathrm{CSK} \ldots \ldots \ldots \ldots$

5.8 Experimental SER versus electrical SNR for 4-CSK . . . . . . . . . 121

5.9 Experimental SER versus electrical SNR for 8-CSK . . . . . . . . . . 122

5.10 Experimental SER versus electrical SNR for 16-CSK . . . . . . . . . 122

5.11 SER versus experimental Illuminance for various constellations . . . . 124

6.1 Simplified structure of a tri-chromatic ambient light sensor . . . . . . 129 


\section{Chapter 1}

\section{Introduction}

The United State of America (USA) Federal Communications Commission (FCC) has outlined a looming spectrum shortage in their National Broadband Plan that would make satisfying the increasing demand for mobile data services in urban city centers, with current resources and technology, impossible within the next ten years [1]. Cisco Systems Inc., and other industry participants, expect that mobile Internet services will account for $71 \%$ (7.6 Exabytes per month) of total mobile data traffic by 2016 , compared to 45\% (269 Petabytes per month) in 2011 [2, 3].

To address the rise in demand for the mobile Internet, service providers are increasingly looking to offload traffic to cabled Wi-Fi networks. However, Wi-Fi hubs tend to cover large areas, forcing limited Wi-Fi spectrum to be shared with many users in populated areas, including non mobile users. A popular technique to compliment $\mathrm{Wi}-\mathrm{Fi}$ is the use of femtocells, which are extremely short range radio frequency $(\mathrm{RF})$ cell base stations that cover a small area in order to provide mobile connectivity via fixed connections such as cable or DSL. While femtocells have shown some success in household deployment, they are limited by cost and the interference they generate 
with outdoor base stations, limiting their proliferation $[3,4,5]$.

Accounting for traffic offload onto fixed networks, the FCC notes that an additional $800 \mathrm{MHz}$ of RF spectrum would be required by 2015 to meet this growing demand in urban city centers; however, the availability of radio spectrum is scarce, as the majority of the usable RF spectrum has already been allocated [1]. Thus, the increasing demand for higher bandwidths and faster data transmission, coupled with the extremely limited availability of radio spectrum, have sparked an interest in new technologies to reduce the spectral load on RF mobile systems [6]. There are multiple research venues in the area of wireless networking to alleviate this problem:

1. Minimization of RF interference $[7,8]$

2. Improved reuse of the available frequency resources (e.g. cognitive networks) $[9,10]$

3. Utilization of unlicensed spectrum (e.g. $60 \mathrm{GHz}$ RF carriers and optical carriers) $[11,12,13]$

Specifically, the utilization of the visible light spectrum has recently received attention as a means to reduce the spectral load on radio systems. Communication using visible the light spectrum has been termed visible light communications (VLC) by the research community $[14,15]$. As visible light cannot penetrate opaque surfaces, VLC transceivers can fulfill a similar role to femtocells, aiding in the offload of mobile data onto a cabled connection without interfering with RF transceivers. The use of visible light for communications is motivated by recent developments in LED technology that are leading the way toward their full adoption as a replacement for 
incandescent and fluorescent lighting $[16,17]$. As LEDs are seeing a larger installation base in home and office settings, the deployment costs of VLC devices can be substantially reduced by simply integrating modulation hardware with the existing LED luminaries.

Light emitting diodes are attractive as VLC transmitters as they can be modulated to fulfill the primary role of illumination while simultaneously communicating data. The average life span of an LED is about 60 kilo-hours, compared to the average lifetime expectancy of incandescent bulbs at 1.2 kilo-hours, and fluorescent lamps at 10 kilo-hours [18]. Furthermore, LEDs are more energy efficient, consuming only $10 \%$ of the power used by incandescent bulbs, and $50 \%$ of that used by fluorescent lamps for the same levels of illumination [18]. While the installation cost of an LED light source is considerably more than that of an incandescent bulb, is has been shown that operating an LED source for 60 kilo-hours is $\$ 88$, compared to $\$ 783$ and $\$ 186$ for incandescent florescent sources respectively [16]. Additionally, the Canadian federal government and parts of the USA have proposed new energy efficiency standards which will prevent incandescent light bulbs from being imported into their respective regions by 2014 [19]. These standards have motivated the adoption of sustainable LED lighting into both new and old infrastructure.

\subsection{State of Visible Light Communications}

Visible light communications was pioneered in Japan, leading to the creation of the Visible Light Communications Consortium (VLCC) [20]. The mandate of VLCC is to research and develop VLC technologies in Japan that will lead to the standardization of VLC practice. Interest in VLC continued with project "hOME Gigabit Access" 
(OMEGA) in Europe, which was intended to deliver a 1 Gigabit/second (Gbps) data rate via interior lighting, with further VLC investigations being conducted worldwide $[14,15,21,22,23]$. Since the completion of project OMEGA in 2011, the IEEE ratified a new VLC standard, 802.15.7, in 2012 as part of the Wireless Personal Area Networks Standards [24].

Specifically, 802.15.7 defines the Physical (PHY) and Media Access Control (MAC) layers for short-range optical wireless communications using visible light, and considers the impact of modulation on illumination. The PHY and MAC specifications are capable of delivering data rates from $11 \mathrm{kbps}$ to $96 \mathrm{Mbps}$; the later being sufficient to support audio and video multimedia services. The standard also considers the mobility of the visible link, compatibility with visible-light infrastructures, impairments due to noise and interference from sources such as ambient light. Furthermore, IEEE 802.15.7 adheres to all applicable eye safety regulations outlined by the governing standardization bodies and directly references IEEE PAR1789, which outlines recommended practices for modulating current in high brightness LEDs in order to mitigate potential negative effects on human health [25]. In addition to 802.15.7, there are several Japanese standards preceding IEEE 802.15.7, with the Japan Electronics and Information Technology Industries Association (JEITA) being the most prominent $[26,27]$.

\subsubsection{Visible Light Communications Challenges}

The primary challenge in VLC is that the modulation bandwidth of the white light phosphor LEDs contained in the majority of commercial LED light fixtures is limited 
to approximately $2 \mathrm{MHz}$, making giga bit per second (Gbps) communication links currently unachievable with the typical light power incident on most household surfaces [28]. A common method to increase the modulation bandwidth of LED luminaries is to apply an optical filter at the receiver. White light LEDs typically produce light by using a highly efficient blue LED to excite a yellow phosphor coating that reduces the bandwidth of the LED. By detecting only the blue component of these phosphor LEDs, as was demonstrated in project OMEGA, the modulation bandwidth of the VLC link can be increased to approximately $12 \mathrm{MHz}[14]$.

Aside from increasing the modulation bandwidth, bandwidth efficient modulation schemes, such as pulse amplitude modulation (PAM) or orthogonal frequency division multiplexing (OFDM), are an effective means of increasing data throughput. This is particularly important to VLC as it has been shown that SNR in a VLC channel is typically in excess of $60 \mathrm{~dB}$ electrical, allowing the receiver to easily detect multiple amplitude levels in a signal [12]. For the same signal power and bit error rate, the work of [29] shows a data rate of 200 Mbps using unipolar OFDM while [30] achieved 125 Mbps using a binary signaling scheme; both techniques reported the use of blue light optical filtering at the receiver.

In addition to increasing bandwidth efficiency, the visible light spectrum can be used more efficiently by producing white light with the use of red-green-blue (RGB) LEDs. As each color can be modulated independently, it is possible to achieve a threefold increase in data throughput through the use of wavelength division multiplexing (WDM), where data are sent on each wavelength independently. However, there is concern that independent modulation of each color may create undesirable light sources that vary in color or intensity over time [24,31]. To help combat these 
concerns, two modulation schemes, termed color-shift keying (CSK) and metameric modulation (MM), have been proposed, with the specific intention of modulating multiple colored LEDs to produce adequate illumination. While new research suggests that RGB LEDs can achieve efficiencies equal to those of white light phosphor LEDs, the primary drawback of RGB LEDs is that their efficiency is currently half that of phosphor LEDs [32, 33, 34]. Therefore, the primary focus of the lighting industries is on the production and proliferation of white light phosphor LEDs, with RGB LEDs accommodating niche lighting applications [35, 36, 37].

Another significant challenge with VLC is providing a reliable up-link, as VLC channels are naturally broadcast (down-link) channels. VLC also requires the presence of light, which leads to the problem of finding effective methods to communicate when the light source needs to appear to be off. Furthermore, for VLC to be successful, it must integrate with radio networks to form seamless cooperative networks in order to achieve the goal of relieving radio frequency congestions [38]. Lastly, with VLC depending heavily on the explosive growth of the solid state lighting market, VLC systems would benefit from successful integration with both Power-over-Ethernet (PoE) and Power-Line-Communications (PLC) technologies to limit implementation costs and improve commercial viability $[39,40]$.

\subsubsection{Visible Light Communication Applications}

While VLC can be used to generate personal area networks, it also has a host of other applications, including tracking, location based services, vehicle to vehicle communication, and the ability to be used in areas where RF is not permitted. 


\section{Location Based Services and Tracking}

ByteLight, a venture backed startup out of Cambridge Massachusetts, has patented an indoor location system using LED lighting [15]. Through the exploitation of the directionality of visible light, the technology is capable of providing a user his/her location, and also tracks user traffic throughout a space. Therefore, VLC systems in general are able to provide location specific services or advertising to users. Examples of this include targeted marketing materials, such as coupons, or directions to supporting vendors. Additionally there is work on true indoor GPS systems (providing location and trajectory) being conducted at Boston University, McMaster University and the Geospatial Information Authority in Japan [41, 42, 43].

\section{Personal Area Networks}

Project OMEGA was a 36 month project, started in 2008, with the goal of developing a user-friendly home access network capable of delivering high-bandwidth services and content at a data rate of 1 Gbps. The project successfully achieved 1 Gbps using the infrared band, and was able to demonstrate a fully functioning VLC communication system with a down link speed of $100 \mathrm{Mbps}$. The link demonstrated consisted of 16 high-power LED lamps that were mounted into the ceiling and configured to broadcast four high definition videos streams continuously. This was accomplished by aggregating the output of four video players onto an Ethernet data stream, and by modulating this data onto the electrical driving current of the LED lamps. 

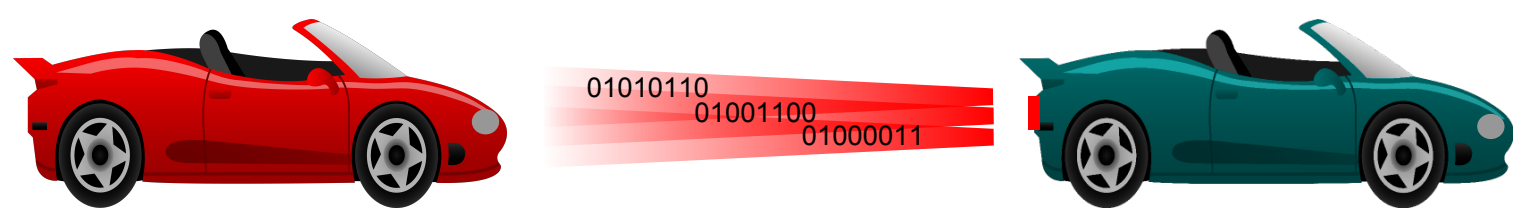

Figure 1.1: Depiction of a lead vehicle beaming data to a following vehicle via LED tail lamps

\section{Vehicle to Vehicle, and Vehicle to Infrastructure Communication}

Figure 1.1 depicts an example of two vehicles communicating via their headlights to warn the rear vehicle of abrupt stops. In 2008, the R8 super-car from Audi became the first commercial vehicle with full LED headlamps, followed by the Lexus LS [44]. With the adoption of LEDs in automotive lighting, vehicles can potentially use VLC to share data with each other. Furthermore, traffic lights are making the transition to LEDs, providing an opportunity for a VLC channel to exist between city infrastructure and a vehicle $[45,46]$.

\section{Areas Un-serviceable by Radio Frequency}

Hospitals and environments requiring intrinsically safe communication, such as petrochemical production facilities, are examples where radio frequency communications are not permitted. Furthermore, there is an ongoing project in Japan that is intended to incorporate VLC applications into hospitals and health care institutions [20].

\subsection{Thesis Contributions}

This work considers the design and implementation of an RGB LED VLC communication link using the CSK modulation format. While VLC claims access to Tera-Hertz of unlicensed spectrum, interest in VLC primarily lies with the use of white light 
phosphor LEDs, which modulate the same data over the entire visible spectrum. Therefore, white light LEDs inefficiently utilize the optical spectrum, thus limiting the achievable data throughput of the system. Spectral efficiency can be improved through the use of RGB LED's, as they partition the visible light spectrum into three separate communication channels, allowing for greater data throughput. Indeed phosphor white LEDs are the most prevalent on the market due to their higher efficiency. However, RGB LED's luminaries still exist on the market, such as the Philips Hue product, as they are capable of providing additional features, such as color adaption to ambient light [37]. Furthermore, Section 1.1.1 mentioned that there has been success in improving the efficiency of green LED's, which could pave the way for efficient RGB LED lighting.

The first contribution of this work is a rigorous framework for the design and optimization of CSK symbol sets of any size using deterministic convex optimization techniques. Unlike the current standardized design rules outlined in IEEE 802.15.7 Section 12.2, this work optimizes constellations against cross talk between each color channel. Additionally, the method accounts for the human color perception of each alphabet, based on the study of colorimetry, allowing the source color to be easily adjusted in order to meet lighting industry color standards. The design method is subsequently adapted to allow for a specified ripple in the luminous flux to produce three dimensional CSK constellations with improved communications qualities.

As of writing, only two other works exist on the design of CSK constellations. The first considered the optimization of CSK constellations, based on statistical sphere packing techniques, that allowed an alphabet mean to be specified, while accounting for channel cross talk [47]. However, this work did relate the notion of the alphabet 
mean to the established field of colorimetry. The second work reviewed binary CSK constellations from colorimetry principles and optimized error performance through non uniform signaling [48]. However, this work only considered binary constellations and did not establish a link between the intensities of CSK symbols needed to generate a specific operating color, nor did it consider the cross talk between the three color channels. Furthermore, neither of these past works examined the relaxation of the CSK constraints to allow ripple in the luminous flux of the luminary.

The second contribution of this work is an efficient heuristic for designing large constellations. When no average perceived color is specified and the symbol set is large, the optimized constellations all exhibit near hexagonal packing. It is suggested that a hexagonal lattice intersected with the constraint region will provide adequate symbol sets. The hexagonal symbol sets may be more desirable to system designers, as regularly spaced symbols require simpler decoding procedures than the arbitrary constellation typically designed using the convex optimization approach.

The final contribution of this work is the implementation of a CSK transmitter. An RGB LED luminary is constructed and a CSK communication channel is characterized with use of an instrumentation photodetector and an oscilloscope. The implemented test bed is used to experimentally measure the error performance of each constellation against SNR. Additionally, the test bed allows measurement of the available SNR against illumination allowing the comparison of CSK error performance against the lighting industries illumination requirements. 


\subsection{Outline of Thesis}

The remainder of this thesis is organized into four chapters. Chapter 2 provides an overview of the human perception of visible light intensity and color. Industry lighting standards on intensity and color are reviewed and the VLC channel is modeled with the constraints that each standard imposes on VLC. Additionally, the strengths and weakness of phosphor and RGB LEDs for VLC are discussed. Chapter 2 also derives the link between lighting industry illumination requirements and the Signal to Noise (SNR) ratio available in VLC. This is followed by a review of standardized and unstandardized VLC compatible modulation schemes. The chapter concludes with a detailed review of CSK and the CSK VLC channel model, including CSK specific constraints as well as the current CSK constellation design techniques and their limitations.

Chapter 3 provides a rigorous framework, based on convex optimization, for the design of CSK constellations. The design problem is formulated such that it is compatible with interior point optimization techniques. Furthermore, the framework allows constellations to be designed to operate with constant electrical current or intensity while maintaining a specified perceived color. An advantage of this approach is that it is able to optimize constellations based on the crosstalk between the multiple color channels required for CSK and is able to produce symbol set for any size constellation. Chapter 3 also provides a heuristic for designing large regular constellations, and concludes with techniques for designing three dimensional CSK constellations.

Chapter 4 discusses the implementation and characterization of a CSK compatible VLC link. To the knowledge of the author, this is the first CSK compatible VLC link presented in literature. Each component used in the system is specified, 
relevant figures of merit are determined, and the available SNR versus illumination is measured.

Chapter 5 discusses the use of the methods discussed in Chapter 3 to optimize constellations based on the channel characterized in Chapter 4. Constellations are also design based on the existing standardized CSK design rules to act as a performance benchmark. The error performance of each constellation is then simulated and measured over different illumination levels. Comparisons between the proposed optimization method and the IEEE design rules are then drawn. The chapter concludes with alphabet selection guidelines based on required illumination. 


\section{Chapter 2}

\section{Human Vision, Lighting Standards and Channel Modeling}

This chapter provides an overview of human vision, lighting standards and system hardware components, as well as the constraints they impose on VLC. This chapter reviews the general VLC channel model, provides an example of the available SNR in a typical VLC scenario, and reviews both standardized and unstandardized VLC compatible modulation schemes. Lastly, the CSK channel model is derived, including CSK specific constraints, as well as the current limitations in the design of CSK symbol sets.

\subsection{Human Perception of Light}

Figure 2.1 depicts the cross section of the human eye and a simplified component diagram of the retina. Light incident on the cornea passes through the pupil, whose aperture is adjusted by the iris, and is focused by the lens onto the retina. The 

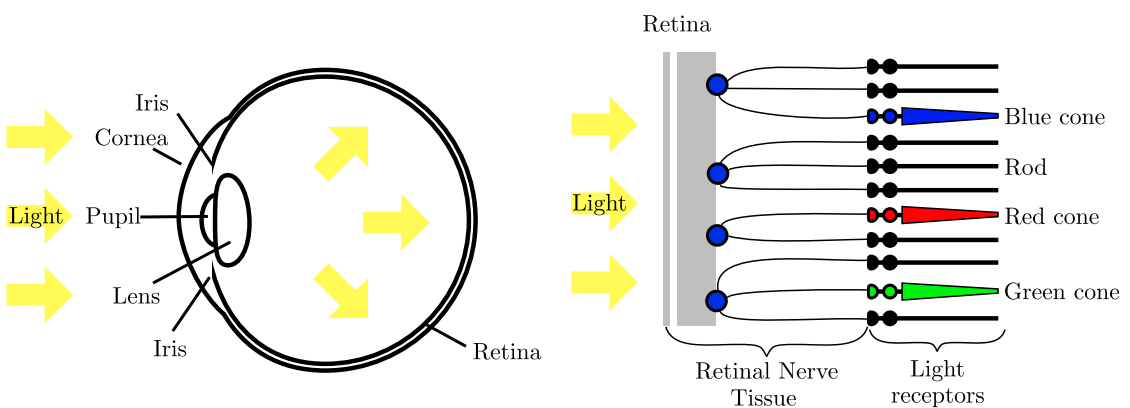

Figure 2.1: Human eye cross section (left), retinal ganglion cell (right) adapted and modified from [51]

perception of light intensity and color is mediated by the rod and cone receptor cells found in the retina [49, p. 85]. Rods are highly sensitive to light and provide monochrome vision in poor light, while cones are used for both the perception of color and for the detection of fine detail. The human eye is able to adapt its vision to one of three vision regimes, based on ambient light levels [50]. Photopic vision occurs at high ambient light levels, such as during the day, and is mediated by the cone receptors. Scotopic vision occurs at low ambient light levels, and is mediated by rod receptors. While rods have a much higher sensitivity to light than the cones, the perception of color is lost in the Scotopic vision regime. Mesopic vision occurs in between Photopic and Scotopic. During the Mesopic regime, humans retain color perception, but with less acuity than in Photopic vision.

\subsubsection{Perception of Intensity}

The intensity of light can be quantified with either radiometric or photometric quantities. Radiometric quantities measure light intensity in terms of the sources absolute power in space while photometric quantities account the interaction of light with the human eye. The base unit of radiometric quantities is known as flux. Flux is the 
total optical power, in Watts, the source radiates and is expressed by,

$$
\Phi_{R}=\int P(\lambda) \mathrm{d} \lambda
$$

where $P(\lambda)$ is the source spectral power density (SPD), which represents the amount of power of the source, in watts, present at each wavelength in the electromagnetic spectrum. Radiant intensity is defined as radiant flux per unit solid angle,

$$
I_{R}(\theta)=\frac{\mathrm{d} \Phi_{R}}{\mathrm{~d} \Omega}
$$

Figure 2.2 depicts the normalized CIE luminosity functions which describe the normalized sensitivity of the human eye to different wavelengths of light, depending on the active vision regime [49, p. 143] [52]. While radiometric flux and intensity are useful for determining the power response in electronics, it is clear from Figure 2.2 that the human eye is not equally sensitive to all wavelengths of light and that radiometric flux does not represent how humans perceive light. Photometric quantities were created in order to account for these sensitivity curves. The base unit of photometric quantities is luminous flux with unit lumen; it is a measure of the useful light power of a source and is stated on luminary data sheets. Luminous flux is derived by weighting the SPD of a light source at each wavelength by a luminosity function,

$$
\Phi_{L}=K_{w} \int_{\lambda_{380}}^{\lambda_{780}} P(\lambda) L(\lambda) \mathrm{d} \lambda
$$

where $L(\lambda)$ represents the normalized luminosity function and $K_{w}$, the scaling factor, is known as the maximum luminous efficacy with units lumen per watt. In practice, 


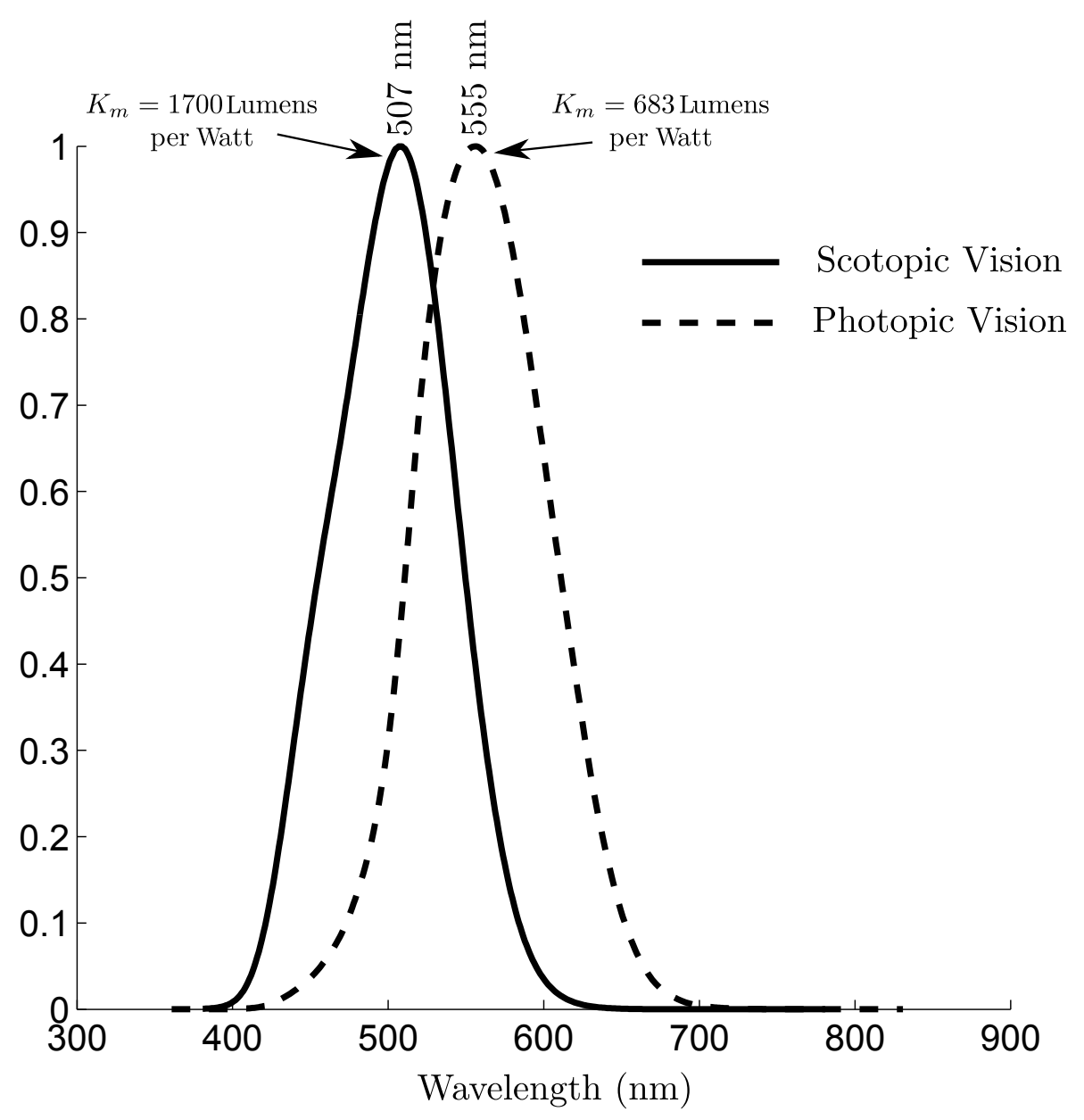

Figure 2.2: Human Scotopic and Photopic luminosity functions constructed from data tables provided by [52] 


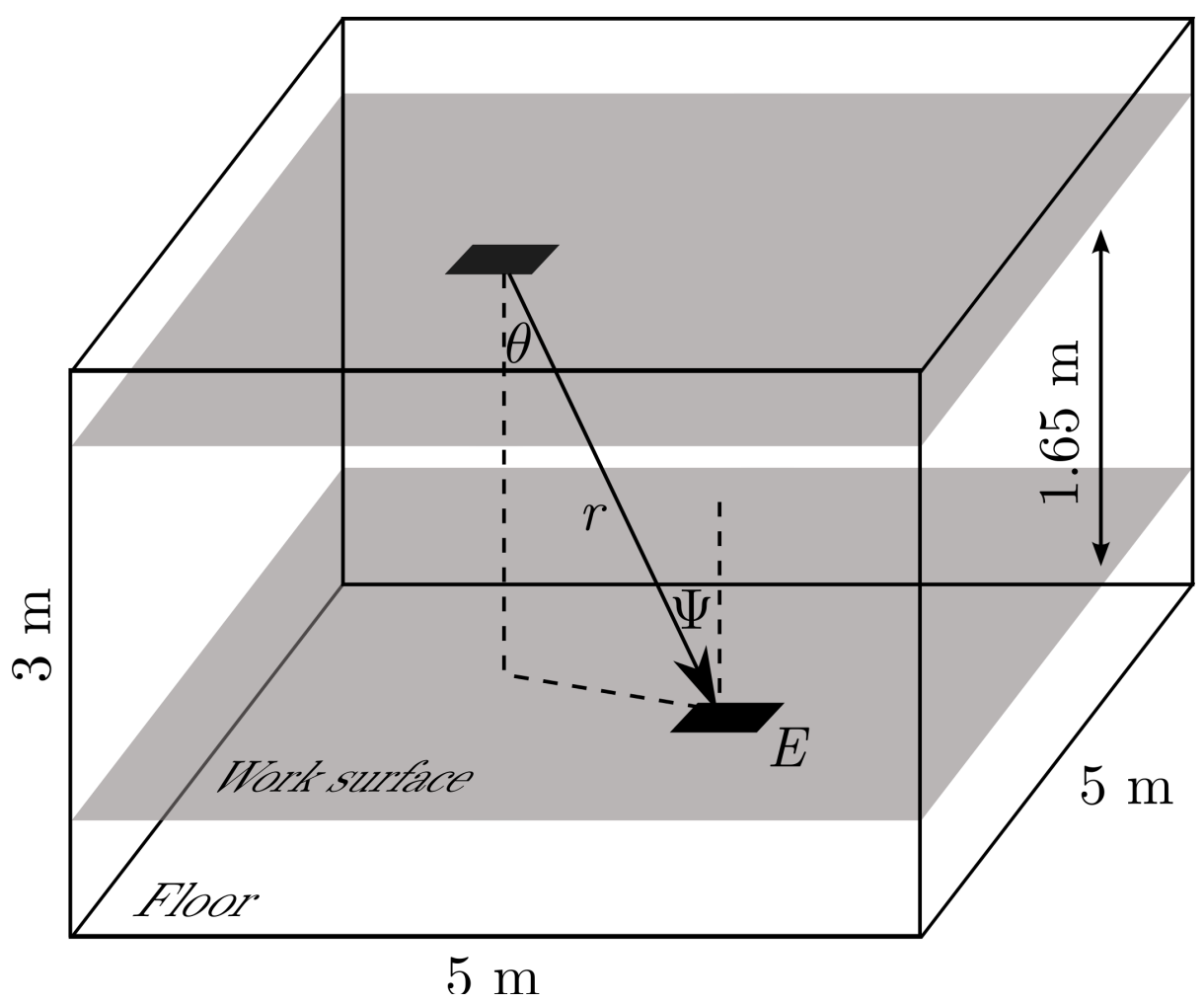

Figure 2.3: Indoor VLC example test room [28]

photometry is based solely on the Photopic luminosity function, even if light levels suggest a Scotopic or Mesopic regime.

While luminous flux describes the total useful light power of a source, it does not indicate how well lit a surface illuminated by the source is. The photometric quantity used to quantify the useful light power that falls onto the surface of interest is referred to as illuminance. Figure 2.3 provides an example of a room being illuminated by an LED luminary. The illuminance, $E$, is defined as the luminous flux per unit area, and is expressed by,

$$
E=\frac{\mathrm{d} \Phi_{L}}{\mathrm{~d} A}=\frac{1}{r^{2}} \frac{\mathrm{d} \Phi_{L}}{\mathrm{~d} \Omega}=\frac{I_{L}(\theta)}{r^{2}}
$$

where $\Omega$ is the solid angle, $r$ is the distance to the illuminated surface, and $I_{L}(\theta)=$ 
$\mathrm{d} \Phi_{L} / \mathrm{d} \Omega$ is defined as the luminous intensity of the source [28]. For light sources with Lambertian radiation patterns, as is the case with LEDs, $I_{L}(\theta)$ is represented by,

$$
I_{L}(\theta)=I_{0} \cos ^{m}(\theta)
$$

where $m$ is the Lambertian index and $I_{0}=I_{L}(\theta=0)=(m+1) \Phi_{L} /(2 \pi)$ is the maximum luminous intensity of the source. Additionally, the Lambertian index, $m$, is a function of the LEDs apex angle; the angle between the axis where the LED gives its highest luminous intensity and the axis where that intensity is reduced by one half of its maximum,

$$
m=-\frac{1}{\log _{2}\left(\cos \left(\theta_{1 / 2}\right)\right.}
$$

Based on manufacturer data sheets, the majority of currently produced LED luminaries have an apex angle of approximately 60 degrees, resulting in $m=1[35,36,53,54]$. Furthermore, the angle of incidence $(\Psi)$ affects illuminance of flat horizontal surfaces, with the horizontal illumination defined as,

$$
E_{h}=E \cos \Psi=I_{0} \frac{\cos ^{m}(\theta) \cos (\Psi)}{r^{2}}
$$

While photometry accounts for the wavelength dependence of the human eye, it does not model the perceived brightness of different photometric intensities by a standard human observer $[49$, p. 152$][50,55]$. Figure 2.4 shows the average relative human perception of brightness against the relative illuminance incident on the eye. The result depicted Figure 2.4 occurs primarily because the human eye will adjust its aperture via its iris, based on the total illuminance incident upon it, effectively reducing the power incident on the rod and cone cells. The iris is more likely to reject 


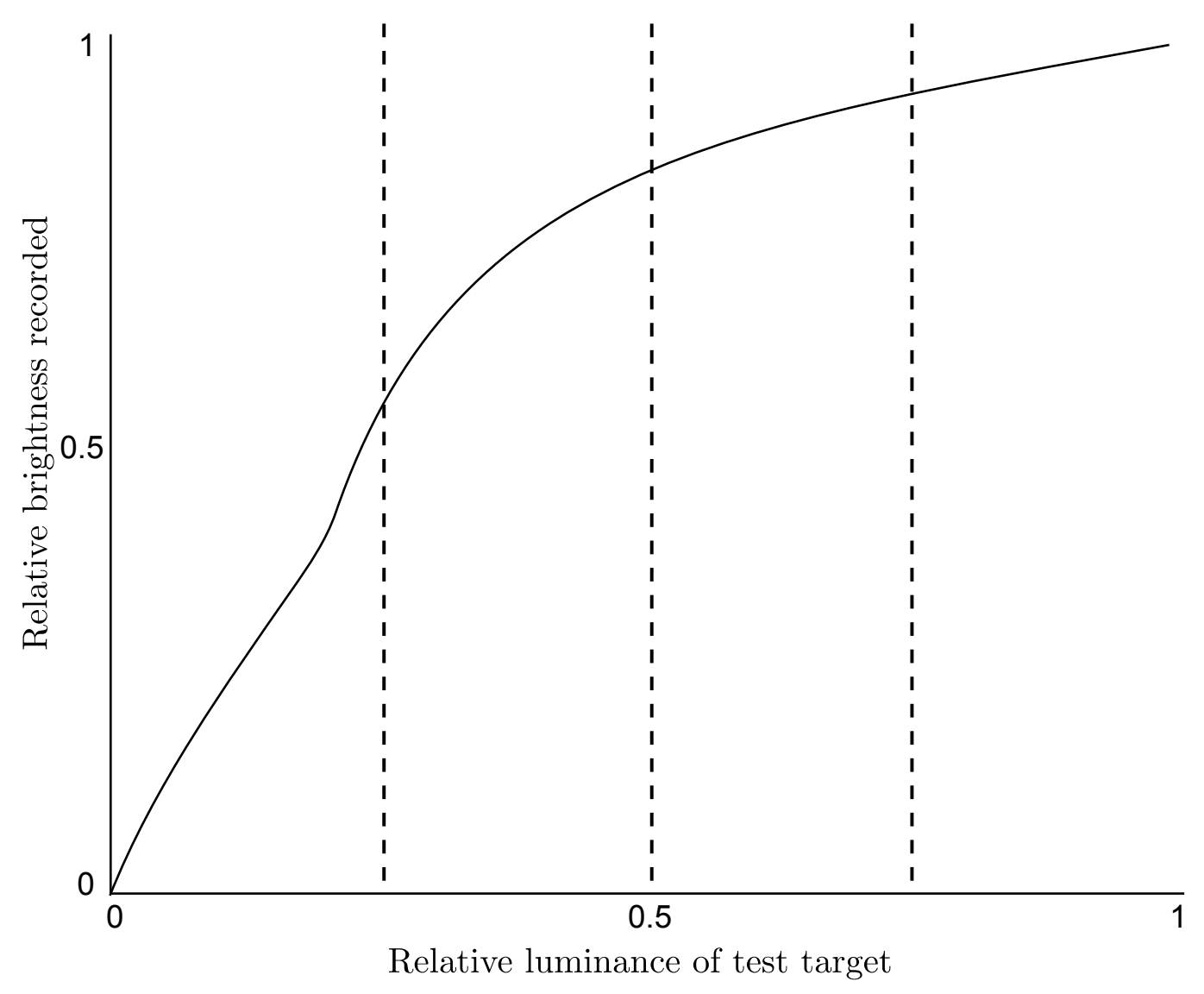

Figure 2.4: Relative human brightness perception versus relative luminance adapted from data presented in $[50]$

higher intensities to avoid saturation of, and damage to, the retina thus leading to the non linear relationship seen in Figure 2.4 [50]. It is important to note that since the muscles in the eye take time to react and adjust the iris, the perception of brightness is different for a flash of light compared a light source that appears constant. In the case of a flash, the human perception of brightness is nearly linear with light intensity $[55]$. 


\subsubsection{Perception of Color}

There are three types of cone receptors present in the human eye: short, medium, and long. Short cones correspond to the human perception of blue light, medium cones correspond to green light, and long cones correspond to red light [49, p. 275]. The study of quantifying the human perception of color is referred to as colorimetry. The basis of colorimetry is the mapping of a range of physically produced colors to the sensations registered by the three types of cone receptors. The most widely used mapping is the CIE $1931 X Y Z$ color space that was proposed by the International Commission on Illumination (CIE) in 1931 [52]. The CIE XYZ 1931 color space is capable of representing all possible colors that the standard human eye is capable of perceiving, and was derived through a human study [52]. The color space is defined by a set of three unitless primary colors, known as the $X Y Z$ tristimulus values. All colors perceived by the human eye are an additive mixture (linear combination) of the $X Y Z$ tristimulus, with $X Y Z$ defined by,

$$
[X, Y, Z]=\int_{\lambda_{380}}^{\lambda_{780}} P(\lambda)[x(\lambda), y(\lambda), z(\lambda)] \mathrm{d} \lambda
$$

where $x(\lambda), y(\lambda)$ and $z(\lambda)$ are known as the $X Y Z$ color matching functions, which can be seen in Figure 2.5. The color matching functions are used to relate the sensitivity of the human eye to each tristimulus primary and are collectively known as the CIE two degree standard observer [52].

While the $X Y Z$ color space is three dimensional, the concept of color can be broken down into two components; relative luminous flux and chromaticity. For example, under the $X Y Z$ model, white and gray are considered to be the same 


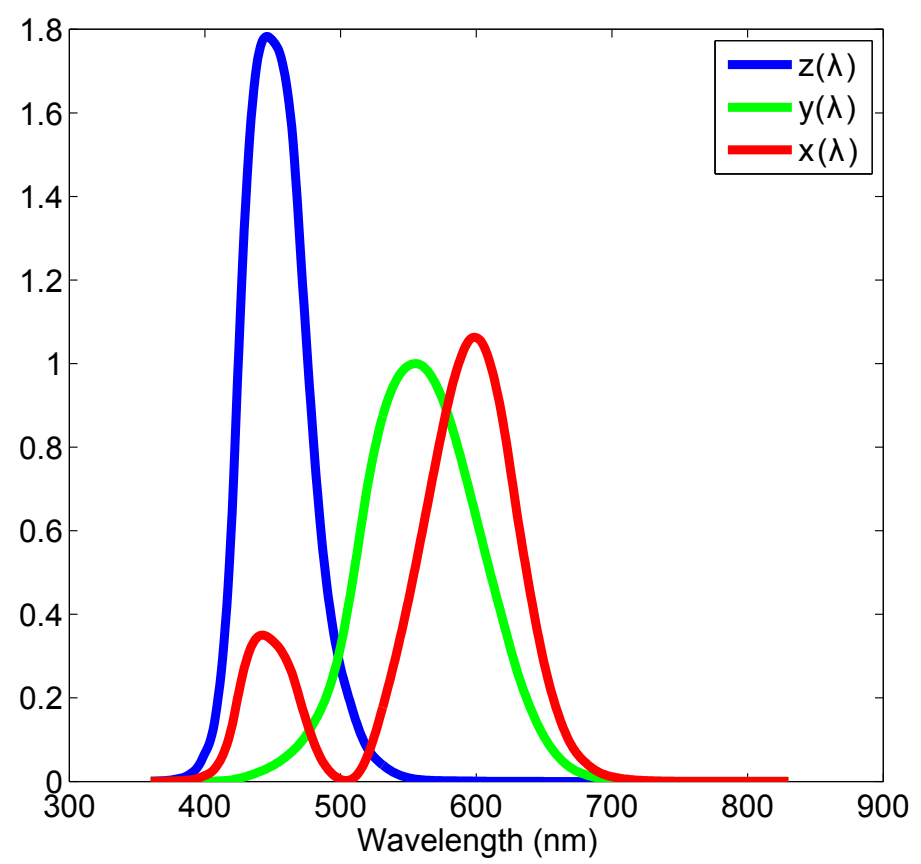

Figure 2.5: CIE 1931, $X Y Z$ color matching functions [52]

color, with white having a higher relative luminous flux than gray. This motivation led to the design of the $Y$ primary color matching function to be the same as the Photopic luminosity function [52]. Therefore $Y$ represents the relative luminous flux of the produced color, with the actual luminous flux found by multiplying $Y$ by the maximum luminous efficacy defined in section 2.1.1. In order to define a color metric independent of luminous flux, $Y$, the $X$ and $Z$ tristimulus values are normalized by the sum of $X Y Z$ to produce the two dimensional unitless color metric referred to as chromaticity,

$$
[x, y]=\left[\frac{X}{X+Y+Z}, \frac{Y}{X+Y+Z}\right]
$$

where $x$ and $y$ represent the perceived color the corresponding $X Y Z$ tristimulus. Figure 2.6 depicts the CIE $x y$ chromaticity diagram, formed by the intersection of the 
plane defined by $X+Y+Z=1$ with the $X Y Z$ color space. The chromaticity diagram represents all of the colors visible to the average human observer, and is referred to as the gamut of human vision. The outer curved boundary, excluding the straight line at the bottom, is known as the spectral locus and represents monochromatic light with wavelengths shown in nanometers. Given an $x y$ chromaticity and a desired relative luminous flux, $Y$, the $X$ and $Z$ tristimulus values can be retrieved,

$$
[X, Z]=\left[\frac{Y}{y} x, \frac{Y}{y}(1-x-y)\right]
$$

\section{Color Mixing}

Recall from (2.8) that the $X Y Z$ tristimulus are defined by the integrations of the source SPD with a set of weighting functions. As this is a linear process, new colors can be constructed by taking non-negative linear combinations of the $X Y Z$ values of two or more colors; non negativity is required as it is physically impossible to generate negative luminous flux, as defined by the $Y$ tristimulus. Therefore, considering three colored LEDs, and assuming that the SPD of each scales linearly over the allowable range of driving currents, the relative proportions of luminous flux, of the RGB LEDs, required to produce the desired chromaticity on average, can be calculated by,

$$
\left[\begin{array}{lll}
\frac{X_{r}}{Y_{r}} & \frac{X_{g}}{Y_{g}} & \frac{X_{b}}{Y_{b}} \\
\frac{Y_{r}}{Y_{r}} & \frac{Y_{g}}{Y_{g}} & \frac{Y_{b}}{Y_{b}} \\
\frac{Z_{r}}{Y_{r}} & \frac{Z_{g}}{Y_{g}} & \frac{Z_{b}}{Y_{b}}
\end{array}\right] \mathbf{d}=\left[\begin{array}{c}
\frac{X_{d}}{Y_{d}} \\
\frac{Y_{d}}{Y_{d}} \\
\frac{Z_{d}}{Y_{d}}
\end{array}\right]
$$

where the elements of $\mathbf{d}$ represent the contributing of each colored LEDs average luminous flux to the total average luminous flux, $X Y Z_{d}$ represent the desired $X Y Z$ 


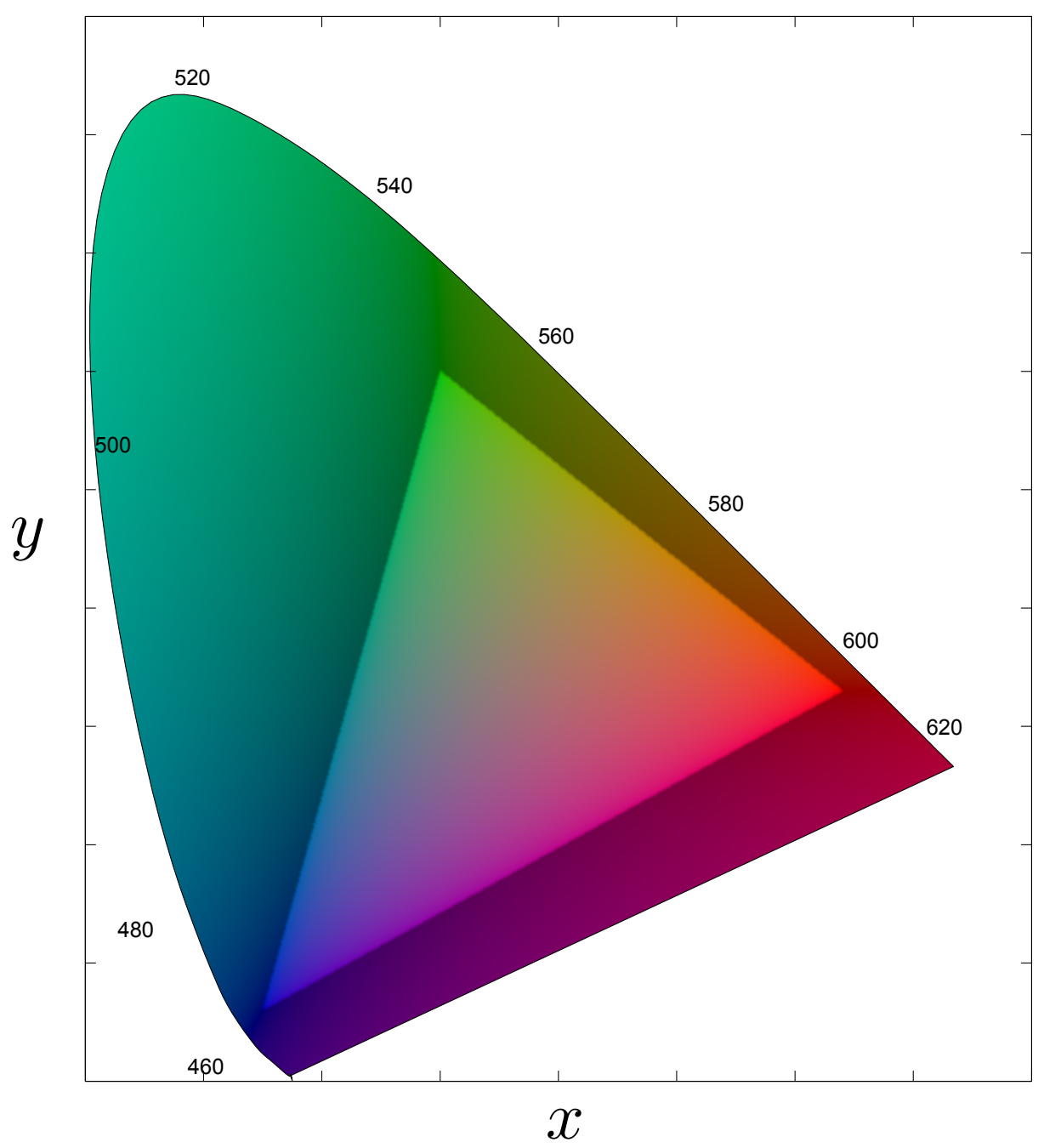

Figure 2.6: CIE $1931 x y$ chromaticity chart. The shaded area represents colors that cannot be accurately reproduced by a typical computer monitor 
tristimulus of the RGB combination, and $X Y Z_{r, g, b}$ represent the $X Y Z$ tristimulus values of the red, green and blue LEDs respectively. With $\mathbf{d}$ representing the relative contributions of each LED to the total luminous flux, the desired output luminous flux, while maintaining the same color, is achieved by proportionally scaling the output luminous flux of each LED.

Interestingly, as the $x y$ chromaticity space is the intersection of a plane with the $X Y Z$ space, it follows that the gamut of colors produced by mixing three colored light sources must be convex, and is the triangle formed $x y$ chromaticity of each light source. Therefore, three real color sources, such as an RGB LED, cannot reproduce the entire gamut of human vision.

\subsubsection{Perception of Flicker}

Flicker refers to repeated fluctuations in a light sources intensity (luminous flux) or color (chromaticity) that are perceptible to the human observer [56, 57]. Flicker is perceived only when an intensity or color fluctuation lasts longer than the physiological response of the rod and cone cells observing the stimulus [58] [49, p. 104]. The most widely used metrics for discerning whether or not a human will perceive intensity flicker and color flicker, when observing a light source, are known as critical flicker fusion threshold (CFF) and critical color fusion threshold (CCF) respectively $[57,58,59]$. CFF and CCF are defined as the frequency, in hertz, at which a light stimulus, with fluctuating intensity or color, appears to be completely steady to the average human observer[58]. Both CFF and CCF threshold are dependent on the average luminous flux, SPD, modulation frequency and modulation format of the source as well as the current vision regime $[50,58]$. Considering an incandescent light source 
with on-off modulation, CFF threshold saturates at about $15 \mathrm{~Hz}$ for rod-mediated (Scotopic) vision, whereas cone mediated (Photopic) vision reaches about $60 \mathrm{~Hz}$ before saturating [58]. The CCF is only measured during cone mediated vision, with the CCF of the average human observer plateauing at approximately $25 \mathrm{~Hz}$ for light sources that fluctuate between two colors of equal intensity [58].

Indeed, human perception of light plays a strong roll in the standardization and designing both luminaries and in VLC. Section 2.2 outlines both lighting and VLC standards, and the constraints they place on VLC.

\subsection{Standards and Constraints}

VLC systems must simultaneously provide proper illumination while transmitting data. This implies that the illumination standards used in the lighting industry must not be compromised in order to improve communication. Ultimately, restrictions are imposed on the degree to which the light source can flicker $[24,25,60]$, the average illuminance it must deliver [61, 62], the color it must produce [63], the color rendering ability of the source [64] and the peak intensity it can achieve[65, 66].

\section{Color Temperature}

Color temperature refers to the chromaticity of a black body radiator, such as a tungsten filament, that when heated, emits light with a chromaticity dependent on its temperature. Correlated color temperature (CCT), a lighting industry metric used to quantify the color, or whiteness, of a luminary, is defined as the temperature, in (K)elvin, of the black body radiator whose emitted chromaticity most closely represents the chromaticity of the luminary $[52,52,63]$. Therefore, it is possible for more 
than one chromaticity to map to the same CCT. The CCT can range from $1666.6 \mathrm{~K}$ to infinity; however, standards, such as Energy Star, which all luminaries must pass to be sold in North America, require the CCT be one of 2700K, 3000K, 3500K, 4000K, $4500 \mathrm{~K}, 5000 \mathrm{~K}, 5700 \mathrm{~K}$ or $6500 \mathrm{~K}$ [63]. Correlated color temperatures between $2700 \mathrm{~K}$ and $4500 \mathrm{~K}$ represent red and orange hues while $5000 \mathrm{~K}$ through $6500 \mathrm{~K}$ represent white and blue hues. The CIE has provided an excel spreadsheet that calculates the CCT of a given SPD [67]. The method used by the macros is summarized in the Appendix B.

\section{Color Rendering}

Color rendering, as outlined by the CIE, is the effect of an illuminant on the color appearance of objects in comparison to their color appearance under a reference illuminant $[51,63]$. The color rendering index (CRI) is the standard light industry metric for color rendering and is a unit-less quantitative metric of the color rendering ability of a broad spectrum light source [51, 52, 63, 64]. A high CRI (100 being perfect) implies that the source faithfully reproduces the correct color of objects that it illuminates, and is desired in everyday lighting. Standards, such as EN12464, specify that CRI should exceed 80 for areas where humans will perform prolonged tasks $[63,64]$.

It is important to note that CIE designed CRI with incandescent luminaries in mind, and it has received notable scrutiny as a useful metric for quantifying the color rendering ability or narrow-band light sources such as LEDs $[52,68,69]$. In fact, examples of LEDs luminaries have shown poor CRI, under 25, while apparently providing better color rendering than some florescent sources with CRI values of 80 
or more [70]. No standardized color rendering metric exists for narrow-band sources, but current investigations, including the new Color Quality Scale metric, are under study by the CIE $[52,69]$.

\section{Flicker}

The lighting industry has many standards, such as IEC 61000, that specify tolerances on the flickering of luminaries, as well as restrictions on power line flicker [56, 60]. Furthermore, the IEEE 802.15.7 standard states that it adheres to all lighting flicker standards, but makes no recommendation on allowable flicker levels. However, standard 802.15.7 does directly reference the PAR1789 standard, which outlines the biological effects of flickering stimulus. The document concludes that emission with no spectral components in the DC-200Hz band is unlikely to have serious health implications [25]. Therefore, care should be taken when designing visible light communication systems to limit the spectral power in the DC- $200 \mathrm{~Hz}$ band in order to limit human health risks.

\section{Illuminance}

The illuminance, as defined in (2.4), of light sources is governed by various standardization bodies including the International Organization for Standardization (ISO), the European Committee for Standardization (CEN) and Deutsches Institut für Normung (DIN). Table 2.1 provides typical illuminance values for different applications based in the DIN 5035 standard [62]. It can be seen that luminaries in the office require $500 \mathrm{~lx}$ on all work surfaces, while typical household rooms require an illuminance of $200 \mathrm{~lx}$. As seen from (2.4) and (2.3), illuminance is proportional to the SPD of the 
Table 2.1: Standardized illuminance based on activity pulled from DIN 5035 [62]

\begin{tabular}{|l|l|}
\hline \hline Activity & $\begin{array}{l}\text { Illumination } \\
\text { (Lux) }\end{array}$ \\
\hline Public areas with dark surroundings & $20-50$ \\
\hline Simple orientation for short visits & $50-100$ \\
\hline $\begin{array}{l}\text { Working areas where visual tasks are only occasionally } \\
\text { performed }\end{array}$ & $100-150$ \\
\hline Warehouses, Homes, Theaters, Archives & 150 \\
\hline Easy office work, Class rooms & 250 \\
\hline $\begin{array}{l}\text { Normal office work, PC work, Study library, Grocery } \\
\text { stores, Show rooms, Laboratories }\end{array}$ & 500 \\
\hline $\begin{array}{l}\text { Supermarkets, Mechanical workshops, Office Land- } \\
\text { scapes }\end{array}$ & 750 \\
\hline $\begin{array}{l}\text { Normal drawing work, Detailed mechanical workshops, } \\
\text { Operation theaters }\end{array}$ & 1000 \\
\hline Detailed drawing work, Very detailed mechanical works & $1500-2000$ \\
\hline $\begin{array}{l}\text { Performance of visual tasks of low contrast and very } \\
\text { small size for prolonged periods of time }\end{array}$ & $2000-5000$ \\
\hline Performance of very prolonged and exacting visual tasks & $5000-10000$ \\
\hline $\begin{array}{l}\text { Performance of very special visual tasks of extremely } \\
\text { low contrast and small size }\end{array}$ & $10000-20000$ \\
\hline
\end{tabular}


luminary. Thus, the required illuminance outlined in DIN 5035 indirectly places a mean equality constraint on the optical power (flux) of the luminary.

\section{Peak Intensity}

Up until 2008, LEDs were held to the same eye safety standards, IEC 68025, as coherent light sources such as lasers. While some countries still hold IEC 68025 as the dominant standard, IEC 62471-2006 is now the leading LED luminary eye safety standard in Europe and North America [35]. IEC 62471-2006 specifies four lighting classes, referred to as risk groups (RG) in Table 2.2, for luminaries, such as LEDs. In the case of luminaries intended for general lighting, the distance at which an illuminance of $500 \mathrm{~lx}$ is reached determines the risk group. The further the distance, the higher the risk group. For low intensity sources not intended for illumination, luminaries are classified by their intensity from $200 \mathrm{~mm}$ away.

In practice, due to the large apex angle of LED luminaries, most fall in the exempt or RG-1 category, with very few falling in the RG-2 and RG-3 categories [35]. For example, CREE's LMH2 luminary is capable of producing $3000 \mathrm{~lm}$, the same as two 100 watt incandescent light bulbs, and falls under the exempt risk group [35]. This implies that no peak intensity constraint exists on the majority of luminaries as a result of eye safety standards. Therefore, in most practical cases, the only meaningful peak constraint corresponds to the output intensity generated by the maximum drive current of a luminary. 
Table 2.2: IEC 62471-2006 luminary risk groups [35]

\begin{tabular}{|l|l|l|}
\hline \hline $\begin{array}{l}\text { Risk } \\
\text { Group }\end{array}$ & Risk & Definition \\
\hline Exempt & None & No photobiological hazard \\
\hline RG-1 & Low Risk & $\begin{array}{l}\text { No photobiological hazard under normal behav- } \\
\text { ioral illumination }\end{array}$ \\
\hline RG-2 & $\begin{array}{l}\text { Moderate } \\
\text { Risk }\end{array}$ & $\begin{array}{l}\text { Does not pose a hazard due to human aversion } \\
\text { response to bright light and/or thermal discomfort }\end{array}$ \\
\hline RG-3 & High Risk & Hazardous even for momentary exposure \\
\hline
\end{tabular}

\subsection{The Visible Light Communications Channel}

Figure 2.7 depicts a simplified VLC channel, referred to as an IM/DD channel. In the case of VLC, the transmitters (LEDs) are incoherent optical light sources that are compatible with Intensity Modulation (IM) formats [13, 21, 71, 72]. In IM channels, the instantaneous optical power, flux, of the source, $\Phi_{R}(t)$, is modulated proportional to the driving electrical current, $i_{t}(t)$, which represents the data to be transmitted. The source may be a laser, laser diode, or in the case of VLC, an LED. The optical power signal passes through the channel, free space, and falls upon the receiver elements surface, typically a photodiode. Photodiodes produce an output current, $i_{r}(t)$, directly proportion to the flux incident on the detector surface; this is known as Direct Detection (DD). The current $i_{r}(t)$ is ideally a scaled version the data carrying signal $i_{t}(t)$, and is corrupted by noise.

\subsubsection{Receiver Element: photodiode}

Photodiodes are solid state photoelectric transducers that convert optical signals into electrical signals via the photoelectric effect [71]. The output current, $i_{r}(t)$, is proportional to the flux incident on the photodiodes surface, with the key parameter of 


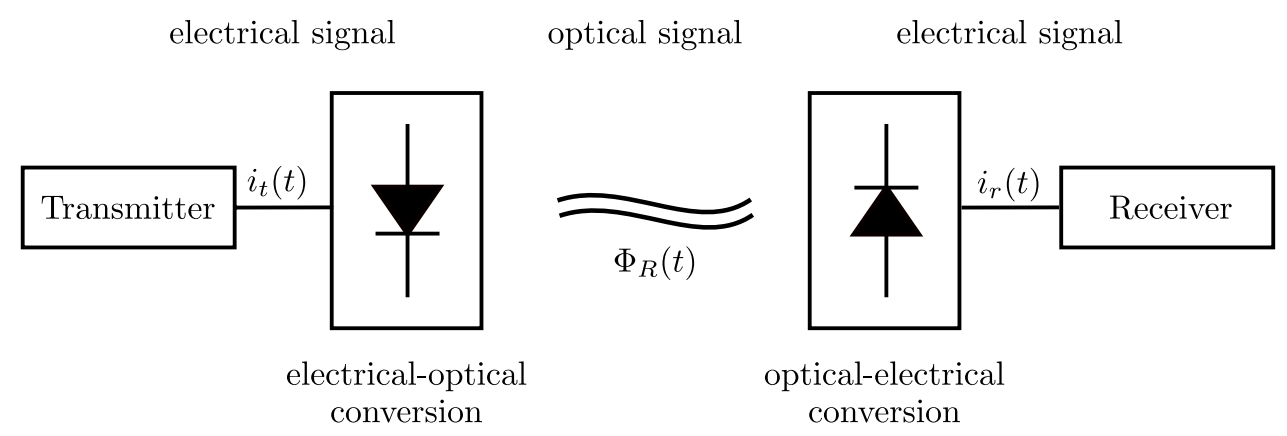

Figure 2.7: IM/DD channel model

a photodiode, responsivity $(R(\lambda))$, given by,

$$
R(\lambda)=\frac{i_{r}}{p(\lambda)}
$$

where $i_{r}$ is the photocurrent generated, and $p(\lambda)$ is the incident optical power at a given wavelength. Responsivity has units (A/W), and depends on both the structure and material of the device. Additionally, $p(\lambda)$, and thus $i_{r}$, are proportional to the effective light collection area of the photodiode. Therefore, to increase the detected power, designers can increase the area of the device. However, this approach is costly and decreases the bandwidth of the device due to larger die capacitance. An alternative method that makes use of an optical concentrator, which focuses light incident on its aperture onto a smaller area, as a means effectively increasing the light gathering area of the photodetector [71].

\subsubsection{Transmitter Element: Light Emitting Diodes}

Light emitting diodes are solid state, current driven, incoherent, semiconductor light sources that emit light through spontaneous emission [71]. When an LED is forward 
biased, recombination of electron-hole pairs in the depletion region releases energy in the form of photons over a range of wavelengths. This effect is known as electroluminescence, where the center wavelength of emitted light is determined by the energy band gap of the semiconductor. Two types of LEDs are commonly used to produce white light $[36,53,54]$. The first type, phosphor LEDs, take an efficient blue LED and coat it with a yellow phosphor to produce white light. The second type, RGB LEDs, produce white light through the additive mixture of red, green, and blue light sources.

\section{Phosphor White Light Emitting Diode}

Figure 2.8 depicts the cross section of a white light phosphor LED. The LED die consists of a blue LED coated with a yellow phosphor, typically cerium doped yttrium aluminum garnet (YAG:Ce) crystals, which produce yellow light when excited [35]. The human eye perceives the combination of blue and yellow light as white light.

A significant challenge in VLC is the efficient utilization of the limited bandwidth of LEDs. When phosphor absorbs energy it takes at set time to release all the energy it stored in the form of yellow light; this effect is known as scintillation. The time for the phosphor to absorb and emit energy is approximately $110 \mathrm{~ns}$ for YAG:Ce, which limits the optical modulation bandwidth of the yellow light emission to about $2 \mathrm{MHz}$ $[73,74]$. However, the blue component of a phosphor LED has an optical modulation bandwidth of approximately $12 \mathrm{MHz}$ [12]. Therefore, by applying a blue filter at the receiver, the bandwidth of the system can be increased to match that of the blue LED at the expense of signal power incident on the receiver.

Another challenge is that the output intensity of an LED has a non-linear relation 


\section{White Light}

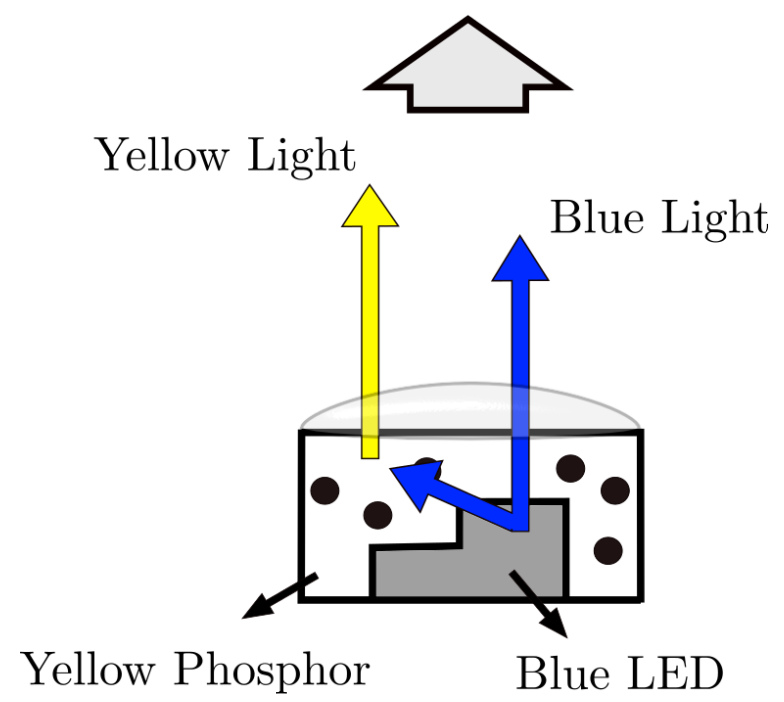

Figure 2.8: Blue LED, yellow phosphor based white LED cross section

to drive current due to a phenomena termed electron leakage [75]. Operating at high currents causes an electric field to develop within the anode region of the diode that allows electrons to escape the active region of the LED. With fewer electrons available for recombination with holes, the number of emitted photons decreases. As non linearities introduce undesirable harmonics into the transmitted signal, designers must create LED drivers capable linearizing the output intensity. Phosphor white LEDs are the most common LED luminaries on the market, with manufactures including OSRAM[54], CREE [35], Philips [36], VISHAY [53], and many more.

\section{Red-Green-Blue Light Emitting Diode}

Another means of producing white light is through the mixing of at least three different narrow-band (colored) light sources [36, 52]. This may be achieved using an RGB LED as seen in Figure 2.9, or by using separate red, green and blue LEDs. Colored 
White Light

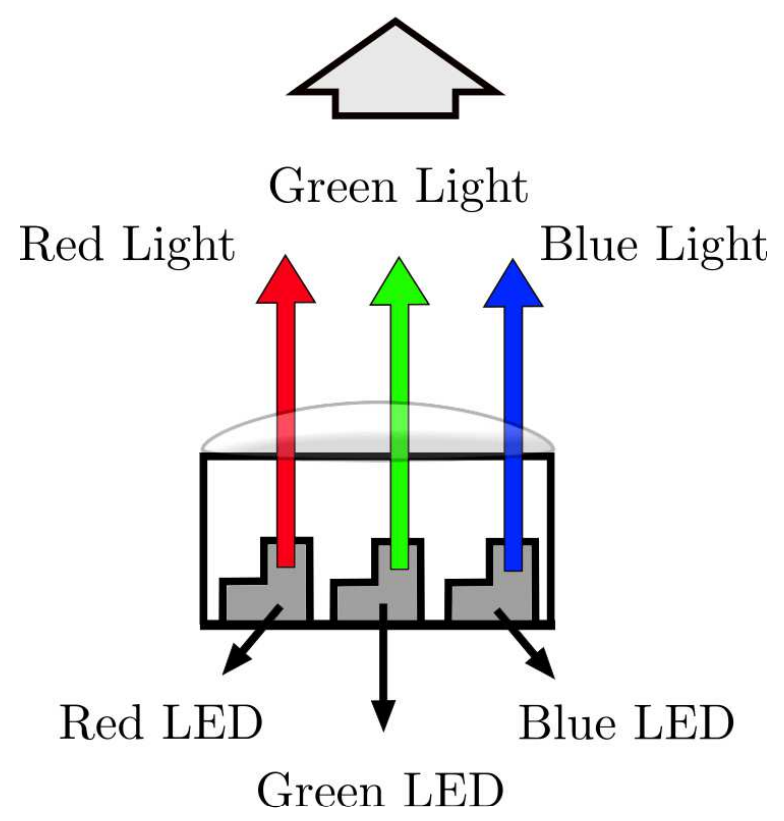

Figure 2.9: RGB LED cross section

LEDs are produced using semiconductors with different band gaps, and may or may not use a phosphor coating to correct their color. Colored LEDs generally have modulation bandwidths greater than that of yellow phosphor LEDs, as they either have no phosphor coating, or their phosphor coating has a shorter decay time.

Unlike phosphor LEDs, the color of the light source can be controlled electronically by individually modulating each color. However, as the emitted intensity of an LED decays with rising temperature, and each LED may not decay at the same rate, the color of the light source will drift. If the drift is significant, additional circuitry is needed to correct the offset [24]. Furthermore, current green LEDs do not convert electrical power into intensity as efficiently as blue and red LEDs. As a result, the lumens per watt of an RGB LED is typically $30 \%$ less than that of a phosphor based LED when producing white light [36]. Thus, RGB LEDs are less favorable in general 
lighting applications. However, development of efficient green LEDs, with and without the use of colored phosphors, are under investigation and show a promising future for RGB LEDs efficiency approaching, and potentially surpassing, that of phosphor coated white light LEDs[33, 34].

\subsubsection{Channel Model}

The VLC channel can be represented by a dispersive additive white Gaussian noise model,

$$
\begin{aligned}
i_{r}(t) & =\bar{R} i_{t}(t) * h_{e o}(t) * h_{c h}(t)+w(t) \\
\Phi_{R}(t) & =i_{t}(t) * h_{e o}(t) \\
\bar{R} & =\int R(\lambda) P(\lambda) \mathrm{d} \lambda
\end{aligned}
$$

where $i_{r}(t)$ is the received signal current, $i_{t}(t)$ is the LED driving current, $h_{e o}(t)$ is the current to flux impulse response of the LED, $h_{c h}(t)$ is the channel impulse response, $w(t)$ is the channel noise, $R(\lambda)$ is the detector responsivity, $\bar{R}$ is the flux to current ratio for a specific $\mathrm{SPD}$, and $*$ represents convolution [76].

Since the system is intensity modulated, $\Phi_{R}(t)$ must be non-negative,

$$
\Phi_{R}(t) \geq 0
$$

Furthermore, as discussed in Section 2.2, the illumination requirement indirectly places an equality constraint, $P_{a v g}$, on the average optical power of the source,

$$
\lim _{T \rightarrow \infty} \frac{1}{2 T} \int_{-T}^{T} \Phi_{R}(t) \mathrm{d} t=P_{a v g}
$$

Lastly, as discussed in Section 2.2, the peak optical power, $P_{\text {peak }}$, of all emissions is 
also bounded by the maximum drive current of the luminary,

$$
\Phi_{R}(t) \leq P_{\text {peak }}
$$

where $P_{\text {peak }}$ represents the LED flux corresponding peak drive current of the device.

\section{Channel Response}

In VLC, the channel, $h_{c h}(t)$, is comprised of a line-of-sight (LOS) component and a diffuse or non-line-of-sight component (NLOS) [28, 76],

$$
h_{c h}(t)=h_{L O S}(t)+h_{D I F F}(t)
$$

The LOS portion, of the channel response, is the signal power received directly from the transmitter, and is modeled by delayed Dirac pulses,

$$
h_{L O S}(t)=\xi_{L O S} \delta\left(t-\Delta t_{L O S}\right)
$$

where $\xi_{L O S}$ and $\Delta t_{L O S}$ are the gain and delay of the LOS signal respectively.

The NLOS portion, of the channel response, comes from the reflections of light from objects present in the environment. As each reflection may arrive at different times with different attenuations, the NLOS component can be modeled by a decaying exponential function in the time domain, and by the following exponential in the frequency domain $[28,77]$,

$$
H_{D I F F}(f)=\xi_{D I F F} \frac{\exp \left(-j 2 \pi f \Delta t_{D I F F}\right)}{1+j f / f_{o}}
$$


where $\xi_{D I F F}$ and $\Delta t_{D I F F}$ are the gain and delay of the diffuse response, and $f_{o}$ is the 3 -dB cut off frequency of the diffuse channel only.

The complete frequency response is given by,

$$
H_{c h}(f)=\xi_{L O S} \exp \left(-j 2 \pi f \Delta t_{L O S}\right)+\xi_{D I F F} \frac{\exp \left(-j 2 \pi f \Delta t_{D I F F}\right)}{1+j f / f_{o}}
$$

where the DC gain is given by,

$$
H_{c h}(0)=\xi_{L O S}+\xi_{D I F F}
$$

When both the LOS and NLOS components are incident on a receiver, the LOS component typically has significantly higher intensity than the NLOS component allowing the NLOS component to be ignored [28]. The LOS gain can be obtained from $[28]$ as,

$$
\xi_{L O S}=A_{r}(m+1) \frac{\cos ^{m}(\theta) \cos (\psi)}{2 \pi r^{2}}
$$

where $A_{r}$ is the effective receiver area, with $r, \Psi$, and $\theta$ are as shown previously in Figure 2.3. An example of an indoor VLC system, with only the LOS component considered, is discussed in Section 2.4.

\section{Channel Noise}

There are two dominant noise sources that need to be considered in direct detection systems, shot noise and thermal noise. Shot noise refers to the random nature of photon absorption and electron hole recombination, modeled by a Poisson distribution, in semiconductor materials. Shot noise in VLC is a result ambient light originating from sources such as sunlight, non-data carrying luminaries, and the mean intensity 
of the data carrying LED luminary itself [76]. While shot noise depends on the received number of photons, and consequently the received intensity of the signal, the average intensity of VLC systems is typically large enough that the shot noise can be considered signal independent. Furthermore, due to high ambient light intensity and the law of large numbers, shot noise can be modeled as an additive white Gaussian noise (AWGN) process as it is result of the summation of many independent Poisson distributed random variables [76].

Thermal noise refers to noise introduced by random, temperature dependent, agitation of charge carries in the receiver electronics. Thermal noise is independent of received intensity signal and is modeled as an additive white Gaussian process [76]. Therefore, the total noise at the receiver can be modeled as an AWGN process, with total variance, $\sigma^{2}$, attributed to both shot and thermal noise [76, 78]. Since the shot and thermal noise are independent, the variance is,

$$
\sigma^{2}=\sigma_{\text {shot }}^{2}+\sigma_{\text {thermal }}^{2}
$$

\section{Signal to Noise Ratio (SNR)}

When considering VLC channels, shot noise tends to dominate VLC channels due the high illuminance requirements outlined in standards like DIN 5035 [13]. This leads to the noise power to be expressed by,

$$
\sigma_{\text {shot }}^{2} \cong N_{\text {shot }} B=q i_{r} B
$$

where $N_{\text {shot }}=q i_{r}$ is the power spectral density of the noise, $i_{r}$ is the induced current in the photodiode caused by ambient light, $q$ is the electron charge and $B$ is the 
effective noise bandwidth [13]. The optical and electrical SNR can then be defined as,

$$
\begin{gathered}
S N R_{o}=\frac{P_{r}}{\sigma} \\
S N R_{e}=\frac{\bar{R}^{2} P_{r}^{2}}{\sigma^{2}}
\end{gathered}
$$

where $P_{r}$ represents average received power, in watts, and is calculated by multiplying the average transmit power, $P_{\text {avg }}$, by $(2.21)$,

$$
P_{r}=H_{c h}(0) P_{a v g}
$$

In VLC channels the $S N R_{e}$ can be in excess of $60 \mathrm{~dB}[12,28]$.

\subsection{Example of an indoor VLC channel}

An example of an indoor VLC channel is provided in order to relate illuminance, a lighting industry requirement, to electrical SNR, an important communications figure of merit. This example is adapted from the work

\footnotetext{
"Broadband Information Broadcasting Using LED-Based Interior Lighting"
}

by Jelena Grubor in [28]. The goal of the study was to determine the available SNR under adherence to the EN 12464-1 illumination standard for work place lighting. Office work, according to EN 12464-1, requires an illuminance of $400 \mathrm{~lx}$ at the work surface. Additionally, the example ensures all other surfaces in the room have an illuminance of at least $200 \mathrm{~lx}$. 
Table 2.3: Philips Luxeon Rebel Specifications of blue emission only [28]

\begin{tabular}{|l|l|}
\hline \hline Characteristic & Value \\
\hline$\theta_{\frac{1}{2}}$ & 60 \\
\hline$P_{\text {avg }}$ & $63 \mathrm{~mW}$ \\
\hline$I_{0}$ & $9.5 \mathrm{~cd}$ \\
\hline
\end{tabular}

The specific LEDs used in this study were not provided, however, the apex angle, radiant flux, and peak luminous intensity are listed in Table 5.1. The LEDs were used to illuminate a room identical to the room shown previously in Figure 2.3. Recall from Section 2.3.3 that noise power is dominated by the shot noise induced by ambient light. In this experiment, after applying a blue filter at the receiver, the ambient light in the room produced a DC photocurrent of $0.62 \mathrm{~mA}$. Therefore, the noise power spectral density, with units $\mathrm{A}^{2} / \mathrm{Hz}$ is given as,

$$
N_{o} \approx N_{\text {shot }}=q I=10^{-22}
$$

and the total noise power, with units $\mathrm{A}^{2}$ is approximately,

$$
\sigma^{2} \approx \sigma_{\text {shot }}^{2}=N_{o} B=2 \times 10^{-15}
$$

where the receive bandwidth, $B$, is assumed to be equal to the blue filtered LED modulation bandwidth of $12 \mathrm{MHz}$.

The total illuminance at the work surface, and thus the total received power $P_{r}$, is equal to the sum of the illuminance and received power of each LED in the lighting fixture. As discussed in Section 2.3.3, the receive power, $P_{r}$, is dominated by the LOS 
signal component of the channel response,

$$
\begin{aligned}
& P_{r}=\sum_{i=1}^{N_{L E D}} H_{i}(0) P_{a v g} \\
& H_{i}(0)=\eta_{L O S, i}
\end{aligned}
$$

where $N_{L E D}$ is the number of LEDs for a given scenario. In order to relate receive power to illuminance, combine (2.22) and (2.30),

$$
P_{r}=P_{\text {avg }} \sum_{i=1}^{N_{L E D}} \eta_{L O S_{i}}=P_{\text {avg }} A_{r}(m+1) \sum_{i=1}^{N_{L E D}} \frac{\cos ^{m}\left(\theta_{i}\right) \cos \left(\Psi_{i}\right)}{2 \pi r_{i}^{2}}
$$

Recall from (2.32), that horizontal illuminance of a single LED is expressed by,

$$
E_{h}=I_{0} \frac{\cos ^{m}(\theta) \cos (\Psi)}{r^{2}}
$$

Therefore,

$$
\begin{aligned}
P_{r} & =\alpha A_{R} E_{h} \\
\alpha & =\frac{P_{\text {avg }}(m+1)}{2 \pi I_{0}}
\end{aligned}
$$

This leads to the electrical $S N R, S N R_{e}$, to be expressed as,

$$
S N R_{e}=\frac{\left(R E_{h} A_{R} \alpha\right)^{2}}{N_{o} B}
$$

In this experiment, the photodetector has a responsivity of $R=0.28 \mathrm{~A} / \mathrm{W}$ for the received spectrum, and an effective receive area of $A_{R}=3 \mathrm{~cm}^{2}$. Additionally, $m=1$, $I_{0}=9.5 \mathrm{~cd}, P_{\text {avg }}=0.63 \mathrm{~mW}, N_{o} B=2 \times 10^{-15}$ and $E_{h}=400 \mathrm{~lx}$. Substituting the aforementioned values into (2.34) leads to an electrical SNR of $59.5 \mathrm{~dB}$ on a surface with an illuminance of $400 \mathrm{~lx}$. 


\subsection{Compatible VLC Modulation Techniques}

As previously discussed, IM/DD is the only transmission scheme compatible with incoherent light sources, such as LEDs. As a result, the phase of the optical carrier cannot be modulated and the modulating electrical signal must be non-negative. Therefore, the direct use of traditional radio modulation schemes is not possible. There exist several IM/DD compatible modulation schemes that are under study for use in VLC, three of which, have been standardized by the IEEE $[12,24,79,80]$. Onoff keying (OOK), variable pulse position modulation (VPPM), and color-shift keying (CSK) are the standardized VLC modulation formats. Additionally, metameric modulation (MCM) and multiple sub-carrier modulation (MSM) are under investigation as potential modulation formats for VLC. Brief overviews of OOK, VPPM, MCM, and MSM are provided and are followed by a detailed treatment of CSK.

\subsubsection{On-Off Keying}

On-Off Keying is the most commonly used IM/DD modulation scheme due to its simple implementation. Figure 2.10 depicts an example of two different OOK pulses. In OOK, data are sent via the presence or absence of light. The simplest form of OOK is referred to non-return-to-zero (NRZ) OOK, where a binary "one" is represented by a light pulse, and binary "zero" with no light. In IEEE 802.15.7 section 8.2, OOK is proposed using Manchester symbols instead of NRZ. In Manchester code, a binary "one" is represented by no light followed by a light pulse, and a binary "zero" is represented by a light pulse followed by no light. Manchester coded symbols use twice the bandwidth of NRZ pulses, and therefore are less attractive for high speed communication in limited bandwidth channels, such as VLC channels. However, 


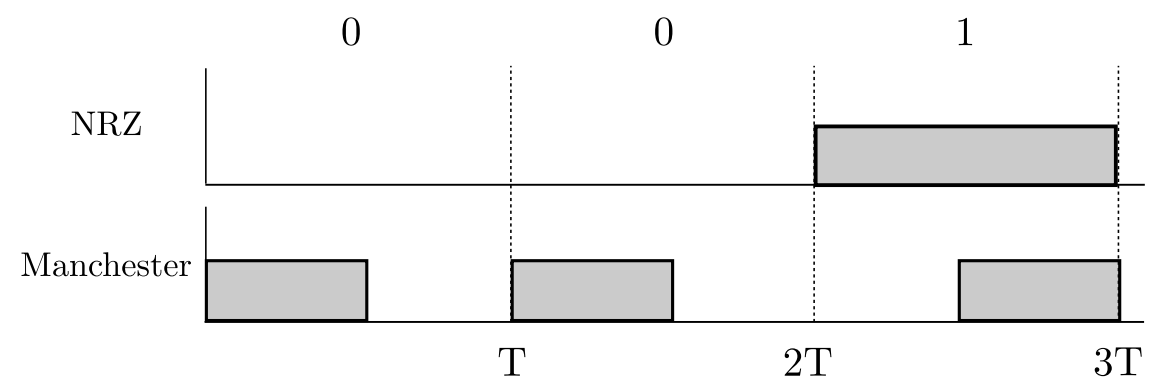

Figure 2.10: Example of NRZ OOK (top) and Manchester OOK(bottom)

Manchester symbols have equal mean, therefore, unlike NRZ, Manchester symbols are less likely inject data dependent flicker into the system.

\subsubsection{Variable Pulse Position Modulation}

Variable pulse position modulation is a VLC modulation scheme outlined in IEEE 802.15.7 section 8.2. Figure 2.11 depicts examples of VPPM symbols. VPPM is a combination of binary pulse position modulation, to prevent data dependent flicker, and pulse width modulation to control the intensity of a luminary. Bits in VPPM are distinguished by the pulse position during the symbol period. Binary "zero" occurs when the pulse is aligned to the left of the symbol period and binary "one" occurs when the pulse is aligned to the right of the symbol period. The width of the pulse can be adjusted to reduce the average intensity of the source, while maintaining that the mean of binary "one" and binary "zero" are equal. Since each VPPM symbol, for a given dimming level, has equal mean, the data is unlikely to produce flicker. In essence, VPPM is Manchester OOK with a variable duty cycle, and has equally poor spectral efficiency. 


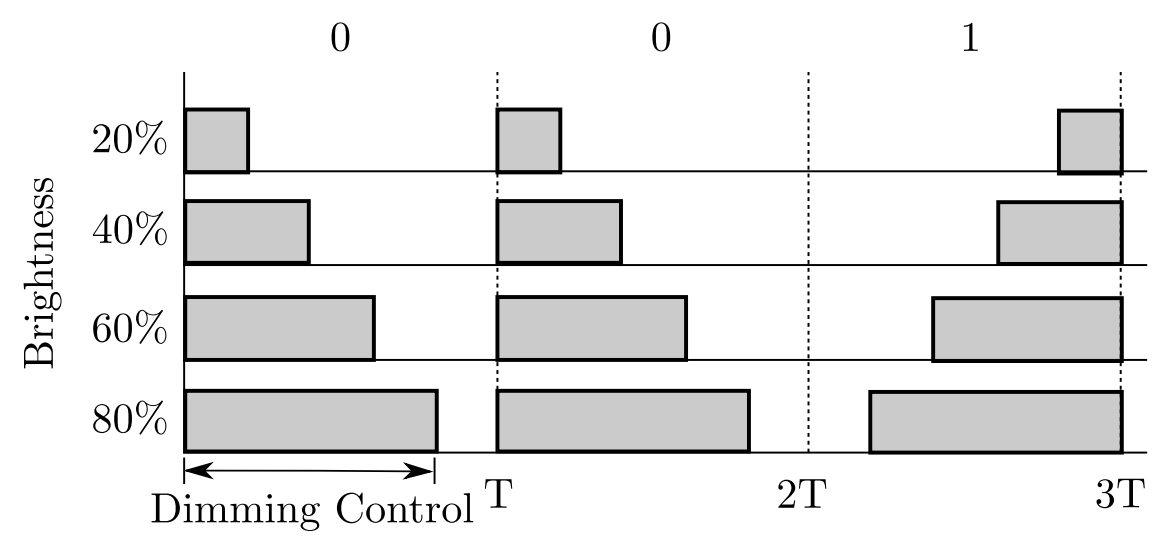

Figure 2.11: Example of VPPM data pulses

\subsubsection{Multiple Sub-Carrier Modulation}

In addition to single carrier modulation methods, such as VPPM and OOK, VLC is also compatible with multiple sub-carrier modulation (MSM) techniques. MSM techniques are modulation schemes where information is modulated onto orthogonal sub-carriers. The sum of the modulated sub-carriers is then modulated onto the instantaneous power of the transmitter. Due to the orthogonality of the sub-carriers, MSM is well-suited for the simultaneous transmission of data from a single transmitter hub to several receivers. Furthermore, by simultaneous transmission of several narrow-band sub-carriers, MSM can achieve the same data rates as single carrier modulation with simple frequency domain equalizers compared to the complex time domain equalizers needed in single carrier modulation formats. However, MCM has the problem of high peak to average power ratios that require inefficient, highly linear, driving circuitry. Currently, there are no multi-carrier schemes proposed in VLC standards, however a topic of interest in optical wireless research is that of orthogonal frequency division multiplexing (OFDM) [79, 80, 81]. 


\subsubsection{Metameric Modulation}

Metameric modulation is VLC specific modulation scheme that exploits a human vision phenomena known as metamerism. Metamerism refers to the ability to produce a specific perceived color with more than one SPD. Metameric modulation requires the use of multiple colored LEDs with at least four different chromaticities. Symbols are selected such that the output color of the light source remains fixed, regardless of data symbol sent, while the luminous flux of each symbol varies.

In the case of four distinct chromaticities, two groups of three chromaticities are selected such that the area each group covers in the $x y$ chromaticity space overlaps. Figure 2.12 demonstrates the use of two chromaticity groups with the veriticies of each group labeled as 1 and 2; the dot near the center of the diagram represents the fixed operating chromaticity. Each group can be independently modulated to produce an output chromaticity such that the sum of the chromaticities of the two groups always produces the same desired operating chromaticity. Even though only four unique chromaticities exist, this process requires requires the use of six independently controllable LEDs, where two of the colored LEDs are used twice. The primary strength of metameric modulation is that it supports higher order constellations allowing it to exploit the high SNR of VLC channels to gain spectral efficiency. Another strength of metameric modulation is that, theoretically, the color of the light source will not drift in the event of long strings of a single repeated data symbol.

\subsubsection{Color-Shift Keying}

Color-shift keying is a VLC specific modulation scheme, specified in section 8.2 of IEEE 802.15.7, designed to operate with RGB LEDs in order to provide higher order, 


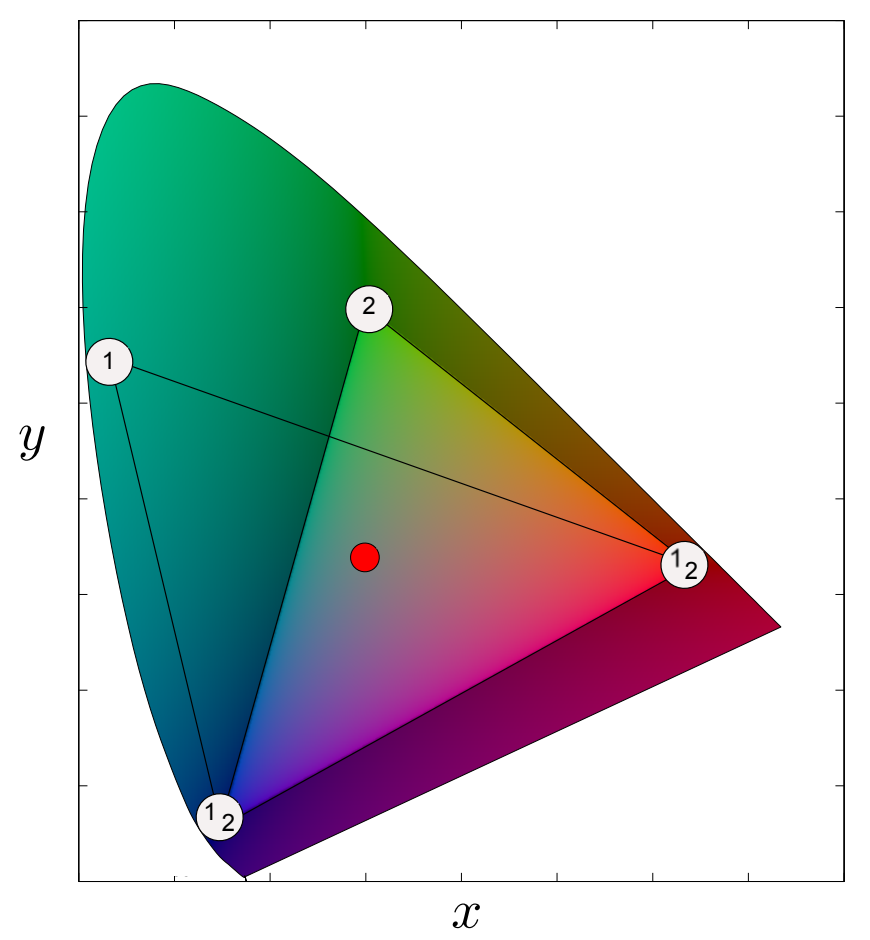

Figure 2.12: Example of metameric modulation in the $x y$ chromaticity space with two LED sets 


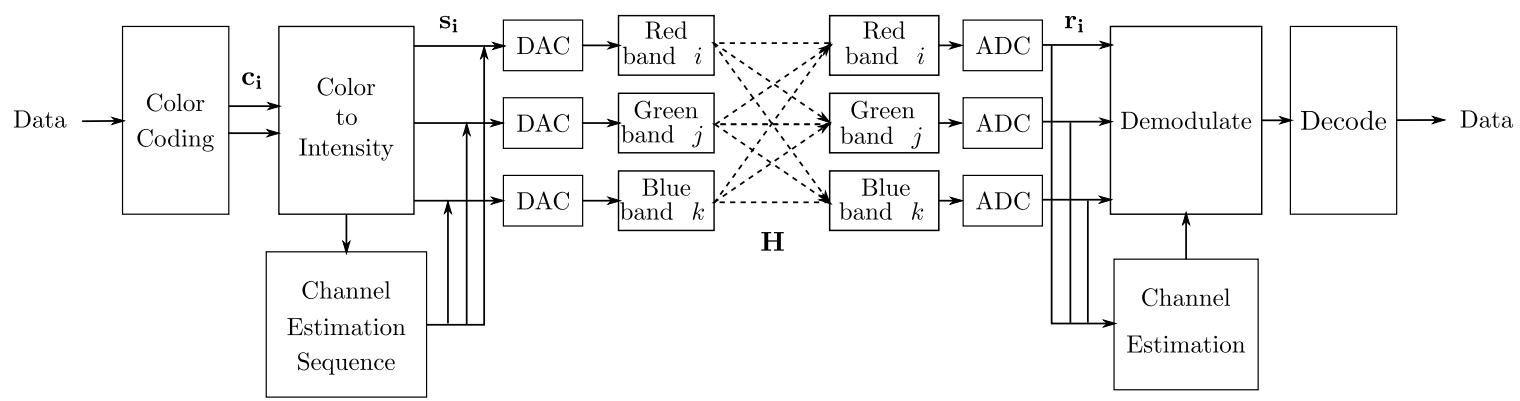

Figure 2.13: Functional block diagram of VLC channel that supports the CSK modulation format

spectrally efficient modulation. Unlike metameric modulation, symbols are selected such that the source luminous flux remains constant while its instantaneous chromaticity varies [24]. Thus, data are sent on the instantaneous color of the RGB triplet, while maintaining an average perceived chromaticity. The color based modulation of CSK has two advantages over intensity modulated schemes. The first advantage is the constant luminous flux guarantees an absence of flicker at all frequencies. The second advantage is that the constant luminous flux of the source leads to near constant current drive, which in turn implies a reduced inrush current when modulating data, strong signal isolation from the power line and a reduction in inductance caused by large switching currents.

Figure 2.13 depicts a functional block diagram of a typical CSK compatible VLC link. Data are first mapped onto the constellation,

$$
\mathcal{A}=\left\{\mathbf{c}_{1}, \mathbf{c}_{2}, \mathbf{c}_{3}, \ldots, \mathbf{c}_{\mathrm{N}}\right\}
$$

where $N$ is the constellation size and each symbol, $\mathbf{c}_{\mathbf{i}}$, is a member of the CIE 1931 $x y$ chromaticity gamut,

$$
\mathbf{c}_{\mathbf{i}}=\left[x_{c_{i}}, y_{c_{i}}\right]
$$


with $x_{c_{i}}$ and $y_{c_{i}}$ representing the $x y$ chromaticity of the symbol. The set of symbols, $\mathbf{c}_{\mathbf{i}}$, are then converted into the absolute luminous flux symbol set, $\mathbf{s}_{\mathbf{i}}$,

$$
\mathbf{s}_{\mathbf{i}}=\left[l_{r}, l_{g}, l_{b}\right]
$$

with $l_{r}, l_{g}$ and $l_{b}$ representing the absolute luminous flux of the red, green and blue color bands respectively. Applying (2.10) to the notion of color mixing described in (2.11), the relationship between $\mathbf{c}_{\mathbf{i}}$ and $\mathbf{s}_{\mathbf{i}}$ as,

$$
\left[\begin{array}{ccc}
\frac{X_{r}}{Y_{r}} & \frac{X_{g}}{Y_{g}} & \frac{X_{b}}{Y_{b}} \\
\frac{Y_{r}}{Y_{r}} & \frac{Y_{g}}{Y_{g}} & \frac{Y_{b}}{Y_{b}} \\
\frac{Z_{r}}{Y_{r}} & \frac{Z_{g}}{Y_{g}} & \frac{Z_{b}}{Y_{b}}
\end{array}\right] \mathbf{S}_{\mathbf{i}}=L\left[\begin{array}{c}
\frac{x_{c_{i}}}{y_{c_{i}}} \\
1 \\
\frac{1-x_{c_{i}}-y_{c_{i}}}{y_{c_{i}}}
\end{array}\right]
$$

where $L$ is the desired average luminous flux of the luminary. The use of digital to analog converters (DAC) and analog current drivers then produce the appropriate current levels required to generate the appropriate luminous flux of each symbol. In order to determine the required drive current from $l_{r}, l_{g}$ and $l_{b}$, the lumens per ampere of each LED must be known.

Following the electrical to optical conversion, the intensity signal passes through free space and is detected by a set of three filtered photodetectors, where it is corrupted by AWGN. Thus, a VLC channel that supports CSK is simply three IM/DD channels, as discussed in Section 2.3.3, related by the channel gain matrix,

$$
\mathbf{H}=\left[\begin{array}{lll}
h_{1,1} & h_{1,2} & h_{1,3} \\
h_{2,1} & h_{2,2} & h_{2,3} \\
h_{3,1} & h_{3,2} & h_{3,3}
\end{array}\right]
$$


where $h_{i, j}$ represents the channel gain, including the transmitter and receiver conversions between optical and electrical domains, between transmit band, $j$, and receive band, $i$. The elements of $\mathbf{H}$ depend on both the, potentially overlapping, SPDs of each LED and the responsivities of the filtered photodetectors. As in Section 2.3.3, only the LOS component of the free space channel is considered; therefore, the equivalent discrete time baseband channel model is,

$$
\mathbf{r}_{\mathbf{i}}=\left[p_{r}, p_{g}, p_{b}\right]=\mathbf{H s}_{\mathbf{i}}+\mathbf{n}_{\mathbf{i}}
$$

where $\mathbf{r}_{\mathbf{i}}$ is the received symbol, $p_{r}, p_{g}$ and $p_{b}$ are the outputs of the photodetectors following analog to digital conversion, and $\mathbf{n}_{\mathbf{i}}$ is the AWGN vector. The channel matrix is assumed to be deterministic and invertible, and is either provided as part of the specification, or estimated by a channel estimation sequence. Using the estimated channel matrix, the receiver performs maximum likelihood detection on the received signal, and the final block converts the decoded symbol back into its $x y$ chromaticity coordinates.

\subsection{Standardized CSK Constellation Design Rules}

Section 12.8 of IEEE 802.15.7 specifies design rules for constellations of 4,8 and 16 symbols[24]. The design rules map constellation points over the entire gamut of an RGB LED, and do not consider the resulting chromaticity of the luminary. Furthermore, the design rules are independent of the channel gain matrix. These design rules will act as the bench mark for the constellation design method introduced in Chapter 3 when comparing symbol error performance in Chapter 5. 


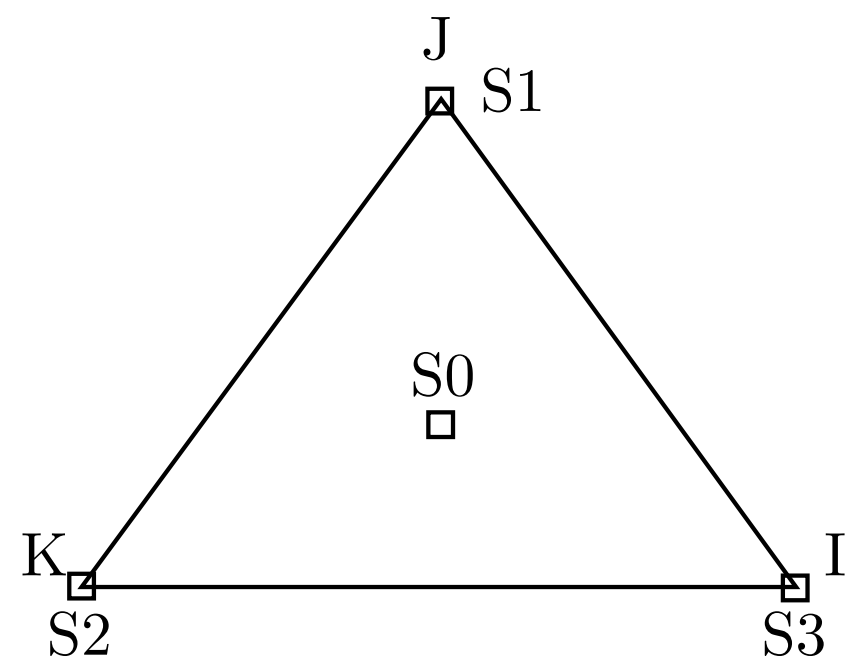

Figure 2.14: IEEE Standard 802.15.7 4-CSK design rule [24]

\subsubsection{4-CSK}

The design rule for 4-CSK, as outlined in IEEE 802.15.7, is shown in Figure 2.14. Elements S0 through S3 are the symbols, $\mathbf{c}_{\mathbf{i}}$, of 4-CSK. Points I, J, and K are the $x y$ chromaticity coordinates of the red, green and blue LEDs. Elements S1, S2, and S3 are three vertices's of the triangle IJK. Element S0 is the centroid of the triangle IJK

\subsubsection{8-CSK}

The design rule for 8-CSK, as outlined in IEEE 802.15.7, is shown in Figure 2.15. Elements S0 through S7 are the symbols, $\mathbf{c}_{\mathbf{i}}$, of 8-CSK. Points I, J, and K are the $x y$ chromaticity coordinates of the red, green and blue LEDs. Elements S0, S4, and S7 are three vertices's of the triangle IJK. Elements S1 and S2 are the points that divide side $\mathrm{JK}$ and side $\mathrm{JI}$ in the ratio 1:2. Points $\mathrm{B}$ and $\mathrm{C}$ are midpoints of the line JI and line JK respectively. Element S6 is the midpoint of the line KI. Point A is the centroid of the triangle B-S6-I. Point D is the centroid of the triangle C-K-S6. 


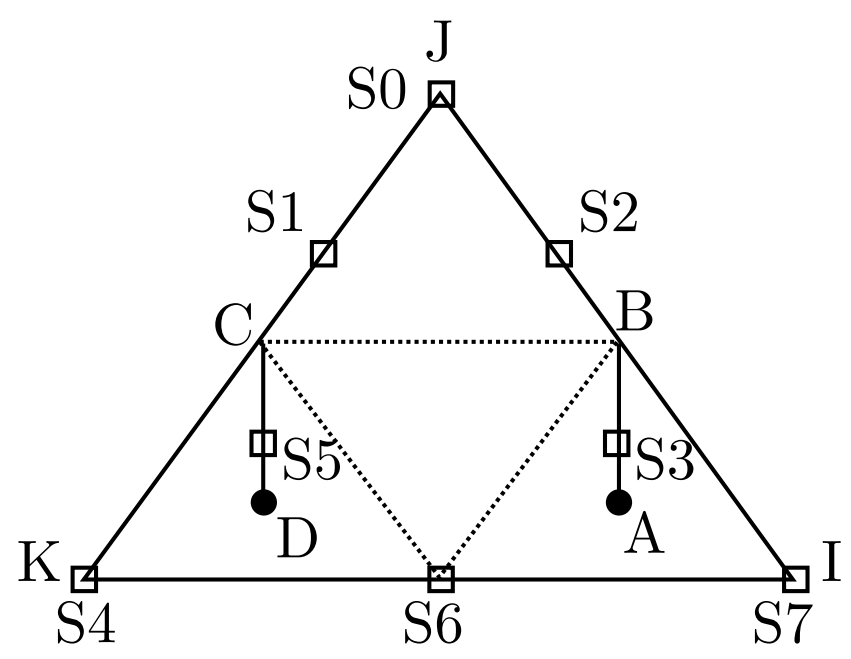

Figure 2.15: IEEE Standard 802.15.7 8-CSK design rule [24]

Element $\mathrm{S} 3$ is the point that divides line $\mathrm{AB}$ in the ratio 1:2. Element S5 is a point that divides line $\mathrm{DC}$ in the ratio 1:2.

\subsubsection{6-CSK}

The design rule for 16-CSK, as outlined in IEEE 802.15.7, is shown in Figure 2.16. Elements S0 through S15 are the symbols, $\mathbf{c}_{\mathbf{i}}$, of 16-CSK. Points I, J, and K are the $x y$ chromaticity coordinates of the red, green and blue LEDs. Elements S2 and S8 are points that divide side JK in one third. Elements S3 and S12 are points that divide side JI in one third. Elements S11 and S14 are points that divide side KI in one third. Element S0 is the centroid of the triangle IJK. Elements S1, S4, S6, S7, S9, and S13 are the centroids of each of the smaller triangles seen in Figure 2.16. 


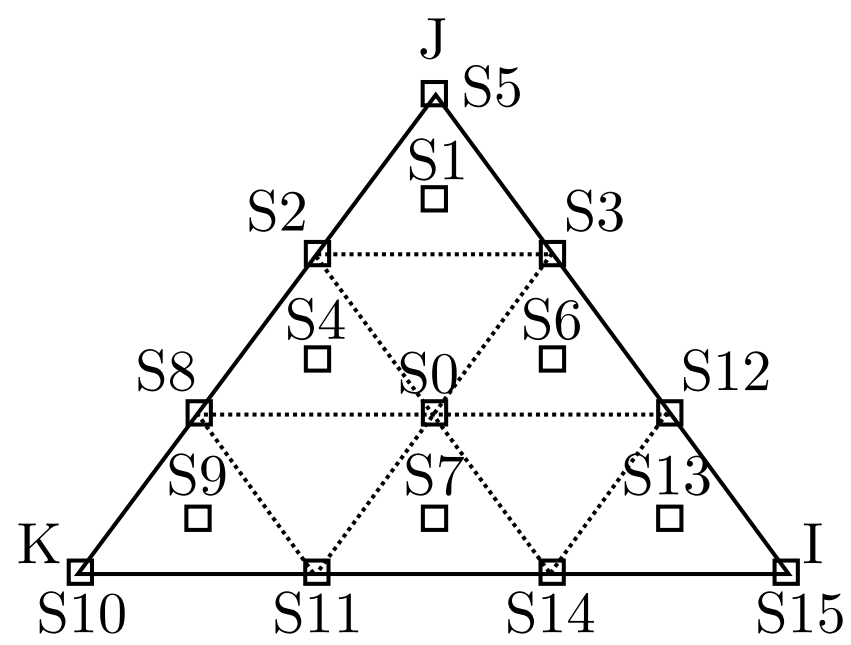

Figure 2.16: IEEE Standard 802.15.7 16-CSK design rule [24]

\subsection{Conclusions}

In this chapter, the VLC channel was shown to have limited bandwidth, high SNR and features little to no multi-path distortion. The discussion suggest the use of bandwidth efficient modulation, such as CSK, is required in order to achieve high data rates. However, the current CSK design rules do not account for the human color perception of the light source, nor do they attempt to compensate for crosstalk between the color channels. In the following chapter, a novel set of design rules for CSK, based on deterministic convex optimization, is introduced and analyzed. The proposed method is capable of designing CSK constellations that produce a specified operating color and intensity, and optimizes the constellation based on the estimated channel matrix. Furthermore, while IEEE 802.15.7 specifies that CSK constellations be designed such that they produce a fixed luminous intensity, it was shown that the average human does not perceive flicker above $60 \mathrm{~Hz}$, and that no negative biological effects occur above $200 \mathrm{~Hz}$. This suggests that with proper data scrambling and high symbol rates, it is not necessary for the source to operate with a fixed luminous 
intensity. In fact, as discussed in Section 2.5.5, there is great benefit to holding the drive current constant. Therefore, Chapter 3 also provides a modification to the optimization problem when constant current is of interest. 


\section{Chapter 3}

\section{Designing CSK Constellations}

In this chapter, a rigorous design framework for CSK constellations, operating either with constant luminous flux or constant electrical current, based on convex optimization is presented. The benefits of the approach are that it can produce constellations of any size while satisfying peak and average intensity constraints, compensating for cross talk between each color channel, and permits the selection of an operating chromaticity in order to meet lighting industry quality standards. Constellation design examples over various constraints are presented, and an efficient heuristic for designing large constant constellations is provided. The chapter concludes with the relaxation of the constant flux/current constraints to allow for a specified ripple in the flux/current. The relaxation allows three dimensional (3D) CSK constellations to be designed that exhibit better communication properties than conventional CSK constellations. 


\subsection{Optimization Constraints}

In Section 2.5.5, it was shown that CSK is intended to operate with a constant luminous flux to prevent flicker. Furthermore, Section 2.2 stated all luminaries sold in North America must pass the Energy Star certification. One requirement of the certification is that the light source produce one of the standard correlated color temperatures. As the current IEEE 802.15.7 design rules do not account for the perceived color of the light source, it is unlikely these rules will provide a standardized color temperature for all possible red, green and blue LEDs available on the market. Therefore, an additional constraint must be placed on the mean of the CSK alphabet to enable control of the light sources chromaticity. Furthermore, as humans cannot perceive the instantaneous color of a symbol, this chapter describes symbols by their three luminous flux components, $l_{r}, l_{g}$ and $l_{b}$, rather than chromaticity. Finally, as the usefulness of the CRI is limited for LEDs, and there exists no consensus on a new color rendering metric for LEDs, subsequent sections do not consider color rendering metrics.

\subsubsection{Luminous Flux Constraints}

Since CSK operates with a fixed luminous flux, symbols are selected such that,

$$
l_{r}+l_{g}+l_{b}=L>0
$$


where $L$ is the desired total operating luminous flux. Furthermore, luminous flux is non-negative and each color band has a peak, possibly different, luminous flux

$$
0 \leq l_{r, g, b} \leq L_{r, g, b}
$$

where $L_{r, g, b}$ are the peak luminous flux of each channel. The constraints on $\mathbf{s}_{\mathbf{i}}$ represent a convex polygon in $\mathbb{R}_{+}^{3}$, where each point on the polygon represents a different chromaticity. At the transmitter the polygon is an equilateral triangle. However each vertex of the triangle can be truncated if any of $L_{r, g, b}<L$, resulting in a three to six sided convex polygon. Due to the channel matrix, $\mathbf{H}$, defined in (2.39), the receiver perceives a scaled version of this polygon. Therefore, the signal set $\mathbf{s}_{\mathbf{i}}$ is determined by designing a new set, $\tilde{\mathbf{s}_{\mathbf{i}}}$, in the distorted receive polygon, with $\mathbf{s}_{\mathbf{i}}$ and $\tilde{\mathbf{s}_{\mathbf{i}}}$ related by,

$$
\tilde{\mathbf{s}_{\mathbf{i}}}=\mathbf{H s}_{\mathbf{i}}
$$

Since the design region of $\tilde{\mathbf{s}_{\mathbf{i}}}$ is a plane, the problem can be simplified to two dimensions by scaling the polygon by $L$, taking the orthographic projection transformation of the plane, viewing along its normal, and truncating the dimension with a constant value for all symbols. The orthographic projection can be thought of as rotating the plane such that its normal aligns with the $\mathrm{Z}$ axis, and then truncating the $\mathrm{Z}$ dimension. This will produce a convex polygon in $\mathbb{R}^{2}$, which is termed the normalized design space. All points of the normalized design space have the same luminous flux. The orthographic projection transform is invertible, provided the truncated dimension is preserved elsewhere. Therefore any point in the normalized design space has a oneto-one mapping with a point in the polygon in $\mathbb{R}_{+}^{3}$, perceived by the receiver. As the 
projection transform does not color AWGN, the problem is equivalent to optimizing for the set of points in this new region, denoted by the set $\phi_{i}$, with,

$$
\phi_{i}=\frac{1}{L} \mathbf{T} \mathbf{P}_{\mathbf{r}} \tilde{\mathbf{s}_{\mathbf{i}}}
$$

where $\mathbf{P}_{\mathbf{r}}$ is the orthographic projection matrix, and $\mathbf{T}$ represents the dimension truncation. The orthographic projection matrix used is this study is defined by the use of two rotation matrices with the form,

$$
\begin{aligned}
& \mathbf{R}_{\mathbf{z}}=\left[\begin{array}{ccc}
\cos (a z) & -\sin (a z) & 0 \\
\sin (a z) & \cos (a z) & 0 \\
0 & 0 & 1
\end{array}\right] \\
& \mathbf{R}_{\mathbf{x}}=\left[\begin{array}{ccc}
1 & 0 & 0 \\
0 & \cos (e l) & -\sin (e l) \\
0 & \sin (e l) & \cos (e l)
\end{array}\right]
\end{aligned}
$$

where $\mathbf{P}_{\mathbf{r}}=\mathbf{R}_{\mathbf{x}} \mathbf{R}_{\mathbf{z}}$ and the angles of rotation, $e l$ and $a z$, are the elevation and azimuth angles of the normal of the plane defined by the points formed by the columns of $\mathbf{H}$.

In order to define the constraint region in a form used by most commercial optimization tools, it must be expressed as a set of linear inequalities. This is equivalent to the intersection of half spaces, defined by the linear inequality,

$$
\mathbf{A} \phi_{\mathbf{i}} \leq \mathbf{b}
$$

where the rows of $\mathbf{A}$ are the outward facing normal vectors of each side of the polygon, 
and the elements of $\mathbf{b}$ are the orthogonal Euclidean distances of each side from the origin. To simplify the notation further, let

$$
\bar{\phi}=\operatorname{vec}\left(\phi_{\mathbf{i}}\right)
$$

where vec represents the column wise vectorization. Furthermore, let

$$
\overline{\mathbf{b}}=\left[\begin{array}{c}
\mathbf{b} \\
\mathbf{b} \\
\vdots \\
\mathbf{b}
\end{array}\right]
$$

and,

$$
\overline{\mathbf{A}}=\left[\begin{array}{cccc}
\mathbf{A} & 0 & 0 & 0 \\
0 & \mathbf{A} & 0 & 0 \\
0 & 0 & \ddots & 0 \\
0 & 0 & 0 & \mathbf{A}
\end{array}\right]
$$

leading the inequality constraints to be expressed as,

$$
\overline{\mathbf{A}} \bar{\phi} \leq \overline{\mathbf{b}}
$$




\subsubsection{Chromaticity Constraint}

In order to fix the perceived chromaticity of the source, an average constraint is required on the symbol alphabet, and is expressed by the affine equality,

$$
\sum_{i} w_{i} \mathbf{s}_{\mathbf{i}}=\mathbf{d}
$$

where $w_{i}$ is the probability of $\mathbf{s}_{\mathbf{i}}$, and $\mathbf{d}$, defined previously in (2.11), is a vector whose elements represent the average luminous flux of each color band needed to generate the desired chromaticity. When $\mathbf{d}$ is not specified, no color constraint exists and the alphabet is said to have no color balance.

In order to design $\phi_{\mathbf{i}}$ around $\mathbf{d}$, the transform in equation (3.4) must be applied to $\mathbf{d}$ to form the point $\mathbf{d}^{*}$. In standard matrix form, assuming equally likely symbols, the average color constraint is given by the affine equality,

$$
\mathbf{C} \bar{\phi}=\mathbf{d}^{*}
$$

where $\bar{\Phi}$ is the vectorization of all symbols $\phi_{i}$, and $\mathbf{C}$ is formed by the $N$ fold horizontal replication of the $2 \times 2$ identity matrix normalized by $N$.

\subsubsection{Modification for constant current}

Opting for constant current instead of constant intensity merely scales the color constraint and the channel matrix, both of which vary based on the LEDs used in the system. Therefore, the use of constant current does not disqualify the validity of the design methods themselves. In order to modify the luminous flux constraints to 
represent constant current, all the luminous flux values in (3.1) and (3.2) are simply relabeled to represent current while the form of the constraints remains unchanged. Additionally, the channel matrix will now represent the gain from the current driven into an LED to the output voltage of an amplified photo detector. The normalized design space for constant current is then calculated exactly as described by (3.4).

While the chromaticity constraint remains unchanged, the elements of $\mathbf{d}$ must be scaled to produce the mean current constraint vector $\mathbf{d}_{\mathbf{c}}$,

$$
\mathbf{d}_{\mathbf{c}}=\left(\left[\begin{array}{lll}
\eta_{r}^{-1} & \eta_{g}^{-1} & \eta_{b}^{-1}
\end{array}\right] \mathbf{d}\right)^{-1}\left[\begin{array}{ccc}
\eta_{r} & 0 & 0 \\
0 & \eta_{g} & 0 \\
0 & 0 & \eta_{b}
\end{array}\right]^{-1} \mathbf{d}
$$

where $\eta_{r}, \eta_{g}$ and $\eta_{b}$ represent the lumen's per ampere for the red, green and blue LEDs respectively. The term in front of the diagonal scaling matrix normalizes the elements of $\mathbf{d}_{\mathbf{c}}$ such that they the sum to unity, thus representing the relative mean current of each LED required to produce the desired chromaticity. The new mean constraint is nearly identical to equation (3.15) and is given by,

$$
\mathbf{C} \bar{\phi}=\mathbf{d}_{\mathbf{c}}^{*}
$$

where $\mathbf{d}_{\mathbf{c}}^{*}$ is the representation of $\mathbf{d}_{\mathbf{c}}$ in the normalized design space.

\section{$3.2 \quad$ Objective}

It is well known when a high SNR signal is corrupted by AWGN, as is the case for VLC as observed in Section 2.4, that the symbol error rate (SER) is directly related 
to the minimum pairwise Euclidean distance between all symbols in the alphabet. In order to minimize the SER for a given SNR and rate, the minimum distance between all CSK symbols, as seen be the receiver, must therefore be maximized,

$$
\arg \max _{\phi_{\mathbf{i}}} \min _{i \neq j}\left\{\left\|\phi_{\mathbf{i}}-\phi_{\mathbf{j}}\right\|_{2}\right\}
$$

The primary challenge with this objective is that it is non-convex; therefore, optimization algorithms are only capable of recognizing local maximizers without knowledge of whether or not the solution achieved is a global maximizer. Furthermore, the local maximizer obtained by any algorithm depends on the initial estimate used to start the optimization process. However, the optimal solution is non unique; for any optimal solution another, equally optimal, solution can be obtained by swapping any two points in the constellation. The aforementioned property of the objective increases the likelihood of discovering a globally optimal solution from optimizing the objective multiple times over a small set of random starting points.

Another challenge with the objective is that it does not lend itself to gradient based optimization techniques as the minimum function is discontinuous. The nondifferentiable nature of the objective can be addressed by considering a continuous approximation to the minimum function. This is achieved using well known "logsum-exponentials" continuous approximation,

$$
\min _{i}\left\{d_{i}\right\}=-\ln \left(\sum_{i} \exp \left(-\beta d_{i}\right)\right) / \beta
$$

where $d_{i}$ is a set of distances and $\beta$ is positive scalar [82]. Provided one of the $d_{i}$ is sufficiently small compared to the others, this function returns an approximation to 


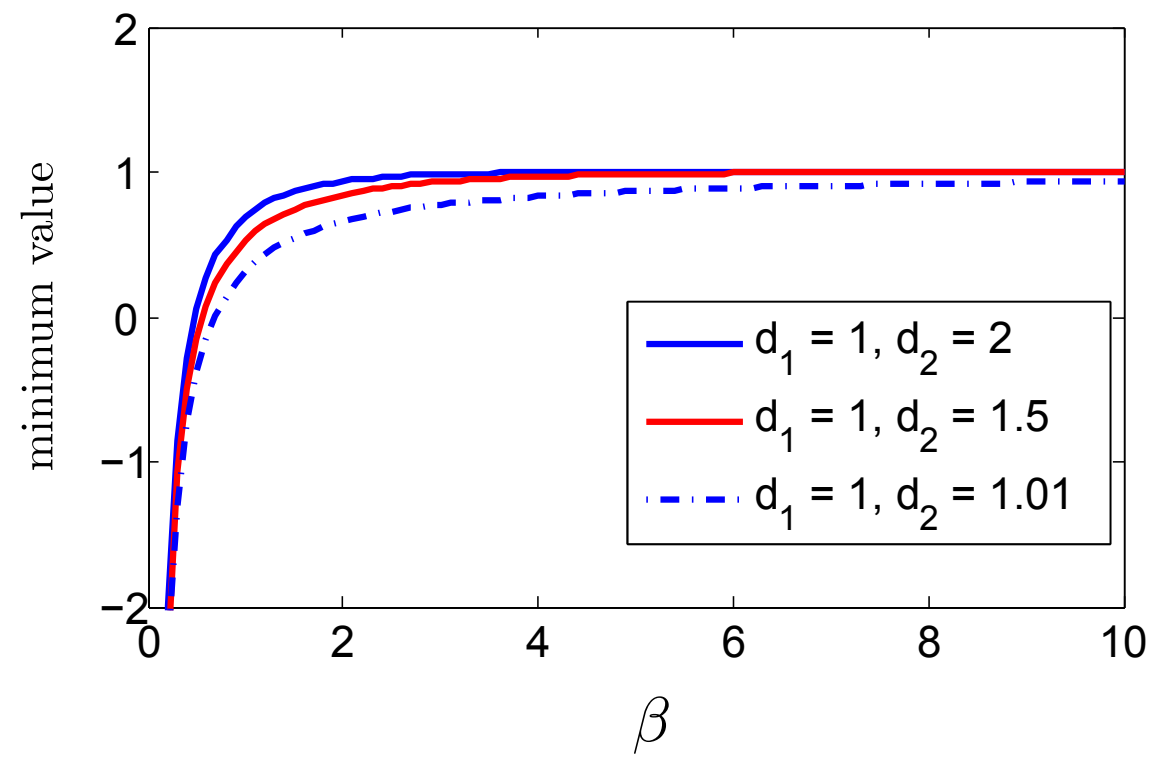

Figure 3.1: Approximate minimum distance versus $\beta$ for three sets of two distances

the smallest $d_{i}$.

Figure 3.1 provides an example of how the approximation is affected by $\beta$ for three sets of two distances. In each set, the minimum distance is unity. Notice that the approximation becomes less accurate for smaller $\beta$ as the difference between the distances approach one another.

Applying (3.17) to (3.16), the new objective is,

$$
\arg \max _{\phi_{\mathbf{i}}}-\ln \left(\sum_{i \neq j} \exp \left(-\beta\left\|\phi_{\mathbf{i}}-\phi_{\mathbf{j}}\right\|_{2}\right)\right) / \beta
$$

The problem is now differentiable, although non-convex, with convex inequality constraints and affine equality constraints. 


\subsection{MATLAB fmincon}

Find minimum of constrained nonlinear multi-variable function (fmincon) is a MATLAB function that solves optimization problems of the form,

$$
\begin{array}{ll}
\underset{x}{\operatorname{minimize}} & f_{o}(x) \\
\text { subject to } & A x \leq b \quad C x=d \quad g(x) \leq 0 \quad k(x)=0
\end{array}
$$

where $f_{o}(x), g(x)$ and $k(x)$ may be non-linear, potentially non-convex, functions that must be continuous and have continuous first derivatives. As all the constraint's described by (3.11) and (3.15) are linear and expressed in matrix form, the constraints involving $g(x)$ and $k(x)$ are inactive. When invoked, fmincon attempts to find a constrained minimum of a scalar function of several variables starting at an initial estimate. The fmincon function uses one of four algorithms, as chosen by the user, to carry out the optimization process. The default algorithm, and the one chosen for this work, is the interior point algorithm.

Interior point methods are a class of optimization algorithms that solve a problem with linear equality and inequality constraints by reducing it to a sequence of linear equality constrained problems $[83,84]$. The sequence of new problems is easily solved by the fmincon optimization algorithms; however they require the derivation of the Hessian of the objective, hence the requirement for $f_{o}$ to be twice differentiable. The interior point method was selected as is is a large scale algorithm. An optimization algorithm is considered large scale when it uses sparse linear algebra that does not need to store, nor operate on, full matrices [84]. As a result, large scale algorithms handle large, sparse problems, efficiently while still being able operate on small dense 
problems. The downside to large scale algorithms is that they limit the type of constraints the algorithm can operate over; however, as seen in (3.11) and (3.15), the only constraints are sparse linear inequalities and affine equalities.

\subsubsection{Stopping Conditions}

The following is the output of the fmincon interior point algorithm at the end of the optimization process described in the next section, and will be referred to as a Type1 termination,

Local minimum found that satisfies the constraints.

Optimization completed because the objective function is non-decreasing in feasible directions, to within the default value of the function tolerance, and constraints are satisfied to within the default value of the constraint tolerance.

The relative first-order optimality measure, $5.404733 \times 10^{-7}$, is less than options. TolFun $=1.000000 \times 10^{-6}$, and the relative maximum constraint violation, $0.000000 \times 10^{0}$, is less than options. TolCon $=1.000000 \times 10^{-6}$.

The output signifies that fmincon has located a local minimum that satisfies the design constraints. The constraint tolerance, TolCon, is an upper bound on the magnitude of all constraint functions and is used by the solver to identify when the argument violates the constraints. Violation of TolCon will not cause the solver to stop. The stopping conditions are based on the function tolerance, TolFun, which 
is a lower bound on the change in the value of the objective function during a step. If $\left|f_{o}\left(x_{i}\right)-f_{o}\left(x_{i+1}\right)\right|<$ TolFun, where $x_{i}$ and $x_{i+1}$ are the argument at iterations $i$ and $i+1$ respectively, the iterations end. TolFun is also a bound on the first-order optimality measure. If the optimality measure is less than TolFun, the iterations end. The definition of the first order optimality conditions is based on the Karush-KuhnTucker (KKT) conditions. As the KKT conditions are only necessary conditions for optimality, as local maxima and saddle points also satisfy them, the solver also specifies that the objective is non-decreasing in all feasible directions, which signifies the function is locally convex in all directions. A feasible direction is any direction vector that when added to the current feasible point produces another feasible point.

At some stages during the optimization process described in the next section, it is possible for the solver to return with what will be referred to as a Type2 termination,

Local minimum possible. Constraints satisfied.

fmincon stopped because the size of the current step is less than the default value of the step size tolerance and constraints are satisfied to within the default value of the constraint tolerance.

Optimization stopped because the relative changes in all elements of $\mathrm{x}$ are less than options.TolX $=1.000000 \times 10^{-10}$, and the relative maximum constraint violation, $0.000000 \times 10^{0}$, is less than options.TolCon $=$ $1.000000 \times 10^{-6}$.

The step tolerance, TolX, is a lower bound on the size of a step, $\left|x_{i}-x_{i+1}\right|$. If the 
solver attempts to take a step that is smaller than TolX, the iterations end. In this case, the first order optimality may not be met, but the values of the argument are not changing with each iteration signifying a local minimum is possible.

\subsubsection{Starting Point}

Interior point methods require a strictly feasible point, $\mathbf{A x}<\mathbf{b}$, to act as a starting point for the algorithm. Strict feasibility is required as iterations of interior point methods approach the optimal solution by traversing the interior space of the constraint region. The fmincon starts by finding a strictly feasible point based an an

initial guess, $\overline{\phi_{\text {int }}}$, provided by the calling function, which may or may not be strictly feasible. As discussed in Section 3.2, the objective is non-convex; therefore, the local minima found by fmincon depends on $\overline{\phi_{\text {int }}}$. In order to find the best possible local minima, the optimization process must be carried from multiple starting points. In this work, each starting point was randomly chosen from a uniform probability distribution.

\subsection{Constellation design using fmincon}

Using the continuous approximation in (3.18), and the standard form for the constraints in (3.11) and (3.15), the problem can be directly solved by fmincon. However, when the argument approaches a local optima, the smallest distances between all symbols approach the same value. As seen previously in Figure 3.1, this will cause (3.18) to produce poor results for small values of $\beta$. The result can be improved by choosing an arbitrarily large $\beta$ value. However, a large $\beta$ will produce large gradients 
when finding the Hessian, causing the algorithm to either converge slowly or not at all. A good approach to obtain a reliable approximation without poor convergence is to apply the interior point method to the problem multiple times, starting with a small $\beta$ and increasing $\beta$ in each iteration [82]. With each successive $\beta$ the optimal solution of the previous $\beta$ acts as the starting point for the next. This process is summarized by,

$$
\begin{aligned}
& \phi_{i} \leftarrow \text { random starting point } \\
& \beta \leftarrow 1 \\
& \text { while error expression do } \\
& \phi_{\text {opt }} \leftarrow \arg \max _{\phi_{\mathbf{i}}}-\ln \left(\sum_{i \neq j} \exp \left(-\beta\left\|\phi_{\mathbf{i}}-\phi_{\mathbf{j}}\right\|_{2}^{2}\right)\right) / \beta \\
& \quad \beta \leftarrow 2 \beta \\
& \phi_{i} \leftarrow \phi_{\text {opt }}
\end{aligned}
$$

\section{end while}

The process of doubling beta continues until the mean squared difference between each symbol of the current and past iterations optimal argument is less than a specified threshold, typically $1 e-8$. The termination criteria for a given iteration of $\beta$ is as discussed in Section 3.3.1. Small $\beta$ values may cause the solver to terminate with Type2 locally optimal solutions; however, as $\beta$ gets larger, the solver terminates exclusively with Type1 optimal solutions as the solver has had more time to converge on a local optima. 


\subsection{Design Samples}

In this section, constellations found through the proposed method are presented. For all figures, the triangle defines the normalized design space, the cross represents the color balance point in the normalized design space and the circles each have a diameter equal to the minimum distance between all symbols in the constellation. All design samples were taken as the best solution over forty random starting points and the numeric values each sample can be found in Appendix A.

Consider an 8-CSK constellation without a specified operating chromaticity, normalized operating intensity $L=1$, equally likely symbols, an ideal (identity) channel matrix, and all peak power constraints $L_{r, g, b}>L$. Using the transform defined by equation (3.4), the normalized design space is represented as an equilateral triangle. This scenario is of interest, as it is equivalent to packing eight equally sized disks in an equilateral triangle, a problem with a known analytic global optimum [85].

Figure 3.2a shows the best result for this scenario achieved using the method described in Section 3.4. The result in Figure 3.2a coincides with the known optimal disk packing solution and achieves a minimum distance of 0.4848 in the normalized design space [85] . By adjusting the $\beta$ parameter, the reported result can achieve a relative error of up to $10^{-12}$ of the global optimum [85]. This result also agrees with the earlier result reported by Drost et. al [47]. An interesting observation about this result is that it also coincides with 8-CSK design rule outlined in IEEE 802.15.7.

Figure $3.2 \mathrm{~b}$ shows an 8-CSK design considering a color balance at the centroid of the normalized design space. The minimum distance in the normalized design space is 0.4714 , marginally worse than the case with no color balance. Coincidentally, the designed constellation corresponds to the optimal packing of ten disks in an equilateral 


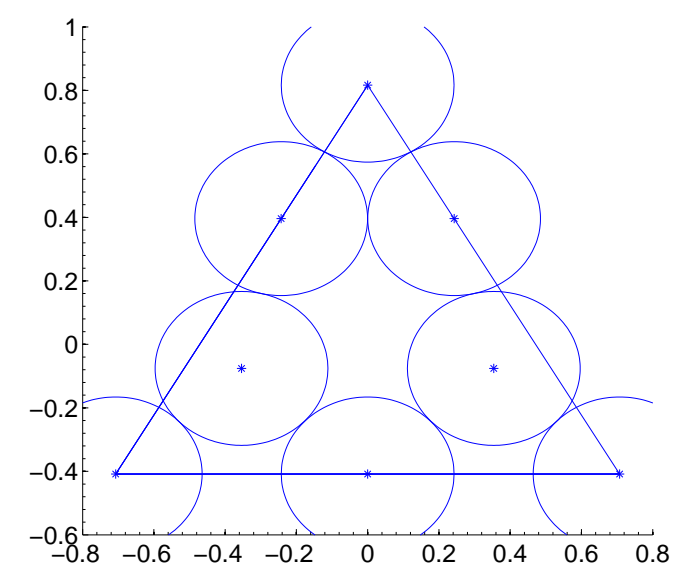

(a) No color balance

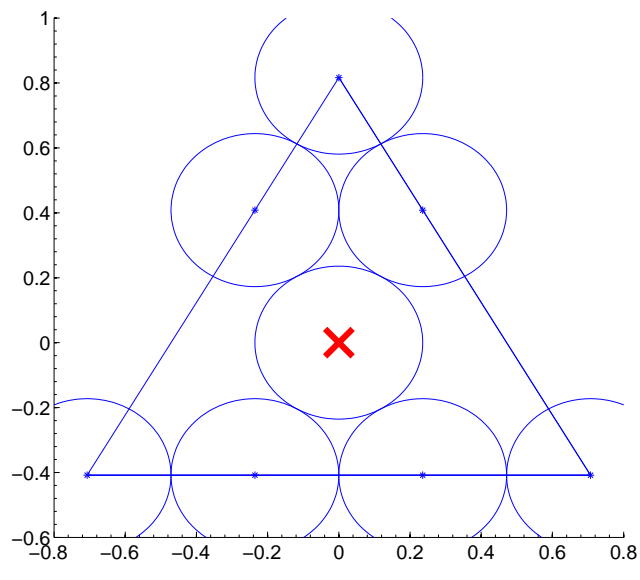

(b) $\mathbf{d}^{*}=[0,0]$

Figure 3.2: 8-CSK with and without specified color balance. $L=1, L_{r, g, b}>L$ and identity channel matrix.

triangle with two disks removed [85].

Figure 3.3 shows 32-CSK with color balanced away from the centroid, and color balanced at the centroid of the normalized design space. The minimum distances in the normalized design space for the two scenarios are 0.1569 and 0.2025 respectively. This result clearly shows that if the specified operating chromaticity moves the color balance point in the normalized design space away from the centroid, the minimum distance is reduced leading to a higher SER. While the optimality of these results cannot be demonstrated analytically, the dense near hexagonal packing of the solutions, which efficiently place points within the design constraints, suggest a local minima.

Figure 3.4 presents a 16-CSK design with peak intensity constraints $L_{r}=0.8 \mathrm{~L}$ and $L_{g}=L_{b} \geq L$, and compares it to the case of $L_{r}=L_{b}=L_{g} \geq L$. Both scenarios assume an ideal channel matrix, and a color balance at the centroid of the normalized design space. The minimum distances in the normalized design space are 0.2915 and 


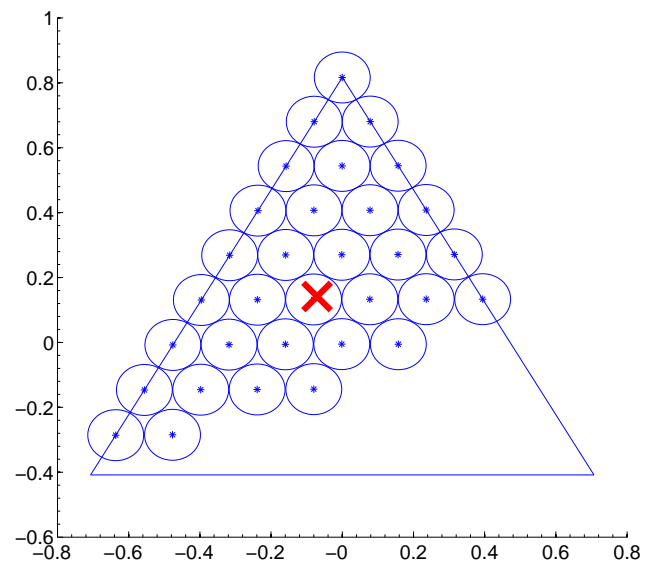

(a) $\mathbf{d}^{*}=[-0.1, .2]$

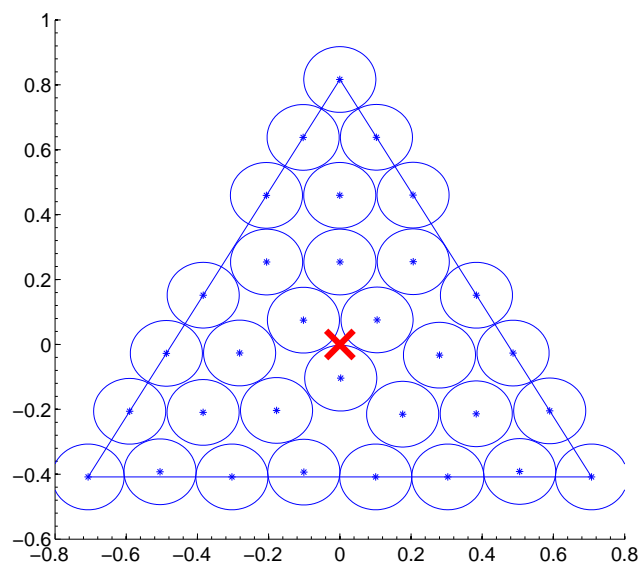

(b) $\mathbf{d}^{*}=[0,0]$

Figure 3.3: 32-CSK for two different color balance points. Both cases have identity channel matrix, with $L_{r, g, b} \geq L$

0.3021 respectively. Therefore, for a given operating intensity, truncation reduces the achievable minimum distance of the design. Again, no comment can be made on the optimality of the solution, but dense and regular packing of the constellation points suggests local minima were identified.

Next, consider a case with cross-talk between the color channels due to an overlap in the emission spectrum of the three colored LEDs and/or the transmission spectrum of the receiver optical filters. Let $L_{r, g, b} \geq L$ and assume that all symbols are equally likely with a operating chromaticity such that $\mathbf{d} *=[\mathbf{0}, \mathbf{0}]$. Let the channel matrix representing the channel cross talk be as in [47]

$$
\mathbf{H}=\left[\begin{array}{ccc}
1 & 0 & 0 \\
0 & 0.8 & 0.1 \\
0 & 0.1 & 0.8
\end{array}\right]
$$




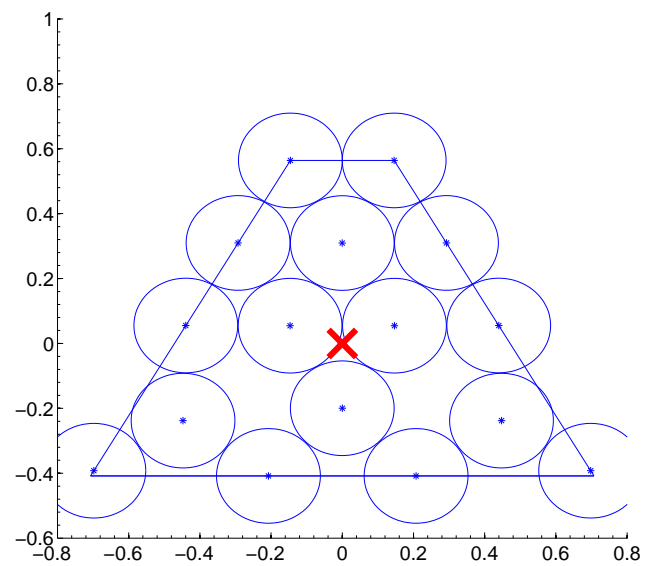

(a) $\mathbf{d}^{*}=[0,0], L_{g, b} \geq L$, and $L_{r}=0.8$

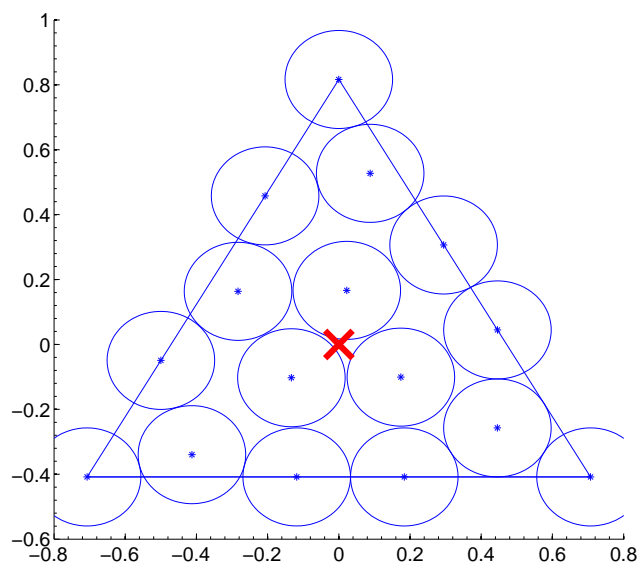

(b) $\mathbf{d}^{*}=[0,0], L_{r, g, b} \geq L$

Figure 3.4: 16-CSK with and without dimension truncation, including color balancing. Both cases have identity channel matrix

Figure 3.5 (a) shows the signal set, $\phi_{i}$, designed in the normalized design region. The corresponding transmitter constellation, $\mathbf{s}_{\mathbf{i}}$ is shown in Figure 3.5 (b) . The minimum distance of this constellation in the normalized design space is 0.2474 . Notice again that a dense, near hexagonal, packing in the normalized design space is achieved.

\subsection{Hexagonal Lattice Heuristic}

While the method proposed in Section 3.4 is capable of managing large constellations, those in excess of 128 symbols can take extended periods of time to simulate. Operating MATLAB in real-time on a Windows XP machine with a $3.2 \mathrm{GHz}$ processor, 128-CSK took approximately 2 hours for a single starting point. However, large constellations may not require the precision of high end convex optimization. Figure 3.6 shows results for 128-CSK with equally likely symbols and no color balance. Notice 


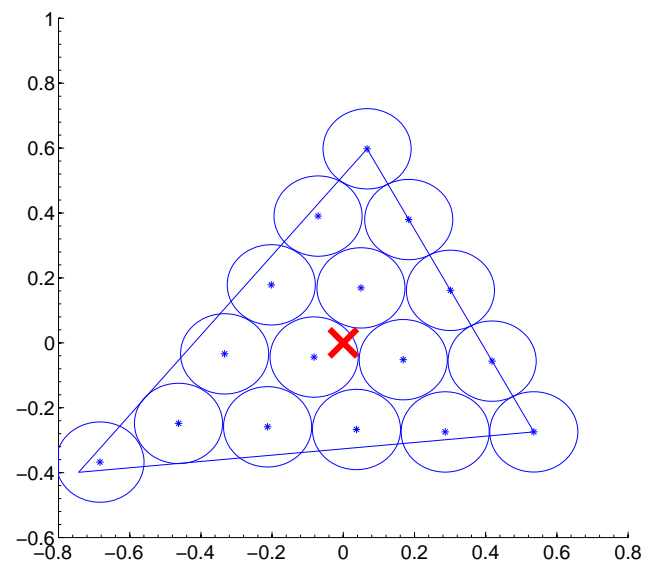

(a) Optimal set $\phi_{i}$

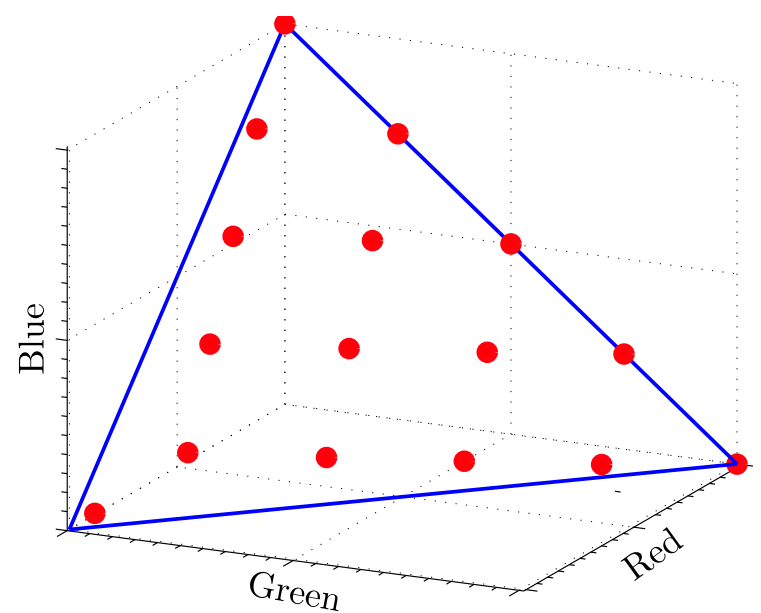

(b) Transmit set $\mathbf{s}_{\mathbf{i}}$

Figure 3.5: Optimal 16-CSK alphabet, in both the normalized design space and transmitted intensity coordinates, for $L=1, L_{r, g, b} \geq L$, and non-ideal invertible channel matrix

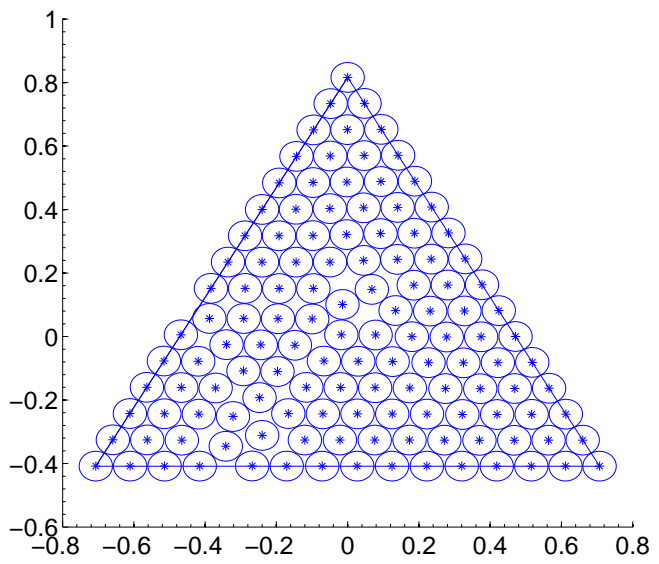

(a) Identity channel matrix

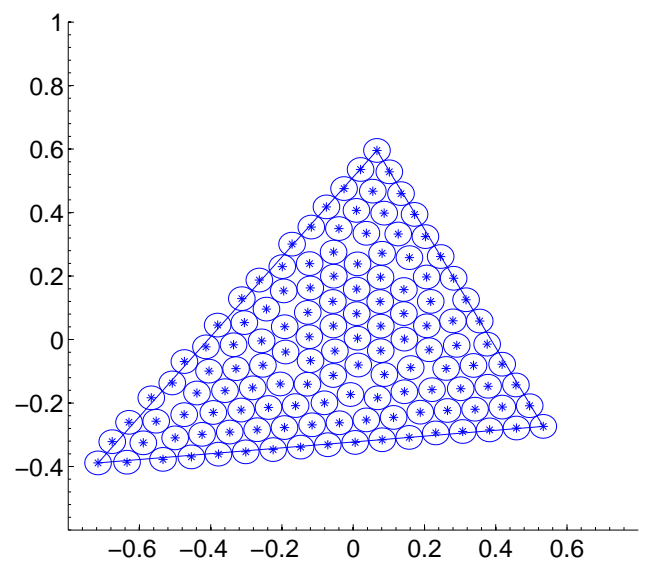

(b) Non ideal channel matrix

Figure 3.6: 128-CSK, $L_{r, g, b} \geq L$ with no color balance for both the identity channel matrix and the channel matrix specified by equation (3.19) 
that the results both exhibit near hexagonal packing. These results suggest that intersecting a hexagonal lattice code with the normalized design space may provide an adequate signal alphabet when the operating chromaticity need not be specified.

Figure 3.7 shows the normalized minimum distance versus constellation size for no color balance using this heuristic compared against the convex optimization technique. The approximation only provides marginally decreased minimum distances for small signal sets, and as the number of points in the system increases, the minimum distances of constellations produce by the heuristic approaches that of the original proposed method. Specifically, consider the constellations shown Figure 3.6, which were designed using the method proposed in Section 3.4, and compare them to the constellations shown in Figure 3.8, which were designed under the same constraints using the heuristic. The minimum distances in Figure 3.6a and Figure 3.6a are 0.0905 and 0.0747 respectively. The minimum distances for the same constellations designed with heuristic are 0.0870 and 0.0735 , which have a relative difference of only 3.87 $\%$ and $1.61 \%$ respectively. Furthermore, the heuristic took 0.253 seconds, on the same machine, to produce the depicted results compared to the two hours, per starting point, using convex optimization. While the heuristic is efficient, it is limited to inequality constrained problems only and therefore cannot optimize for a specific operating chromaticity.

\subsubsection{The Heuristic Optimization Process}

The algorithm for the heuristic contains two stages of optimization consisting of a line search and a bisection search. A hexagonal grid, aligned with one side of normalized design space, is generated such that it fills the box that bounds all vertices of the 


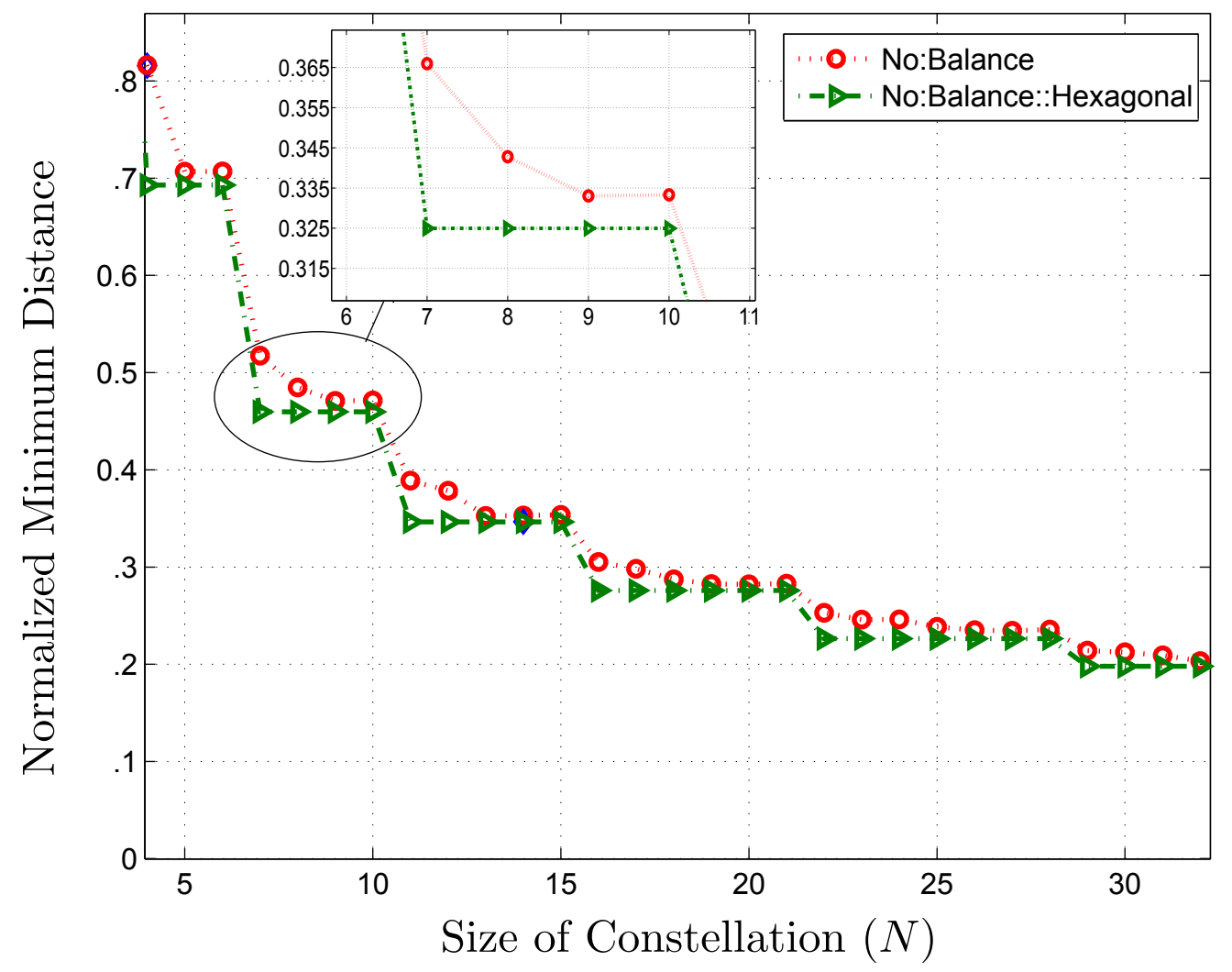

Figure 3.7: Minimum distance, in the normalized design space, versus constellation size for $L_{r}=L_{b}=L_{g} \geq L$, no color balance, and $\mathbf{H}$ is the identity matrix 


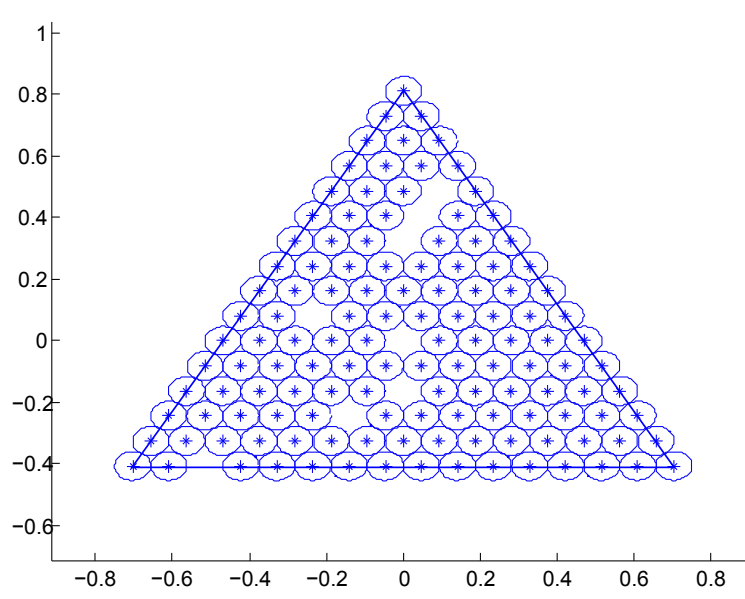

(a) Identity channel matrix

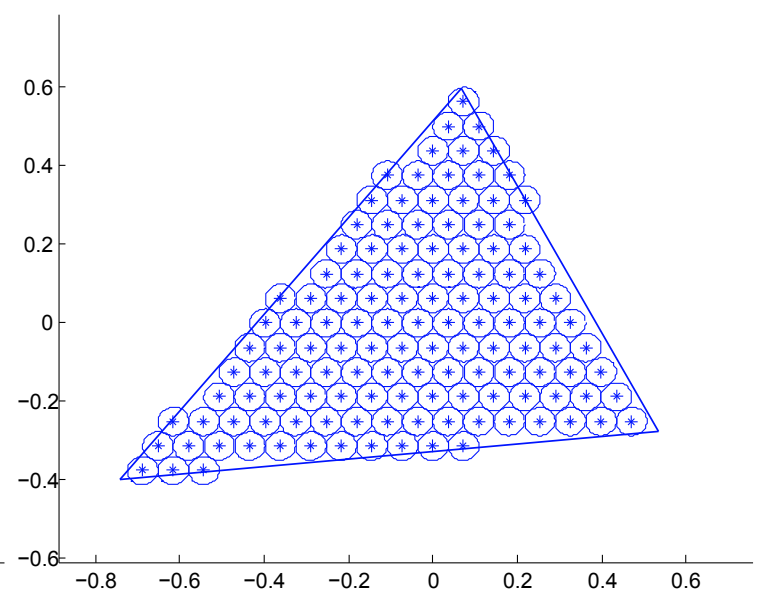

(b) Non ideal channel matrix

Figure 3.8: 128-CSK, achieved with the lattice heuristic, for $L_{r, g, b} \geq L$ with no color balance for both the identity channel matrix and the channel matrix specified by equation (3.19)

normalized design space. The initial minimum distance between grid point is chosen such that at least $N$ points lie inside the polygon. The heuristic then counts the number of points inside the polygon and scales the minimum distance of the lattice by a fixed step. This process continues until at there are less than $N$ points inside the region. Therefore, in the current iteration, less than $N$ points are bounded, and in the previous iteration, greater than $N$ points are bounded. The optimal solution for the heuristic is then bounded between the minimum distances that produced these two points.

To reduce the computation time required for the subsequent optimization, all points outside the bounded region for the case with larger than $N$ bounded points are removed. The next step of the optimization is to scale the remaining lattice points via bisection search to find the largest lattice spacing without causing the number of points bounded to drop below $N$. This process continues until the mean squared difference between the current and previous lattice spacing is less than a specified 
tolerance. As the intersection of a lattice with the space does not generate exactly $N$ points, constellations of similar size will have the same number of points bounded in the region and therefore have the minimum distance. Points in excess of $N$ are simply thrown away at the discretion of the designer. This behavior is the reason for the step like structure of the minimum distance versus constellation size, when using the heuristic, seen previously in Figure 3.7.

\subsection{Constraint Relaxation}

As discussed in Section 2.5.5, CSK is intended to operate with a constant luminous flux to mitigate flicker. However, the discussion in Section 2.1.3 revealed that the average observer cannot perceive flicker at high frequencies. Section 3.1.3 discussed modifying the CSK constraints to design constellations that operate with a fixed current in place of a fixed luminous flux in order to reap the benefits of a static current draw. However, optimizing for either constant current or constant luminous flux reduces the degrees of freedom available to maximize the SER of the system for a given rate. Instead of fixing the luminous flux or current, the constraints can be relaxed in favor of constraining the maximum allowable ripple on either the output luminous flux or input electrical current. As long as the some ripple is tolerable, the minimum distances of designed constellations can be improved, thus reducing the SER at a given SNR. The relaxation is referred to as 3-D CSK for the remainder of this work. Furthermore, the numeric values of each design sample presented can be found in Appendix A. 


\subsubsection{Optimization Problem}

The 3D-CSK optimization problem operates on the intensity component symbol, $\mathbf{s}_{\mathbf{i}}$, and is given as,

$$
\begin{array}{ll}
\underset{\mathbf{s i}_{\mathbf{i}}, t}{\arg \max } & \min _{i \neq j}\left\|\mathbf{H}\left(\mathbf{s}_{\mathbf{i}}-\mathbf{s}_{\mathbf{j}}\right)\right\|_{2} \\
\text { subject to } & L_{\text {min }} \leq[111] \cdot \mathbf{s}_{\mathbf{i}} \leq L_{\text {max }}, i=1 \ldots m \\
& 0 \leq l_{r, g, b} \leq L_{r, g, b} \\
& \frac{1}{N} \sum \mathbf{s}_{\mathbf{i}}=t \mathbf{d}
\end{array}
$$

where $L_{\max }$ and $L_{\min }$ are the maximum total luminous flux and minimum total luminous flux, $t \mathbf{d}$ represents the set of points that produce the required operating chromaticity and $\mathbf{d}$ is as defined previously in (3.12). Recall from (2.11) that $\mathbf{d}$ is related to the $X Y Z$ tristimulus of each colored LED. Furthermore, recall from (2.8) that the $X Y Z$ tristimulus are directly proportional to the SPDs of each LED. Therefore, if the luminous flux, $Y$ tristimulus, of an LED where to scale by $t$, then $X$ and $Z$ must also scale by $t$. As the $x y$ chromaticity coordinates are invariant to scale, it follows that the set of points that produce a given chromaticity must be a straight line when changing the average luminous flux.

When the peak constraints on each individual channel are not active, the constraint region is the intersection of two parallel planes with the positive orthant in $\mathbb{R}_{+}^{3}$, where the distance between the two planes represents the allowable ripple. If a color channels peak constraint is active, then the constraint region is defined as before with an additional intersecting plane normal to the corresponding color channels intensity axis. 
As with the constant luminous flux CSK model, the objective is both non-convex and non-differentiable. Therefore, the continuous approximation defined in (3.17) must be applied to the objective in (3.7.1), to produce the objective,

$$
\arg \max _{\mathbf{s}_{\mathbf{i}}}-\ln \left(\sum_{i \neq j} \exp \left(-\beta\left\|\mathbf{H}\left(\mathbf{s}_{\mathbf{i}}-\mathbf{s}_{\mathbf{j}}\right)\right\|_{2}\right)\right) / \beta
$$

Furthermore, in order to allow the problem to be solved by fmincon, the constraints must be expressed in a standard matrix form. Expressing the constraints for 3D CSK in matrix form is mathematically simpler when compared to the constant flux/current case as they do not depend on $\mathbf{H}$, and the use of the orthographic projection is not required. The inequality constraints are expressed by,

$$
\overline{\mathbf{A}_{3 \mathbf{D}}}\left[\begin{array}{c}
\overline{\mathbf{s}} \\
t
\end{array}\right]=\left[\begin{array}{ccccc}
\mathbf{A}_{3 \mathrm{D}} & 0 & 0 & 0 & 0 \\
0 & \mathbf{A}_{3 \mathrm{D}} & 0 & 0 & 0 \\
0 & 0 & \ddots & 0 & 0 \\
0 & 0 & 0 & \mathbf{A}_{3 \mathrm{D}} & 0 \\
0 & 0 & 0 & 0 & -1
\end{array}\right]\left[\begin{array}{c}
\overline{\mathbf{s}} \\
t
\end{array}\right] \leq \overline{\mathbf{b}_{3 \mathbf{D}}}=\left[\begin{array}{c}
\mathbf{b}_{3 \mathrm{D}} \\
\mathbf{b}_{3 \mathrm{D}} \\
\vdots \\
\mathbf{b}_{3 \mathrm{D}} \\
0
\end{array}\right]
$$

where $\overline{\mathbf{s}}$ is the column wise vectorization of $\mathbf{s}_{\mathbf{i}}$, and

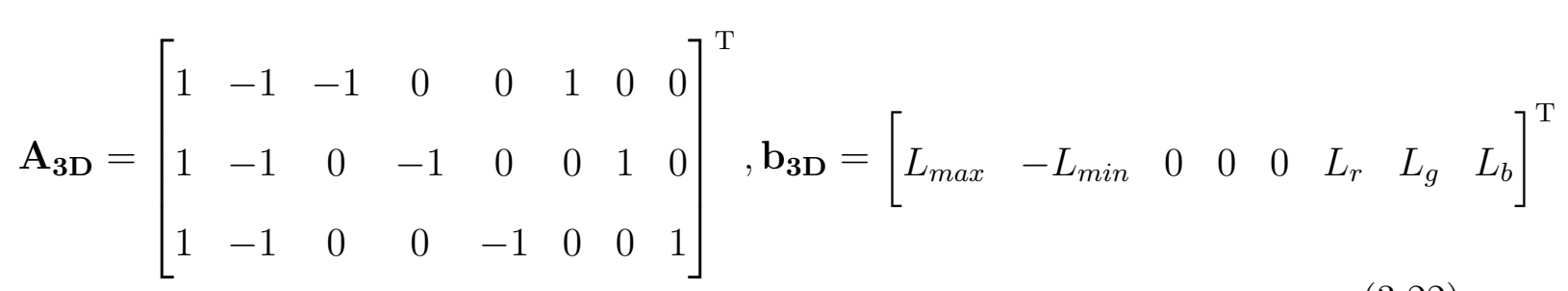

where $\mathrm{T}$ denotes matrix transpose. 


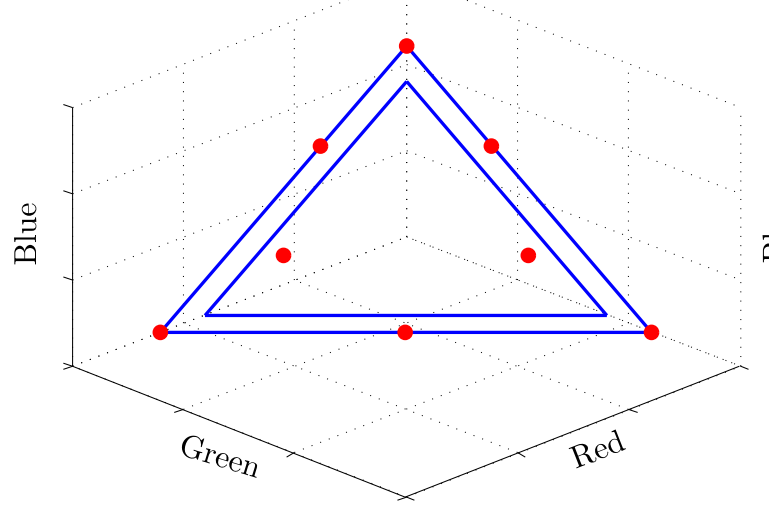

(a) Transmit set

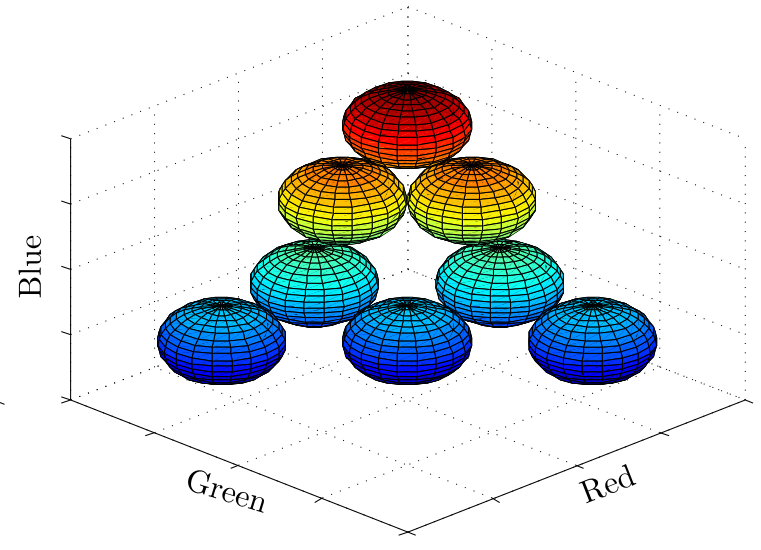

(b) Receive set

Figure 3.9: Depiction of 3D 8-CSK constellation as seen by both the transmitter and receiver. Channel matrix is identity, no operating color is specified, $L_{\max }=1.1$ and $L_{\min }=0.9$.

The equality constraint is expressed by,

$$
\left[\begin{array}{ll}
\mathbf{C}_{3 \mathrm{D}} & -\mathbf{d}
\end{array}\right]\left[\begin{array}{l}
\overline{\mathrm{s}} \\
t
\end{array}\right]=\mathbf{0}
$$

where $\mathbf{C}_{3 \mathbf{D}}$ is formed by the $N$ fold horizontal replication of the $3 \times 3$ identity matrix normalized by $N$.

\subsubsection{Design Samples}

All design samples shown in this section were taken as the best solution over forty random starting points. The diameter of the spheres in each figure represent the minimum distance between points, as seen by the receiver.

Figure 3.9 depicts an 3D 8-CSK constellation, including a depiction of the constraint region, for an identity channel matrix, no specified operating chromaticity, $L_{\max }=1.1$ and $L_{\min }=0.9$, and no independent peak constraints on a color channel. 


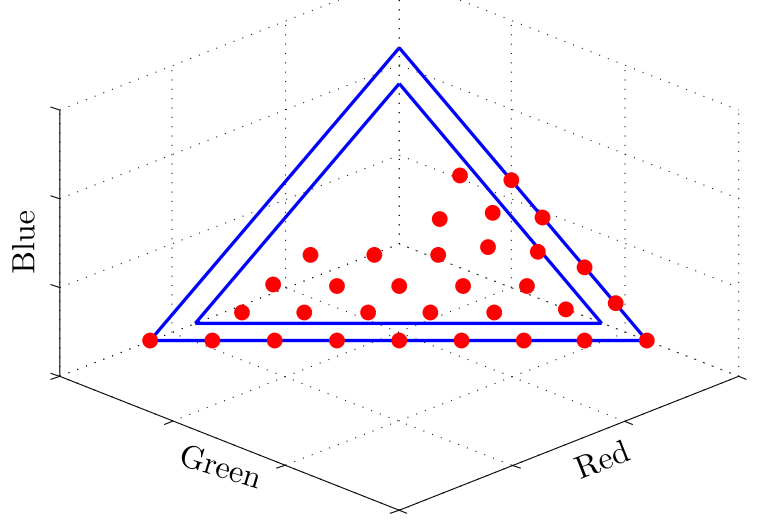

(a) Transmit set

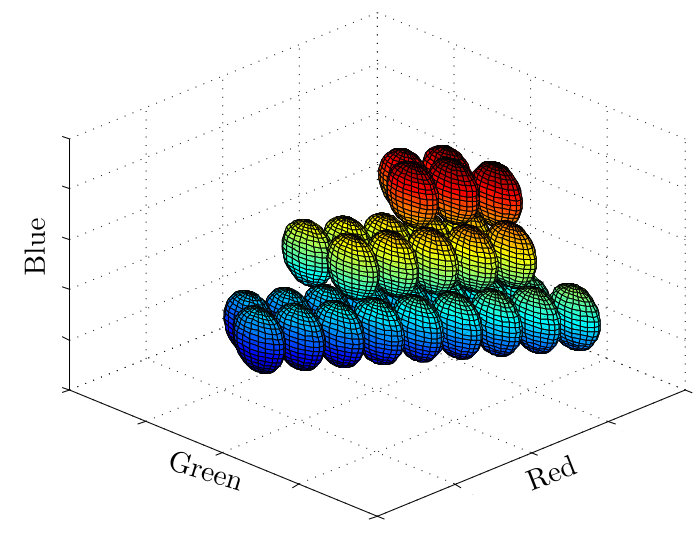

(b) Receive set

Figure 3.10: Depiction of 3D 32-CSK constellation as seen by both the transmitter and receiver. Channel matrix is identity, $L_{\max }=1$ and $L_{\min }=0.1$, no independent peak constraints on a color channel, and with an operating chromaticity corresponding to $\mathbf{d}=[0.3443,0.4857,0.1700]$

The result in Figure 3.9 (b) appears remarkably similar to the 8-CSK example shown previously in Figure 3.2, which consequently was the 8-CSK constellation proposed by IEEE 802.5 .7 ; however the $\pm 10 \%$ ripple allowance produced a $12.2 \%$ improvement in minimum distance after normalizing the two results to have the same average luminous flux. As with the samples discussed in Section 3.5, no comment can be made on the global optimality of this design. However, the example does demonstrate that even small relaxations in the constant luminous flux constraint can produce significant improvements in the minimum distance between CSK symbols.

The result in Figure 3.10 depicts a 3D 32-CSK constellation for an identity channel matrix, $L_{\max }=1.1$ and $L_{\min }=0.9$, no independent peak constraints on a color channel, and with an operating chromaticity corresponding to $\mathbf{d}=[0.3443,0.4857,0.1700]$. The $\mathbf{d}^{*}$ corresponding to the aforementioned $\mathbf{d}$ is identical to that specified in Figure 


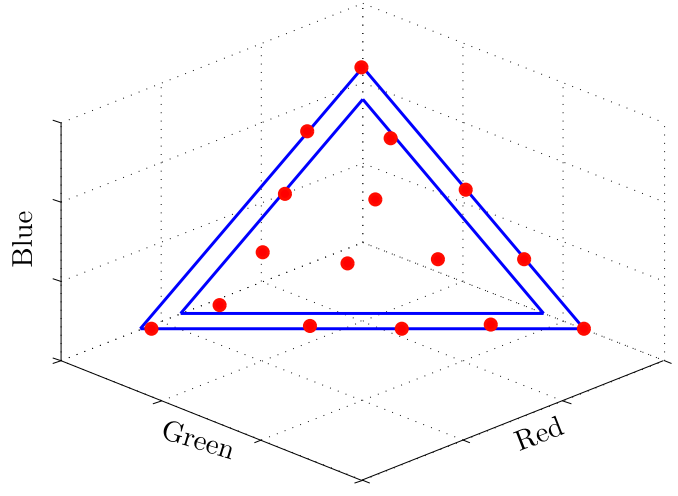

(a) Transmit set

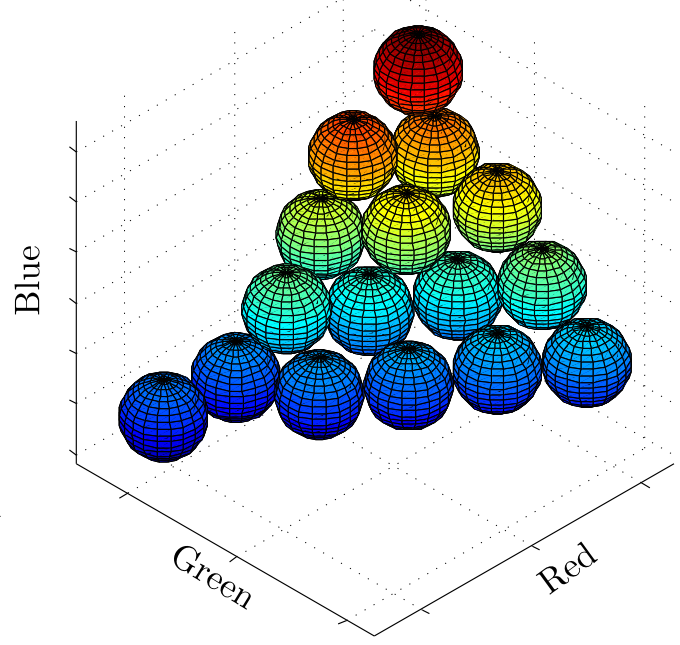

(b) Receive set

Figure 3.11: Depiction of 3D 16-CSK constellation as seen by both the transmitter and receiver. Channel matrix be as in equation (3.19), $L_{\max }=1.1$ and $L_{\min }=0.9$ and $\mathbf{d}=[0.2883,0.3203,0.3203]$

3.3a. The minimum distance of this constellation, when operating at the same average luminous flux of the constant luminous flux case, is 0.1942 compared to 0.1569 representing an $23.7824 \%$ improvement. While no comment can be made on the optimality of this result, the constellation does satisfy all the constraints, and the dense packing of the structure suggests the solution is efficiently distributing the constellation points within the constraint region.

The final example shown in Figure 3.11 is provided to demonstrate that the algorithm is capable of optimizing constellations when crosstalk exists between the color channels. The channel matrix is as in (3.19), $L_{\max }=1.1$ and $L_{\min }=0.9$ and $\mathbf{d}=[0.2883,0.3203,0.3203]$ is selected such that $\mathbf{d}^{*}=[0,0]$, as it in the example shown in Figure 3.5. After normalizing the result to have the same average luminous flux, the 3D CSK constellation shows an $16.695 \%$ improvement in minimum distance, 
from 0.2474 to 0.2887 , by allowing a $\pm 10 \%$ ripple.

\subsection{Conclusions}

In this chapter, a differentiable approximation to the CSK constellation design problem for arbitrarily sized constellations was demonstrated. The approach was shown to be compatible with any convex polygon constraint regions, and subsequently any invertible linear transform on the constraint region, such as the CSK channel gain matrix. Furthermore, a modification to the approach was demonstrated to be able to design three dimensional CSK constellations that had improved minimum distance. Additionally, a simple heuristic was demonstrated for deriving constellations for large symbol sets when considering constant luminous flux or constant electrical current constellations with no specified operating chromaticity. While the heuristic cannot specify the chromaticity of the source, its computation time was shown to be substantially shorter than that of the interior point methods for constellations of the same size.

This chapter also provided various design samples that demonstrated the negative impacts of peak intensity constraints on individual color channels, such as those imposed by peak current limitations, on the achievable minimum distance of the design. Furthermore, the convex optimization methods proposed were demonstrated to allow the chromaticity of the source to be specified. It was observed that if a desired operating chromaticity placed a mean constraint, on the constellation, away from the center of the constraint region that the achievable minimum distance would be reduced. 
In the following chapter, a CSK communication channel is implemented and characterized. The channel will act as a test bed to compare the SER versus SNR and illuminance of the IEEE 802.15.7 constellations against various constellations designed using the methods for constant current constellations discussed in this chapter. As no 3D CSK constellations exist in literature to act as a bench mark, and the SER is directly related to minimum distance, 3D constellations, and constellations designed with the heuristic, are not considered in subsequent chapters. 


\section{Chapter 4}

\section{Implementation and}

\section{Characterization of an}

\section{Experimental CSK Compatible}

\section{Communication System}

In this chapter, the implementation and characterization of a CSK compatible VLC link is discussed. Characterization includes analysis of the system noise, bandwidth, linearity, photo response versus illumination, and channel gain matrix. Furthermore, this chapter provides detail on relating the measured illuminance, a quantity that is standardized in the lighting industry, to the measured SNR of the implemented CSK compatible VLC link. 


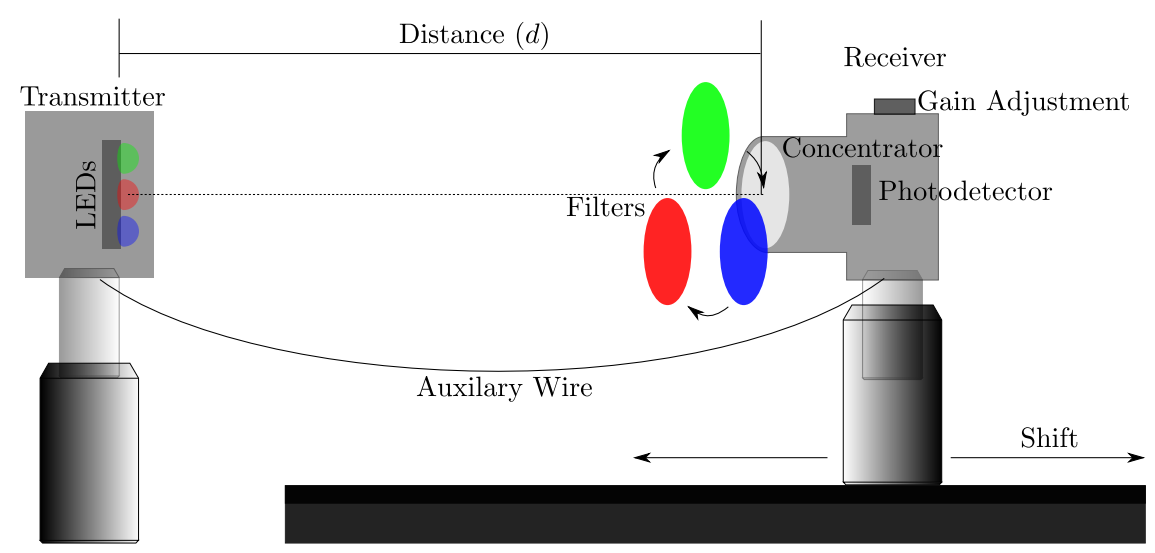

Figure 4.1: CSK experimental test bed

\subsection{Implementation Details}

Figure 4.1 depicts the experimental test bed that was used for measuring the error performance of CSK constellations. The transmitter was mounted on a stationary optical post with the center of the luminary aligned with the center of the receiver aperture. The receiver was mounted on a rail in order to allow it to slide freely in one direction to adjust distance. Additionally, an auxiliary wire was placed between the transmitter and receiver for synchronization purposes only. As only one photoelement was used, capturing all three color bands required that all three colors be transmitted simultaneously while the receiver independently captured each channel by cycling between the red, green, and blue filters. In order to synchronize the three independent color measurements, a predefined data sequence known to the receiver was used in conjunction with the auxiliary wire. 


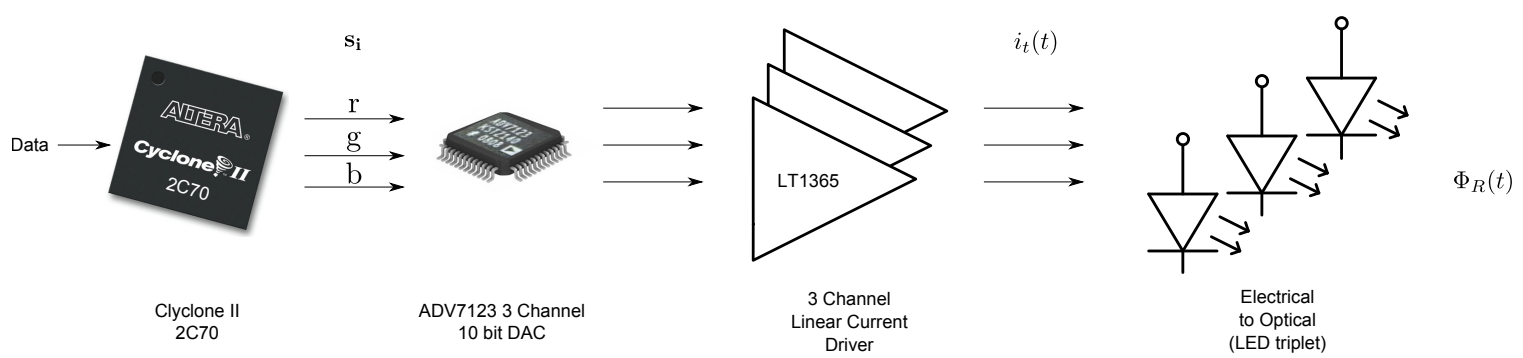

Figure 4.2: Functional block diagram of transmitter

\subsubsection{Transmitter Elements}

Figure 4.2 and Figure 4.3 respectively depict a functional block diagram and photograph of the test beds transmitter. The transmitter consisted of an FPGA for data modulation, a three channel digital-to-analog converter, three custom high power linear current drivers and three colored LEDs (red, green, and blue).

\section{Modulation and Digital to Analog Conversion}

The Altera DE2-70 FPGA development board [86] was used to modulate and convert data into analog CSK signals for transmission. The DE2-70 development board was selected as it is outfitted with the Analog Devices ADV7123 video graphics array (VGA) DAC [87]. The VGA interface of the ADV7123 is ideal for CSK as it has three independent, 10 bit channels, that can be used to control the three colored LEDs simultaneously. For each constellation, the required DAC levels were stored in the Cyclone II 2C70 FPGA [88] on-chip ROMS. During transmission, symbols were loaded onto the DAC input bus, during an edge transition of the symbol clock, depending on the data source. The symbol clock was generated via clock division of the $50 \mathrm{MHz}$ oscillator of the DE2-70. 


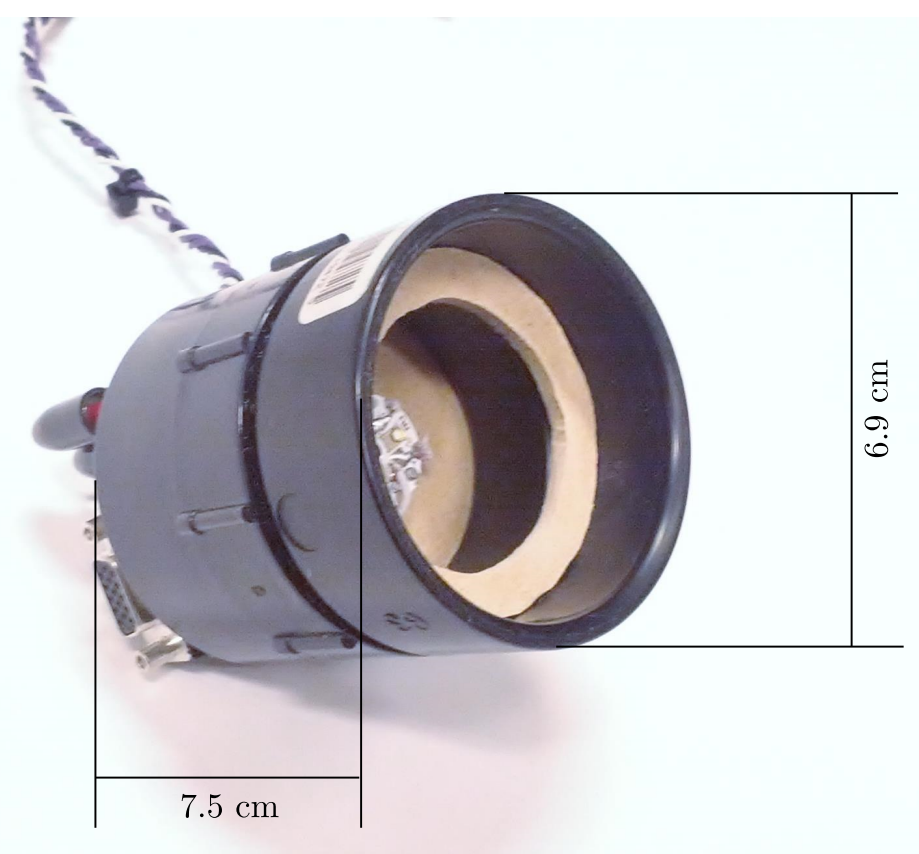

Figure 4.3: Photograph of transmitter

\section{Analog Driver}

The LED driver circuit, shown in Figures 4.4 and 4.5, is an operational amplifier and bipolar junction transistor configured as a linear variable current sink. The circuit in Figure 4.4a demonstrates the basic principal behind the current driver, while the circuit in Figure 4.4b represents the complete current driver that contains an attenuator for signal conditioning, as well as additional feedback components to improve circuit stability. Three channels of the LT1365 operational amplifier [89] were used to construct three current sinks, where $R_{L}$ acts as a current sensor for the amount of current being sunk into the LED via the 2SD2358 NPN BJT [90]. The voltage across $R_{L}$ feeds back into the negative input of the operational amplifier, such that if a potential $(v)$ is applied to the non inverting input of the device, the operational amplifier will increase or decrease its output voltage until the current through $R_{L}$ 


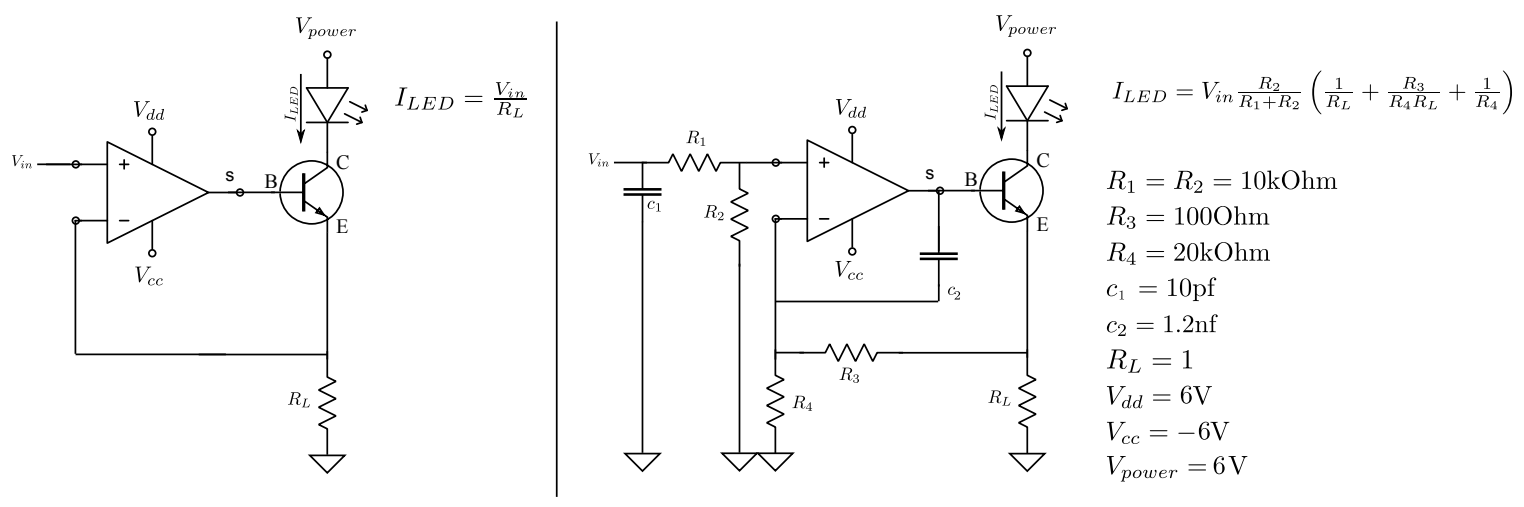

(a)

(b)

Figure 4.4: Linear variable current driver

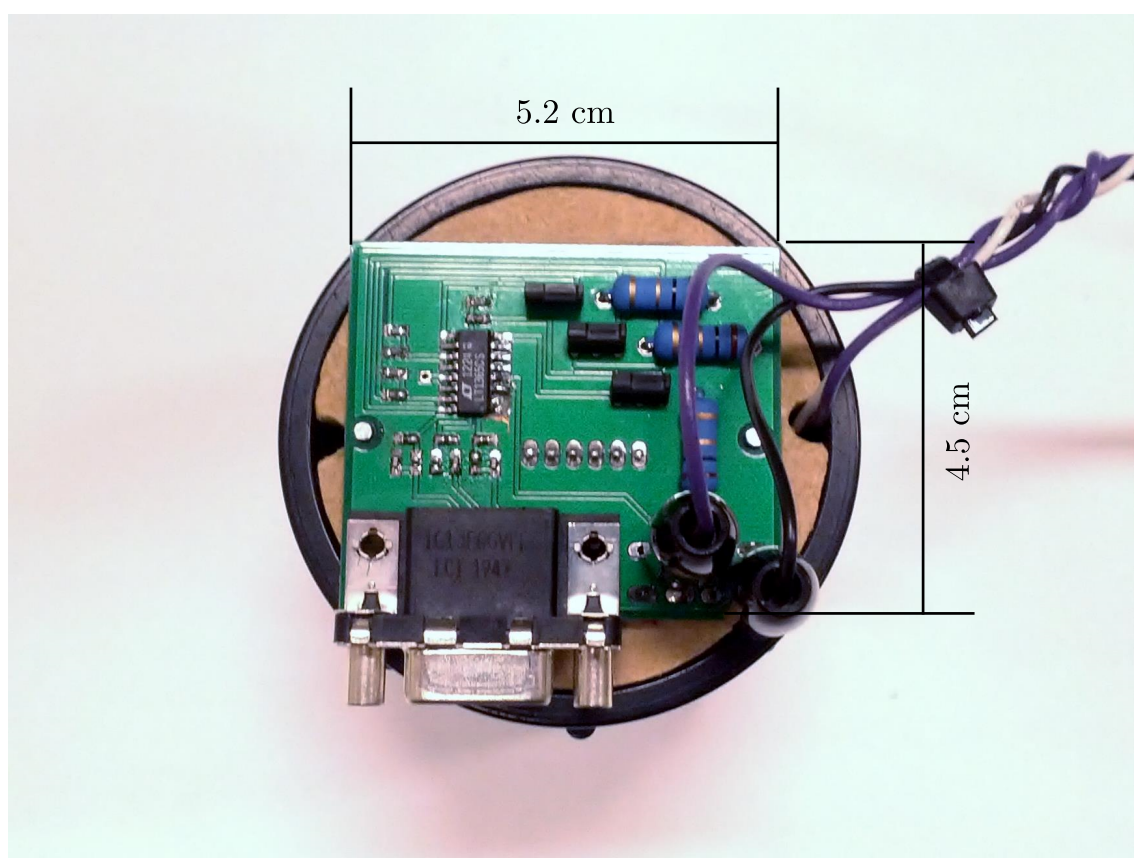

Figure 4.5: Photograph of 3-channel linear current driver 
is equal to $v / R_{L}$. Provided $R_{4} \gg R_{3}$, the drive currents through the LEDs of the complete driver are nearly identical to the current of the simplified driver, scaled down by the attenuation ratio set to $R_{1}$ and $R_{2}$. The capacitor at the input of the complete driver is intended to reduce the overshoot of the DAC output as it may cause the current driver to become unstable. The open collector nature of the driver allows the driver to easily sink 0 to $1 \mathrm{amp}$ with the high voltage headroom, typically 3 volts, required by a single high brightness LED [91]

\section{Light Emitting Diodes}

The luminary, shown in Figure 4.6, used in this experiment was provided by Luxeon Star LEDs, a wholesaler of the Philips Rebel brand of LEDs [92]. The luminary consists of a red, green, and blue LED [91], each on its own substrate, mounted on an aluminum core printed circuit board. Figure 4.7 depicts the clearly non-linear relationship between the measured output radiant flux versus drive current of each LED. The flux was measured from 0 to 0.7 Amps for each LED, and all measurements for a given LED were normalized by the radiant flux corresponding to the flux at 0.7 Amps of the respective colored LED. Measurement of radiant flux versus drive current was achieved by applying a variable direct current independently to each LED, in series with a one ohm resistor, using the ABRA AB-330CUL variable current power

supply with built in ammeter [93], and measuring the LED output intensity using the Thorlabs PM100 optical power meter [94] equipped with the S121B photodetector [95].

Recall that the definition of color mixing presented in (2.11) assumes a linear relationship between the luminous flux of each LED and its driving current. Furthermore, 


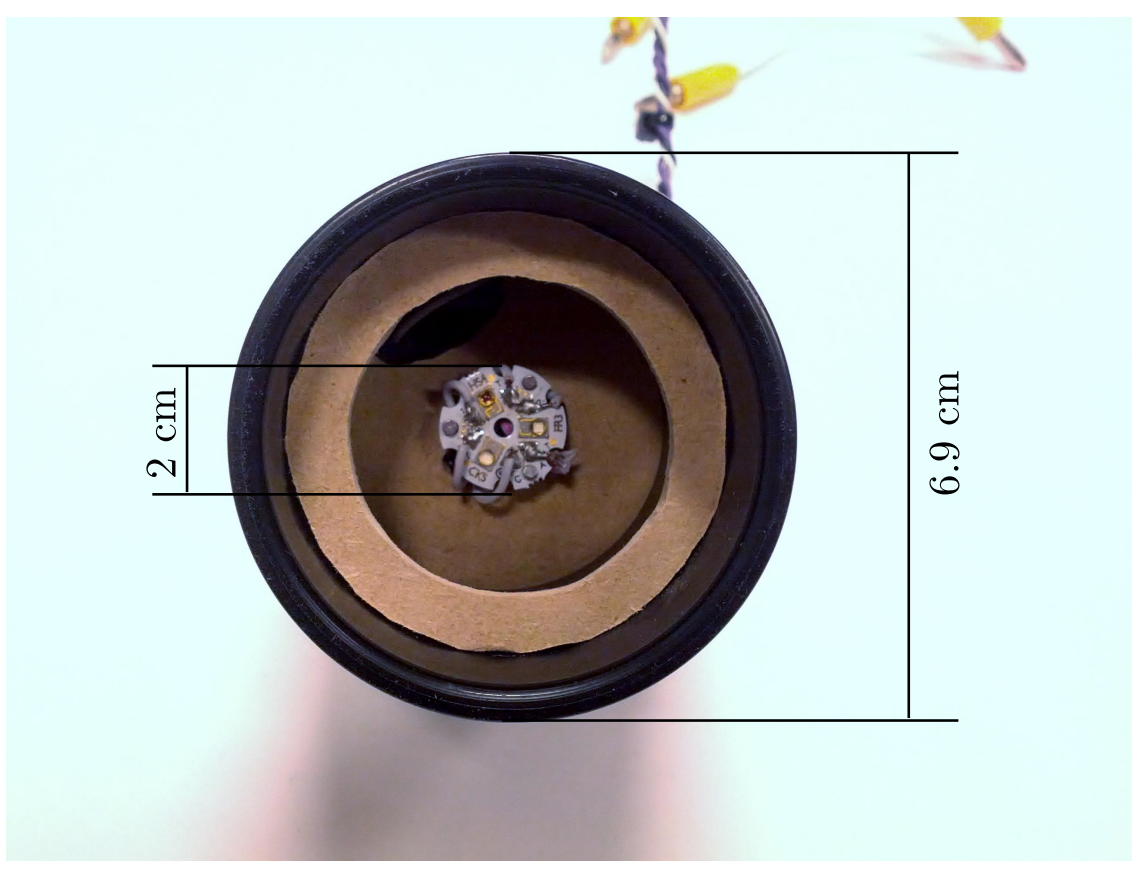

Figure 4.6: Photograph of luminary housed inside transmitter test fixture

the methods discussed in Chapter 3 do not consider non-linear behavior. Therefore, the peak drive current of each LED was limited to $200 \mathrm{~mA}$ in order to reduce the effect of non-linearities on experimental error performance. In this implementation, the first 8 bits of the DAC were assigned to drive the LED up to the $200 \mathrm{~mA}$ current limit while the remaining 2 bits were reserved to allow the driver to exceed the 200 $\mathrm{mA}$ current limit for future applications, such as linearizing the LED output power.

\subsubsection{Receiver Elements}

Figure 4.8 is a functional block diagram of the receiver. The receiver consisted of a single amplified photodiode, a one inch focal length bi-convex imaging optical concentrator, a set of colored polyester optical filters, a digital sampling oscilloscope (DSO) and MATLAB scripts for post measurement digital signal procession (DSP). 


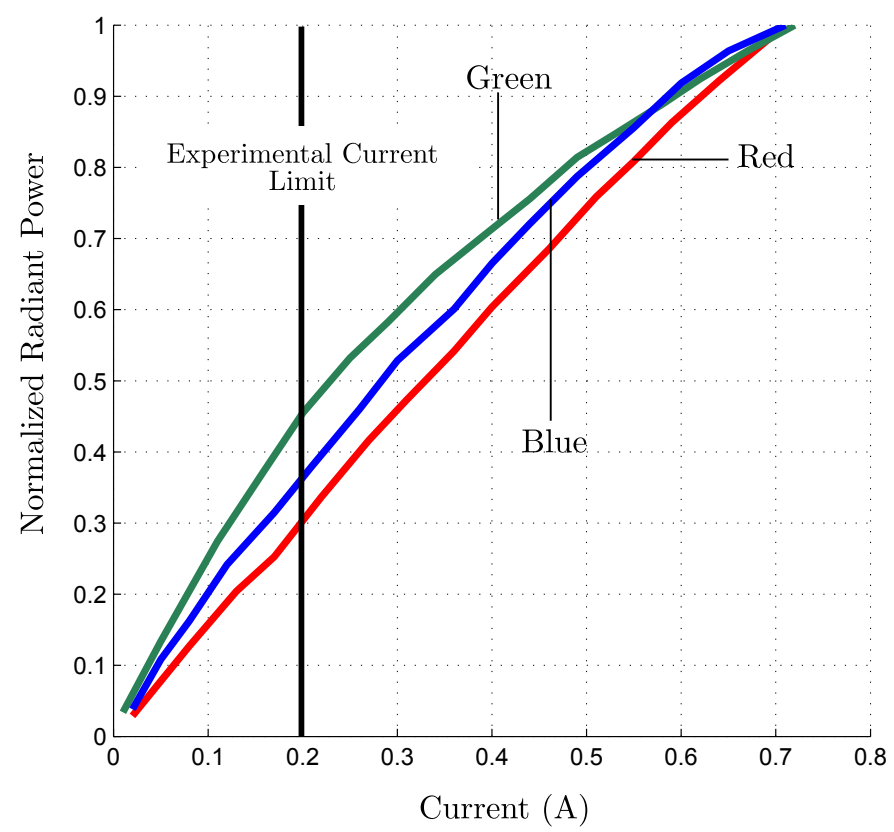

Figure 4.7: DC linearity of each LED used in the experiment

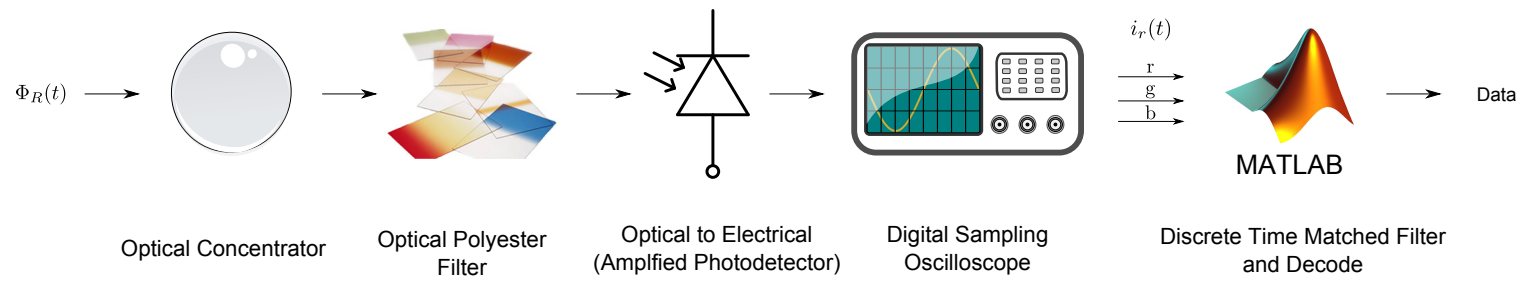

Figure 4.8: Functional block diagram of receiver 


\section{Front End Detector}

The optical front end consisted of an amplified photodetector, optical concentrator, and colored filters. Figure 4.9 shows the unfiltered responsivity of the PDA36A amplified photodetector [96] used at the front end of the receiver. The PDA36A was configured to operate with a $0 \mathrm{~dB}$ gain and a $10 \mathrm{MHz}$ bandwidth, with the responsivity of the device adjusted through the use of colored polyester optical filters. Polyester filters were chosen because they are thin, low cost, highly transmissive in the passband, and tend to have spectral widths similar to that of LEDs. The red [97], green [98], and blue [99] optical filters that were used in this experiment were provided by LEE Filters. The responsivity of the PDA 36A, when filtered, is found by weighting its responsivity with the transmission spectra of the current filter; the optical transfer functions of each filter are shown in Figure 4.10. Finally, a glass biconvex lens, with a one inch diameter and one inch focal length, was used to concentrate light onto the filtered detector surface.

\section{Data Acquisition and Demodulation}

The Agilent Infiniium 54855a [100] digital sampling oscilloscope (DSO) was configured to oversample the received symbols, $\mathbf{r}_{\mathbf{i}}$, from (2.40) with an oversampling rate of 25 samples per symbol. Oversampling allowed a matched filter to be implemented in MATLAB. In the case of rectangular pulses, the matched filter is the average of the samples in a given symbol. Recall from (2.26) that the SNR is defined as the mean squared of the signal divided by the variance of the noise. The averaging filter, assuming uncorrelated noise, affects each the received signal of each color channel 


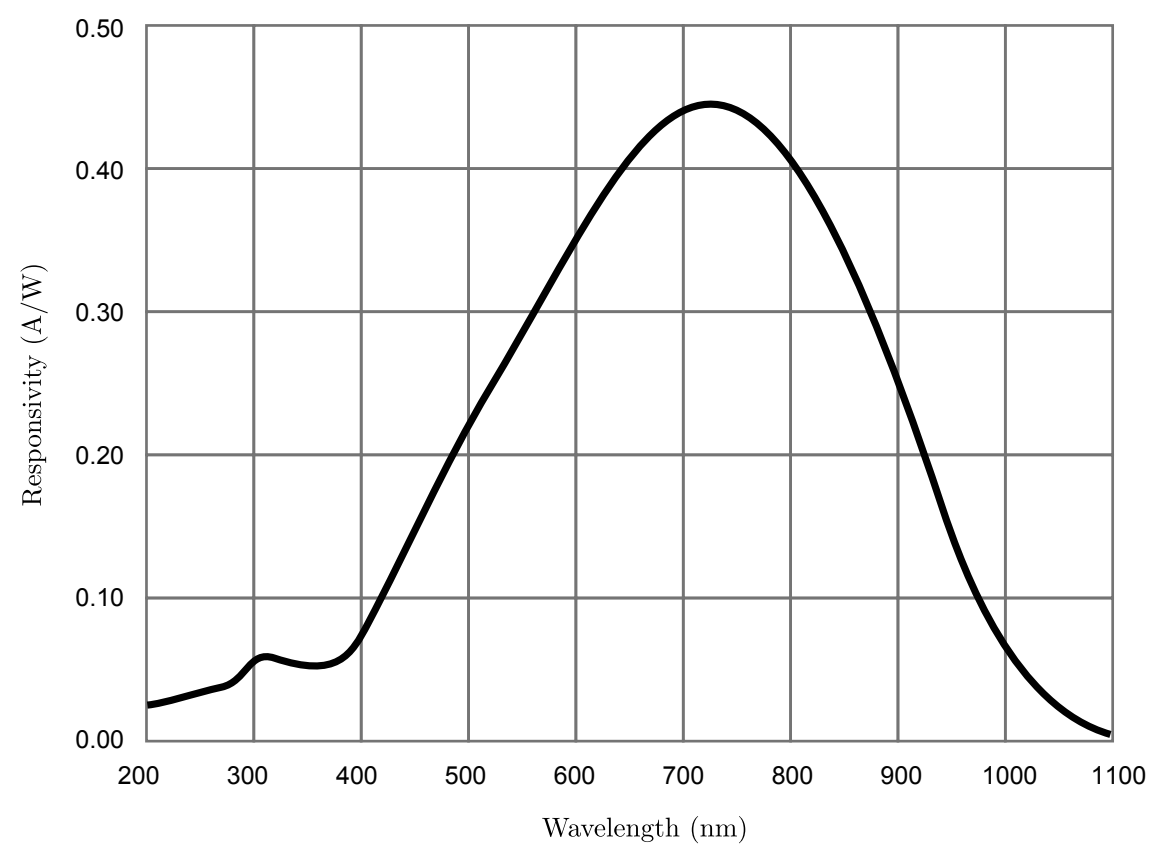

Figure 4.9: Responsivity of the PDA36A variable gain photo detector[96]

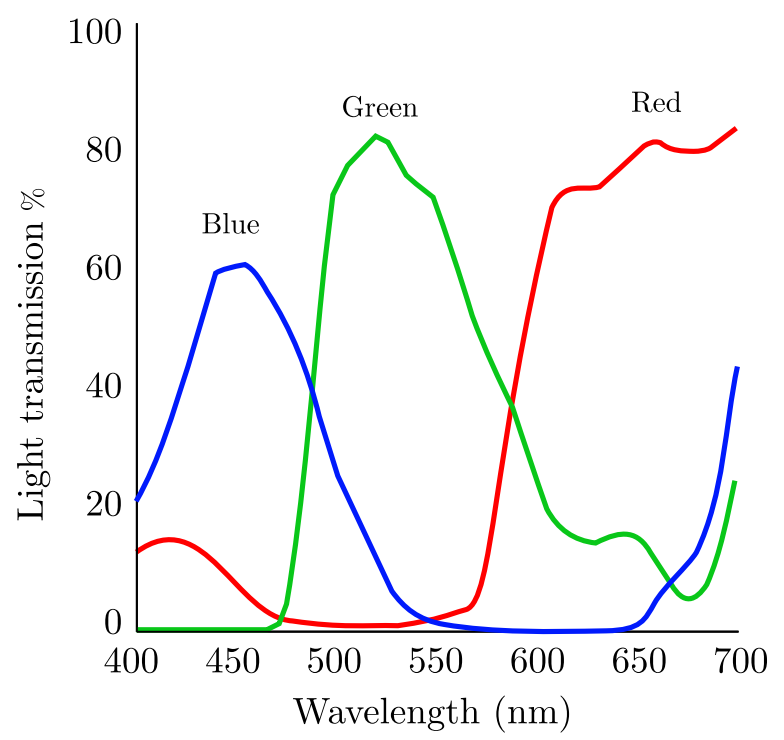

Figure 4.10: Polyester filter transmission spectra [97, 98, 99] 
such that,

$$
\begin{gathered}
\mathbb{E}\left(\frac{1}{N_{s}} \sum_{i}^{N_{s}} \mathbf{r}_{\mathbf{i}, \mathbf{j}}\right)=\mathbf{H s}_{\mathbf{i}} \\
\operatorname{Var}\left(\frac{1}{N_{s}} \sum_{j}^{N_{s}} \mathbf{r}_{\mathbf{i}, \mathbf{j}}\right)=\frac{N_{s}}{N_{s}^{2}} \operatorname{Var}\left(\mathbf{n}_{\mathbf{i}}\right)=\frac{1}{N_{s}} \sigma^{2}
\end{gathered}
$$

where $\mathbf{r}_{\mathbf{i}, \mathbf{j}}$ represents the $j^{\text {th }}$ sample of the $i^{\text {th }}$ symbol, $\sigma^{2}$ is the variance of the unfiltered noise process, $N_{s}$ is the number of samples averaged, Var represents the variance operation, and $\mathbb{E}$ is the expectation. Therefore, the SNR of the received signal can be artificially adjusted by varying the number of samples (up to 25) that are averaged, and allowed for efficient measurement of the SER versus SNR of the experimental channel. Notice that this variable averaging process, in the case of an AWGN channel, is equivalent to either scaling the transmitted optical power through duty cycling, or, assuming LEDs with unlimited bandwidth, varying the data rate. Synchronization was guaranteed as the symbol clock from the transmitter is directly sampled by the receiver. Following the matched filter, a minimum distance decoder was implemented, with knowledge of the measure channel matrix, to decode each symbol.

\subsection{System Characterization}

In order to validate the CSK channel model, shown in (2.40), this section characterizes the system bandwidth, linearity, receiver noise, and channel gain matrix of the CSK compatible VLC link implemented in Section 4.1. Additionally, the photo response of the receiver versus illumination is measured in order to determine the link, in the case of this implementation, between SNR and the useful light power falling on a surface, defined as illuminance in (2.32). 


\subsubsection{Bandwidth and Linearity}

The CSK channel model, (2.40), assumes that the channel is flat and behaves perfectly linearly; however, real devices are band limited and never perfectly linear. Figure 4.11 shows, using the DSO, the measured magnitude, defined as $10 \log _{10}$ of the peak to peak voltage, versus frequency, in hertz, of the driver at the emitter of the BJT, and of the receiver at the output of the photodetector, respectively. It can be seen that the $3 \mathrm{~dB}$ bandwidth of each channel was approximately $3 \mathrm{MHz}$, despite both the LED driver and photodetector having a $10 \mathrm{MHz}$ bandwidth. Thus, the modulation bandwidth of the communication channel was limited by the modulation bandwidth of the LEDs. Therefore, in order for the flat channel assumption to hold,the majority of the power of a CSK modulated signal must lie well within the $3 \mathrm{MHz}$ bandwidth. A single CSK color channel is similar to pulse amplitude modulation, except with non-uniform signal levels that may overlap. By limiting the symbol clock to 100 kilo samples per second $(\mathrm{kS} / \mathrm{s})$ for all experiments, and assuming the spectral density of one channel of a CSK modulated data sequence is similar to that of PAM, $99 \%$ of the modulated signal power, excluding the DC component, is contained within a flat $1 \mathrm{MHz}$ bandwidth [101, p. 233].

Figure 4.12 shows the normalized receiver output, measured independently for each LED using the DSO, against the transmitted DAC level when stepping from 0 to 255 with a $100 \mathrm{kS} / \mathrm{s}$ symbol clock. Using linear regression, it was found that the coefficient of determination for the red, green, and blue channels were 0.9997, 0.9985

and 0.9991 respectively. Furthermore, the spectrum of each channel was analyzed, using the fast Fourier transform feature of the DSO, when modulating each LED with a $100 \mathrm{kHz}, 0$ to $200 \mathrm{~mA}$, sinusoid. Harmonics appeared at integer multiples of 

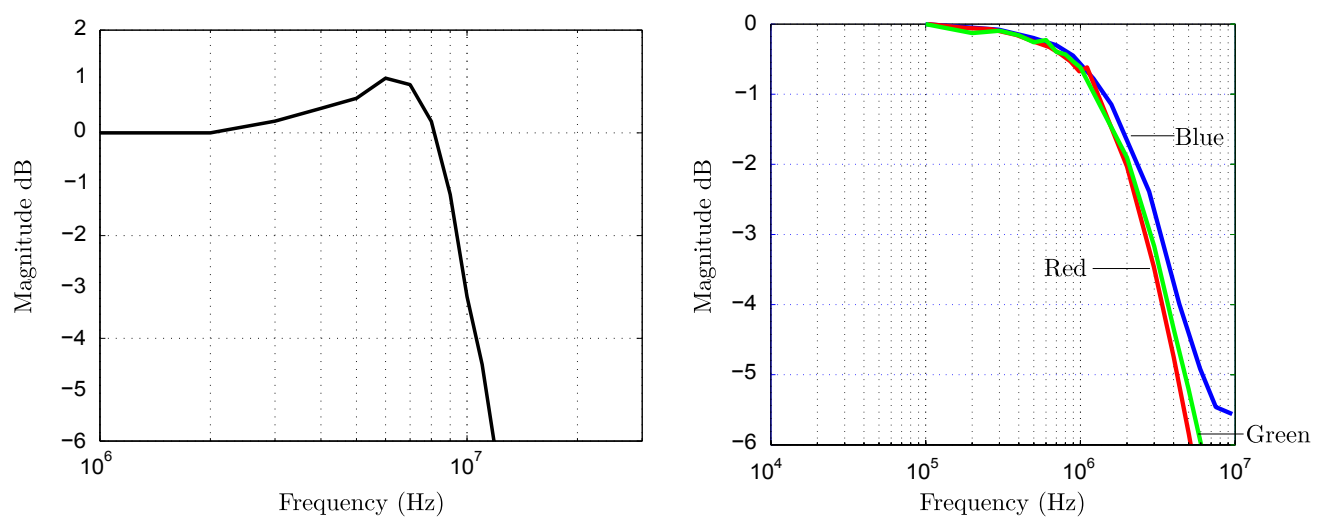

Figure 4.11: Measured magnitude, defined as $10 \log 10\left(v_{\text {peak-peak }}\right)$, versus frequency, in hertz, of a $200 \mathrm{~mA}$ peak to peak drive current, for the CSK channel at the input of the LED string (left) and output amplified photodiode (right). The photodiode bandwidth was measured separability for each colored LED

$100 \mathrm{kHz}$. However, the first harmonic was measured to be approximately $37 \mathrm{~dB}$ down from the primary tone for the blue channel, $35 \mathrm{~dB}$ down from the primary tone for the green channel, and $44 \mathrm{~dB}$ down from the primary tone for the red channel. Therefore, as long as the drive current was limited to $200 \mathrm{~mA}$, the entire system behaved nearly linearly.

\subsubsection{Noise Characterization}

The equivalent baseband discrete time channel model in (2.40) assumes that the received symbol is corrupted by AWGN. The receiver noise is characterized by its covariance matrix and probability distribution function (PDF). The measured PDF, represented by a 1000 bin histogram, and its corresponding quantile-quantile (QQ) plot for 120 mega sample of noise data are shown in Figure 4.13. The QQ plot scatters the quantiles of the histogram of the noise samples against the quantiles of the standard normal; if a QQ plot of a sample vector is linear, the underlying 


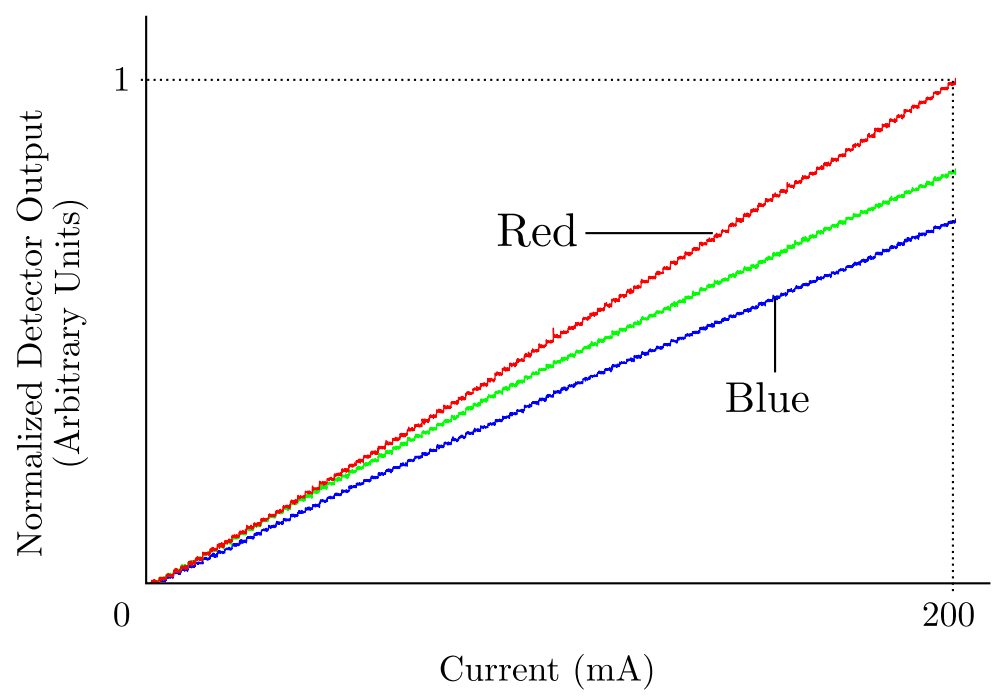

Figure 4.12: Normalized detector output versus drive current

distribution of the noise process is said to be Gaussian. Despite a light skewness in the measured histogram, it can be seen that the PDF of a received noise vector follows a nearly Gaussian distribution, and hence show nearly linear QQ plots.

While the underlying distribution of the channel noise is shown to be Gaussian, the noise samples may not be white in time due to the band-limitedness of the receiver circuitry. To check for whiteness of the noise, the covariance matrix for five time lags of the unprocessed noise was measured,

$$
10^{-6}\left[\begin{array}{ccccc}
0.1415 & 0.0003 & 0.0004 & 0.0006 & 0.0004 \\
0.0003 & 0.1414 & 0.0001 & 0.0005 & 0.0006 \\
0.0004 & 0.0001 & 0.1417 & 0.0002 & 0.0006 \\
0.0006 & 0.0005 & 0.0002 & 0.1413 & 0.0002 \\
0.0004 & 0.0006 & 0.0006 & 0.0002 & 0.1413
\end{array}\right]
$$

It is clear that the covariance matrix is nearly diagonal, suggesting the received noise 

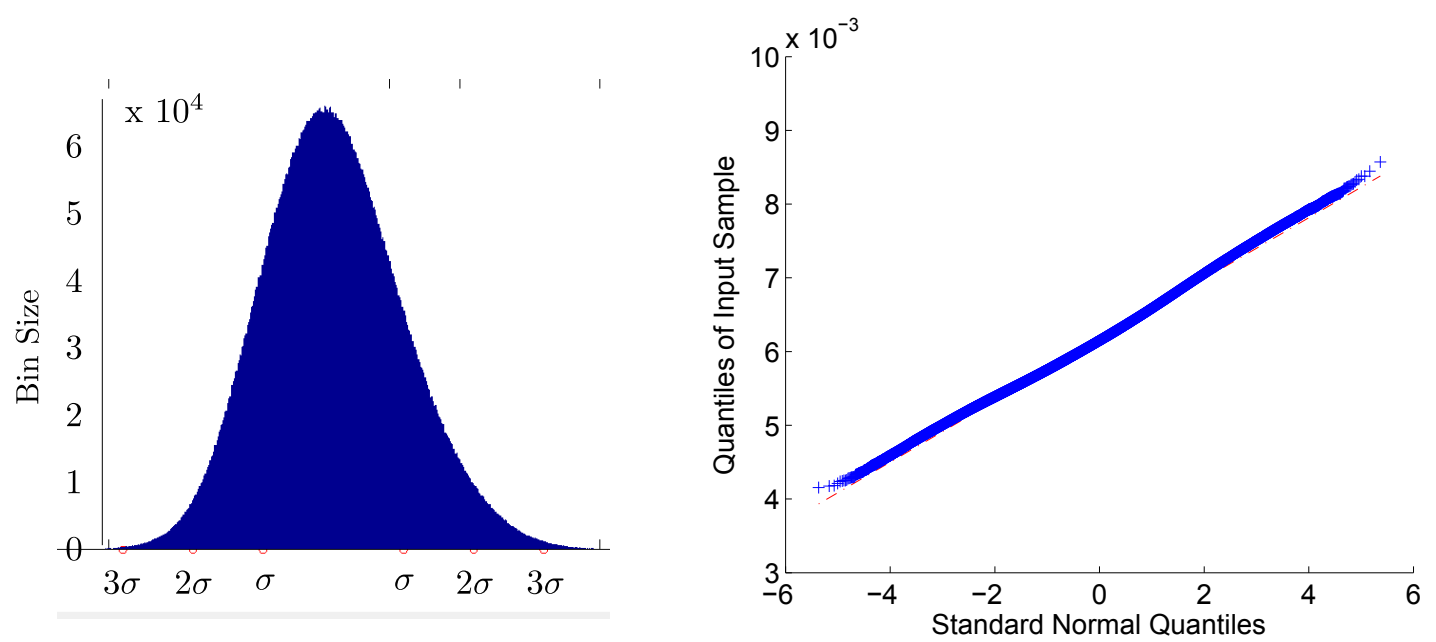

Figure 4.13: Measured PDF and Quantile-Quantile plots of channel noise

vector is uncorrelated in time. This result, along with the measured PDF, confirms that the AWGN assumption for the VLC channel is reasonable. Furthermore, as the measurements for the red, green, and blue channels were performed at different times with the same detector and ambient light levels, the correlation matrix for each of the color bands is identical.

As the ADC of the DSO has an 8 bit resolution over its displayed voltage range, the channel noise measurements included signal dependent quantization noise. It can be shown that when the dynamic range of the input signal is large relative to the quantization step $(\Delta)$, quantization noise behaves similarly to zero mean AWGN, with variance equal to the mean squared error (MSE), approximated as $\Delta^{2} / 12$, of the quantization process [102]. Assuming a worst case scenario that only 7 of the 8 bits, for an $8 \mathrm{mV}$ display range, covered the dynamic range of the signal, the worst case MSE was calculated to be $3.2552083 \mathrm{e}-10$, which is two orders of magnitude less than the total noise variance of the channel. The aforementioned results demonstrate that the available quantization resolution does not significantly impact the channel. 


\begin{tabular}{|c|c|c|c|c|c|c|c|c|}
\hline $\mathrm{R}$ & 1 & 1 & 0 & 0 & 0 & 0 & 0 & 0 \\
\hline $\mathrm{G}$ & 0 & 0 & 1 & 1 & 0 & 0 & 0 & 0 \\
\hline B & 0 & 0 & 0 & 0 & 1 & 1 & 0 & 0 \\
\hline
\end{tabular}

Figure 4.14: Orthogonal "Walsh" code

\subsubsection{Channel Estimation}

As the current drawn by each LED is easily verified with the ammeter built into the DC power supply, the constellations discussed in the next chapter were designed based on constant current symbols, discussed in Section 3.1.3, instead of a constant intensity symbols. Therefore, the elements of $\mathbf{s}_{\mathbf{i}}$ in the channel model, (2.40), represent the current levels driven into each LED. Furthermore, the elements of the channel matrix, defined in (2.39), represent the ratio of LED drive current to the output voltage of the photodetector.

During channel estimation, the transmitter sends an orthogonal code, seen in Figure 4.14, with a mean current of $100 \mathrm{~mA}$ and a symbol clock of $100 \mathrm{kS} / \mathrm{s}$. The DSO measures the mean of each receive color band during each symbol period of the orthogonal code. For example, during the first symbol of the code, only the red LED is active; during this time the receiver is able to measure the contribution of the red LED to the red, green, and blue receive bands. To ensure an accurate measurement, the code is sent for a duration of 10 seconds and all samples are averaged. Furthermore, the symbol for which all three light sources are off is used to measure the offset in each detector due to ambient light. The channel matrix of this implementation was 
measured to be,

$$
\mathbf{H}=\left[\begin{array}{ccc}
1 & 0.041935484 & 0.029886148 \\
0.19373814 & 0.664611006 & 0.276565465 \\
0.009487666 & 0.083965844 & 0.420967742
\end{array}\right]
$$

The above $\mathbf{H}$ has had each of its elements scaled such that the first element of the matrix is unity. Most importantly, $\mathbf{H}$ is clearly full rank, guaranteeing a unique mapping between the transmitter a receiver signal spaces.

In addition to the emission spectra of the LEDs and transmission spectrum of the filters, the channel matrix is dependent on distance and orientation of the receiver to the transmitter. However, in this experiment the receiver and transmitter are always directly aligned along the optical axis of the receiver. Therefore, the channel matrix at any test point is a scaled version of $\mathbf{H}$, and it is this matrix that was used in the design of all constellations in the next chapter.

\subsubsection{Illuminance and Signal to Noise Ratio}

Section 2.4 discussed that the SNR of the channel is directly related to the total illuminance available at the receiver surface. Furthermore, the illuminance provided by a luminary is standardized, suggesting that the notion of SER versus illuminance is more important than SER versus SNR in VLC. Since CSK has three independent channels, the SNR of a CSK signal is defined as the sum of the SNRs of the three color channels. As the induced photocurrent per unit illuminance depends on the wavelength of light illuminating the detector, achieving the relationship between the SNR and illuminance requires that the contribution of each colored LED to the total 


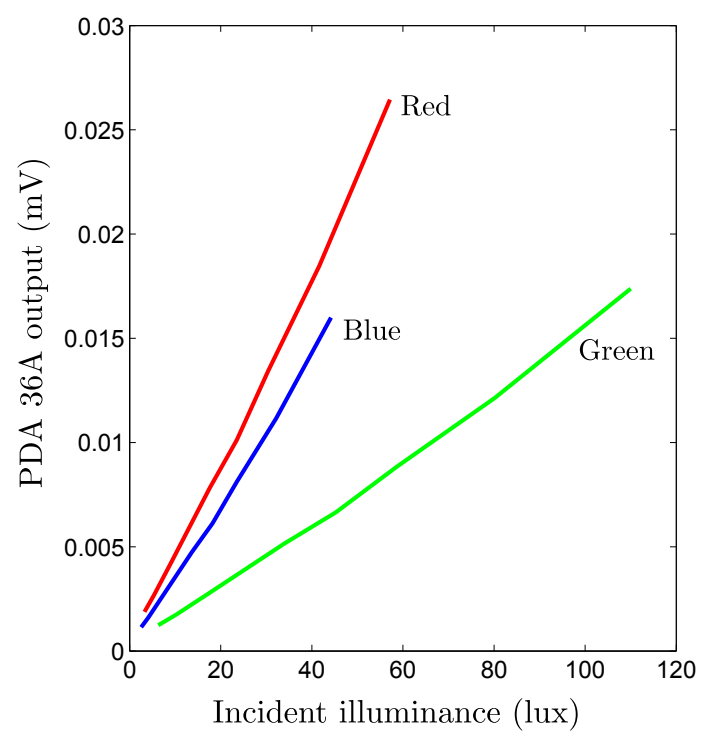

Figure 4.15: Measured photodiode output versus illuminance

received SNR be known, as well as the photo response versus illuminance for each LED.

Figure 4.15 shows the photodetector output measured with the DSO, in millivolts, for the PDA 36A photodetector against the illuminance, measured with the Extech HD400 Heavy Duty Light Meter luxometer, of each LED [103]. The photodetector output versus illuminance was measured by independently driving each LED with an average current of $150 \mathrm{~mA}$, with the illuminance at the detector surface adjusted by varying the physical distance between the transmitter and receiver. The strongest photo response was in the red band due to the strong red responsivity of the photodiode and the use of a highly transmissive long pass red filter. Due to the poor efficiency of green LEDs, and the fact that green light is weighted heaviest by the CIE luminosity function, the green response was the weakest, despite the poorer blue response of the photodiode to blue wavelengths over green wavelengths. 
For a given SNR, the contribution of an LED to total illumination depends on both the measured channel gain matrix, $\mathbf{H}$, as well as the mean current of each LED, $\mathbf{d}_{\mathbf{c}}$, in a given constellation. The sum of the elements of a single column of $\mathbf{H}$ represents the relative combined photo response, across all three received bands, of the detector to each LED when driven with the same mean current. Therefore, the relative contribution of each LED to the total mean photocurrent, denoted by $\mathbf{w}$, is expressed by,

$$
\begin{aligned}
\mathbf{w} & =M \frac{\mathbf{k}}{\|\mathbf{k}\|_{1}} \\
\mathbf{k} & =\mathbf{d}_{\mathbf{c}} \cdot\left[\begin{array}{lll}
1 & 1 & 1
\end{array}\right] \mathbf{H} \\
M & =\sqrt{S N R} \sigma
\end{aligned}
$$

where represents the inner product, and $M$ is the total photo response of the red, green, and blue receive channels for a given SNR and noise variance. With the weight of the contribution of each colored LED to the received mean known the illuminance from each LED that must have been incident on the detector to produce the total received SNR can be calculated by simply looking up the illuminance corresponding to the photo response in Figure 4.15. The total illuminance incident on the detector is the sum of the illuminance of the red, green, and blue LEDs.

\subsection{Conclusions}

In this chapter, the implementation and characterization of a CSK enabled VLC channel was discussed. It was shown that the transmitter driver maintained strong linearity over a $200 \mathrm{~mA}$ dynamic range, but degraded for higher currents due to the non linearity of the LEDs themselves. Additionally, the bandwidth of the system was 
shown to be limited to approximately $3 \mathrm{MHz}$ by the LEDs, as the analog driver itself maintained a bandwidth of $10 \mathrm{MHz}$.

The channel gain matrix was shown to be non diagonal with significant overlap between channels, yet still non singular, allowing it to be inverted. Finally, the noise at the receiver was shown to have a Gaussian PDF with a diagonal correlation matrix, supporting the AWGN assumptions made in the previous chapter. In the next chapter, various constellations are designed based on the measured channel matrix and noise properties. Furthermore, the SER of each constellation is simulated and experimentally measured against both electrical SNR and illuminance. 


\section{Chapter 5}

\section{Simulated and Experimental Error}

\section{Performance}

In the previous chapter the experimental implementation and characterization of a CSK communication channel were discussed. This chapter, discusses how constellations were designed using the methods outlined in Section 2.6 and Section 3.4 based on the channel matrix measured in Section 4.2.3. The SER of each constellation is then simulated and measured over different illumination levels. Comparisons between the proposed method and the IEEE design rules a drawn, and alphabet selection guidelines are provided based on required illumination. 


\subsection{Constellation Design for the Implemented CSK Channel}

This section discusses how the design methods, proposed in Section 3.4, for two dimensional constellations were used to design CSK constellations. Furthermore, constellations were designed for the eight Energy Star CCTs, listed in Section 2.2, in order to draw comparisons between operating color and communications performance. The study was limited to constellations of 4, 8 and 16 points as larger constellations have not been standardized by the IEEE; therefore, no benchmark for comparison exists.

\subsubsection{Constraint Formulation}

Figure 5.1 depicts both the set (plane) of all transmittable symbols, and the corresponding normalized design space, defined by (3.4), including the color balance locations for the Energy Star standard CCTs. As mentioned in Section 4.2.3, constellations are designed based on the constant current criteria. No peak constraints are imposed on the current of each independent color channel leading the set of all possible transmit symbols to lie on a triangular plane of constant current as seen in Figure 5.1. As the shape of the constraint region is scale invariant, the current was assumed to be limited to 1 Amp for optimization, and then scaled down to $200 \mathrm{~mA}$ 

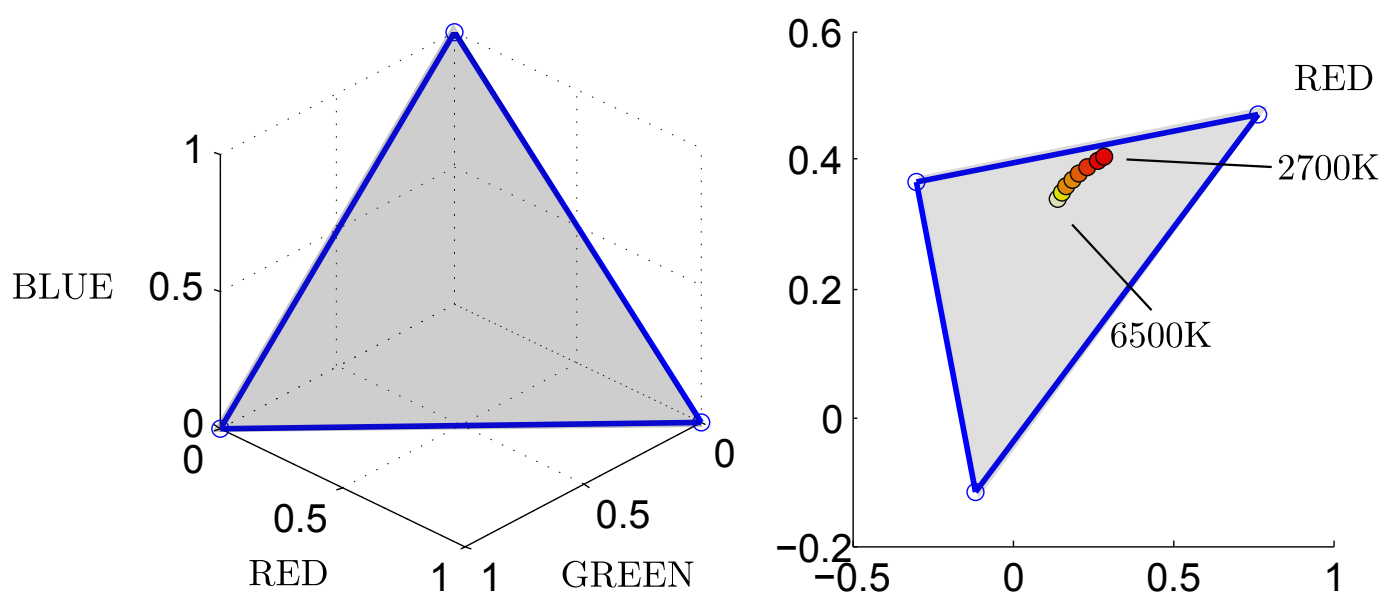

Figure 5.1: All possible transmit symbol (left), normalized design space with color temperature marked (right)

after optimization. The channel matrix used for optimization is as in (4.4),

$$
\mathbf{H}=\left[\begin{array}{ccc}
1 & 0.041935484 & 0.029886148 \\
0.19373814 & 0.664611006 & 0.276565465 \\
0.009487666 & 0.083965844 & 0.420967742
\end{array}\right]
$$

In this case, the vertices of the region bounding all possible transmit symbols, $\mathbf{s}_{\mathbf{i}}$, are represented by the points formed by the columns of the identity matrix. Therefore, the vertices of the region bounding all possible receive symbols are represented by the points formed by the columns of $\mathbf{H}$. The orthographic projection of $\mathbf{H}$,

$$
\mathbf{P}_{\mathbf{r}} \mathbf{H}=\left[\begin{array}{ccc}
0.7665 & -0.2983 & -0.1134 \\
0.4707 & 0.3647 & -0.1150 \\
0.4780 & 0.4780 & 0.4780
\end{array}\right]
$$


Table 5.1: Philips Luxeon Rebel Data-sheet Specifications [91]

\begin{tabular}{|c|c|c|}
\hline \hline Color & Lumens @ $350 \mathrm{~mA}$ & Chromaticity \\
\hline RED & 53 & $(0.703708691,0.296217118)$ \\
\hline GREEN & 102 & $(0.154722061,0.805863545)$ \\
\hline BLUE & 41 & $(0.124118477,0.057802513)$ \\
\hline
\end{tabular}

where $\mathbf{P}_{\mathbf{r}}$ is the orthographic projection matrix defined in (3.4), is required to define the bounding vertices of the normalized design space. Notice that the elements of the third row of the projected $\mathbf{H}$ are identical. By truncating the third row, via the truncation operator, $\mathbf{T}$, described in (3.4), the points defined by the columns of the projected $\mathbf{H}$ are the vertices of the normalized design region shown in Figure 5.1.

To define the color mixing relationship of this CSK implementation, the $X Y Z$ tristimulus of each LED were required. The chromaticity and lumen's per ampere, as stated on the data sheet, of each LED are shown in Table 5.1. Applying (2.10) and (2.11) to the data presented in Table 5.1, produces the following color mixing relationship for this CSK implementation,

$$
\left[\begin{array}{ccc}
2.375760 & 0.191996 & 2.147277 \\
1 & 1 & 1 \\
0.000338 & 0.048914 & 14.153012
\end{array}\right] \mathbf{d}=\left[\begin{array}{c}
\frac{X_{d}}{Y_{d}} \\
\frac{Y_{d}}{Y_{d}} \\
\frac{Z_{d}}{Y_{d}}
\end{array}\right]
$$

Furthermore, as constant current symbols are desired, the vector $\mathbf{d}$ must to converted to the vector $\mathbf{d}_{\mathbf{c}}$ using (3.14),

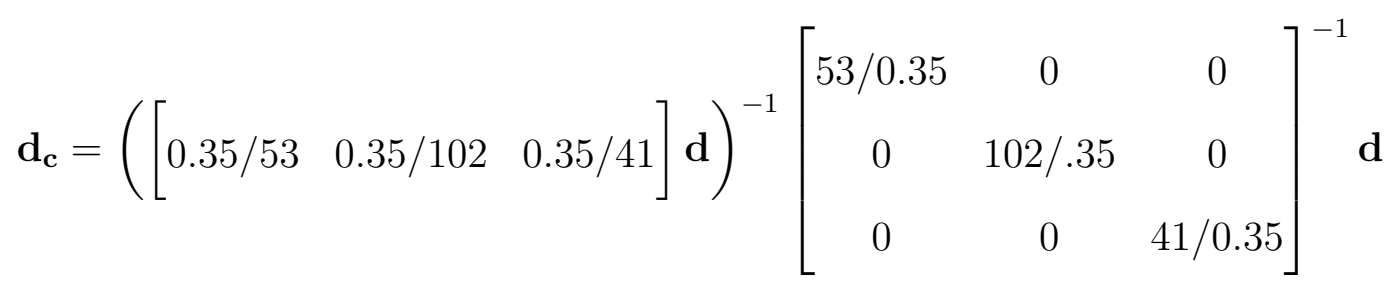


The color points shown in Figure 5.1 (b) represent the locations of all the energy star standard color temperatures in the normalized design space derived by applying (5.4) to the desired CCT, and then projecting each point using (3.4). As more than one chromaticity maps to a given CCT, the exact chromaticities of a black body radiator, heated to the $\mathrm{CCT}$, were used.

\subsubsection{Designed Constellations}

With color mixing, and the normalized design space defined, the design of constellations, optimized with any desired operating chromaticity, was possible through the application of the methods discussed in Sections 2.6 and 3.4. However, the methods discussed in the aforementioned sections assumed that the transmitter is capable of producing a continuous output. The transmitter discussed in Chapter 4 was limited to an 8 bit precession over its full scale output. Therefore, constellations must be quantized before transmission. The quantization rule that was used to convert the optimized constellations into their digital representations was,

$$
\begin{array}{ll}
Q(x) & =\operatorname{sgn}(x) \Delta\left[\frac{|x|}{\Delta}-\frac{1}{2}\right] \\
x & \in[0,1] \\
\Delta & =\frac{1}{2^{8}}
\end{array}
$$

where $Q(x)$ is the quantized symbol and $s g n$ is the signum function. As discussed in Section 4.2.2, quantization introduces noise into the communication system. However, the simulation results presented in this section are those of the quantized constellations. Therefore, when comparing the simulation results to the experimental results 
discussed in the next section, quantization error induced by the quantization of constellations was not a factor. This approach is appropriate as constellations designed with the methods discussed in Chapter 3 were not analytically demonstrated to be globally optimal.

Figures 5.2, 5.3 and 5.4 depict the 4, 8, and 16-CSK two dimensional constellations, where each axis represents drive current, derived from both the IEEE design rules and the proposed CSK optimization method. The minimum distance, $d_{\min }$, before quantization in the normalized design space of each constellation is shown, with the dots representing symbols bounded in the constraint region. The numeric values of each constellation, after quantization, can be found in Appendix A. Each figure also contains the simulated SER versus SNR of each constellation after quantization. The following assumptions were made during simulation:

- The frequency response of the channel is flat

- The system is linear

- The each receive channel is corrupted by AWGN of equal variance

- The channel matrix is known to both transmitter and receiver

- SNR is defined as $P^{2} / \sigma^{2}$ where $P$ is the sum of the means of the red, green, and blue receive channels and $\sigma^{2}$ is the variance of the noise

It can seen that the 4-CSK, 8-CSK and 16-CSK optimized constellations, when no operating color is specified, outperform those designed using the IEEE CSK design 
4-CSK
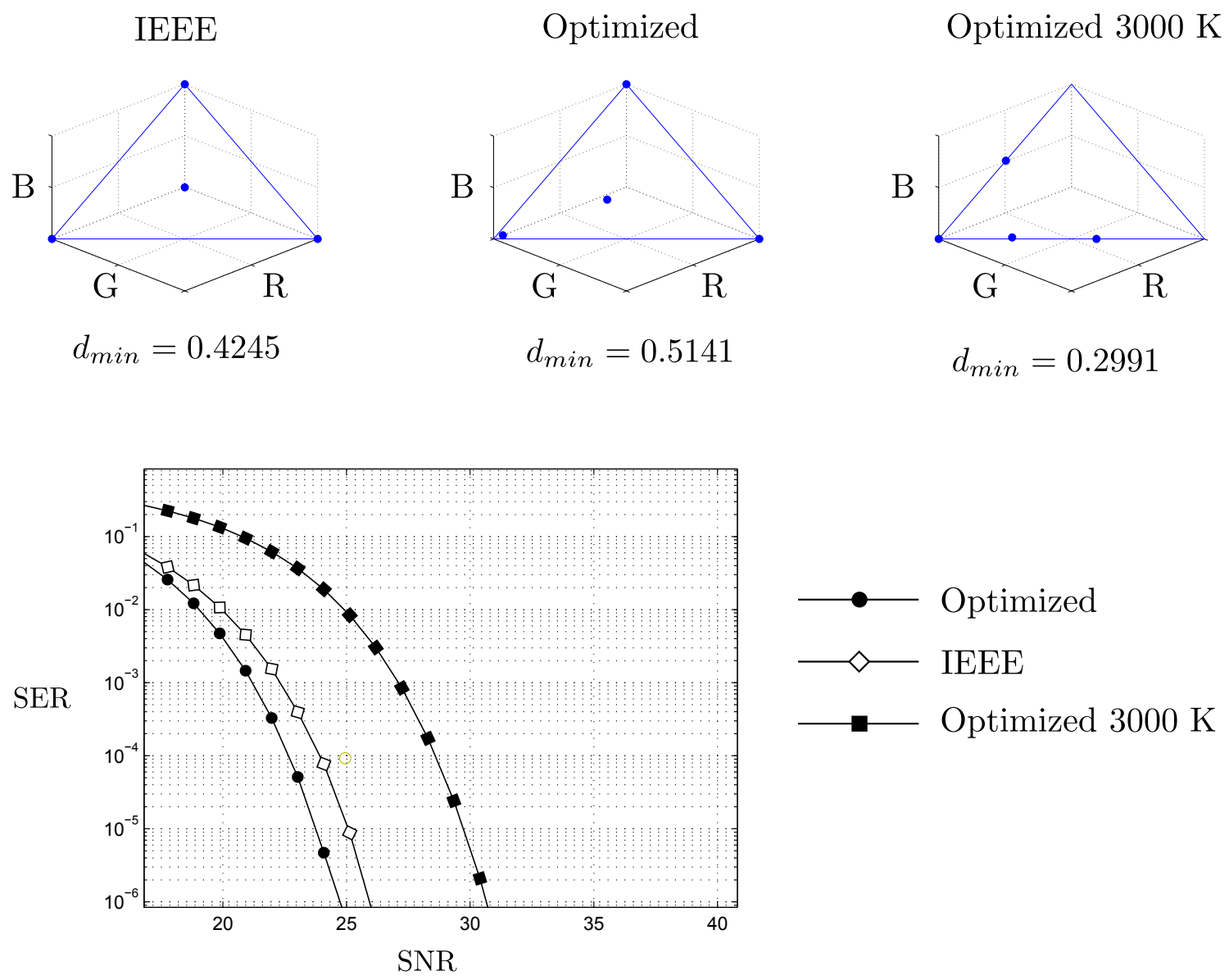

Figure 5.2: Simulated SER versus SNR, minimum distance, and depiction of IEEE 4-CSK, Optimized 4-CSK, and Optimized 3000 K 4-CSK 


\section{8-CSK}

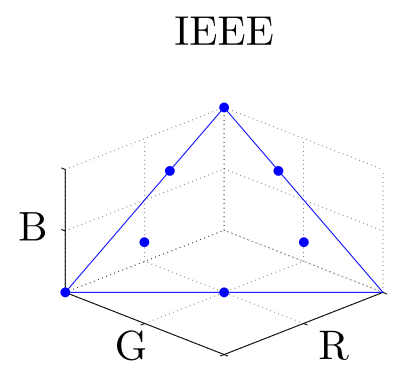

Optimized

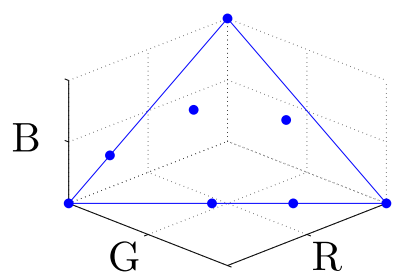

$d_{\text {min }}=0.1758$

$d_{\min }=0.2725$
Optimized $3000 \mathrm{~K}$

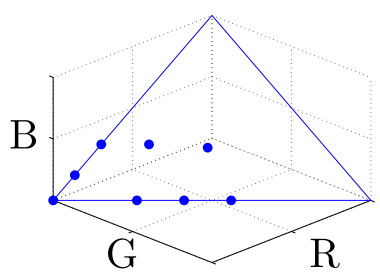

$d_{\min }=0.1445$

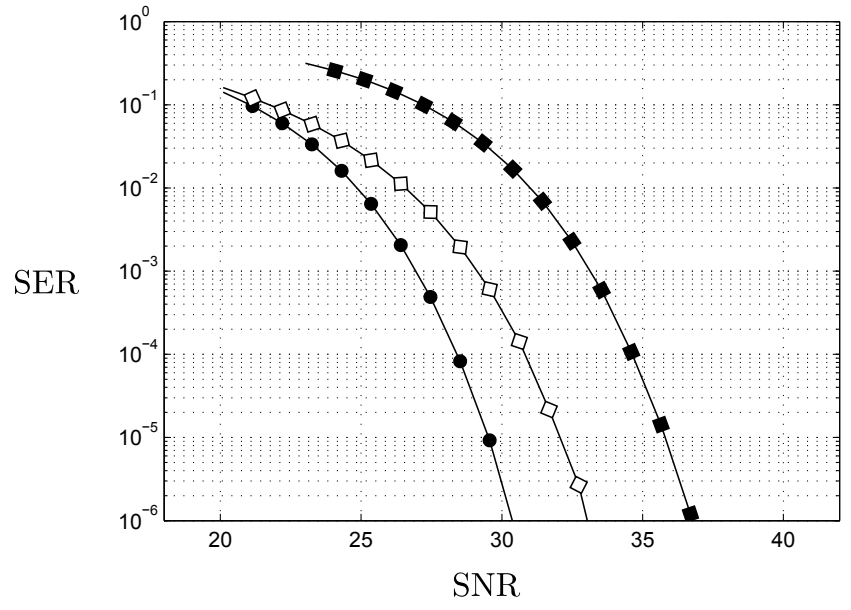

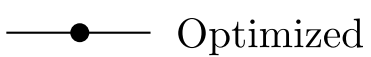

$\diamond$ IEEE

$\longrightarrow$ Optimized $3000 \mathrm{~K}$

Figure 5.3: Simulated SER versus SNR, minimum distance, and depiction of IEEE 8-CSK, Optimized 8-CSK, and Opted 3000 K 8-CSK 


\section{$16-\mathrm{CSK}$}
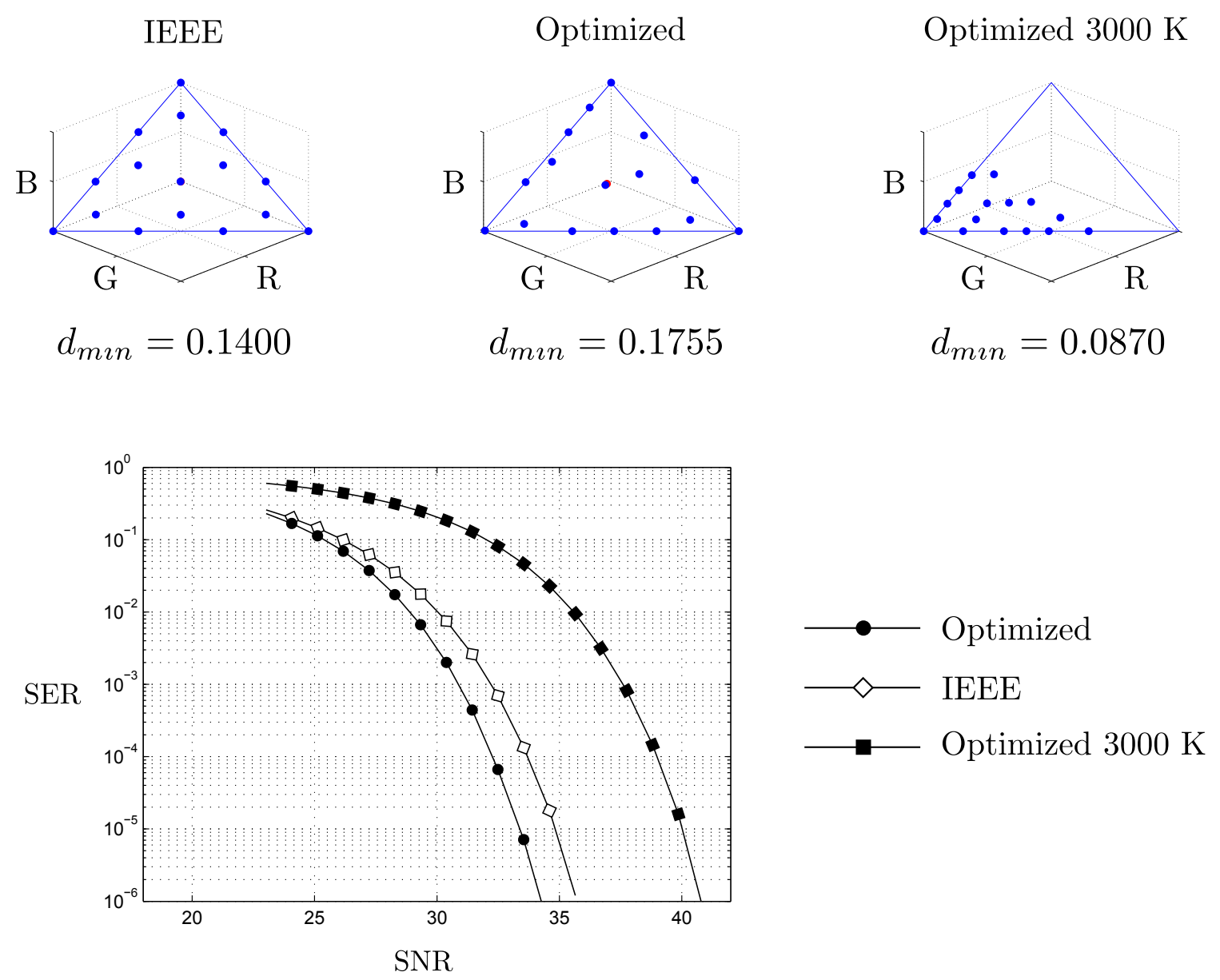

Figure 5.4: Simulated SER versus SNR, minimum distance, and depiction of IEEE 16-CSK, Optimized 16-CSK, and Opted 3000 K 16-CSK 
rules by approximately $1.5 \mathrm{~dB}, 3 \mathrm{~dB}$ and $2 \mathrm{~dB}$ respectively. The aforementioned performance differences agree with the observation that the minimum distances of the optimized constellations show a $21 \%, 55 \%$ and $25 \%$ improvement for 4,8 and 16-CSK respectively, when compared to the IEEE 802.15.7 standard constellations. These observations support the validity of the optimization objective described in (3.18). It is worth noting that the CCTs of the constellations not optimized for a specific chormaticity, including the standard IEEE constellations, are equal to infinity when using the calculation method discussed in Appendix B; this remains true for either constant current or constant luminous flux operation. It is clear that not constraining the chromaticity of a luminary will produce undesirable illumination. Therefore, constellations were also optimized for the typical household lighting CCT of $3000 \mathrm{~K}$. The constellations optimized for $3000 \mathrm{~K}$ incur a performance penalty of 6 $\mathrm{dB}, 6 \mathrm{~dB}, 6.5 \mathrm{~dB}$ respectively when compared to their optimized no specified operating color counter parts.

Clearly constraining the CCT of a luminary had a significant impact on the performance of the CSK enabled VLC link. To examine this difference further, the constellations in Figure 5.5 depict multiple 8-CSK constellations, each optimized for one of the eight standard Energy Star CCTs, where 2700K is near a vertex and 6500K is near the centroid. Furthermore, the SER versus SNR for each constellation in Figure 5.5 are shown in Figure 5.6. It can be seen that the minimum distance of the 8-CSK constellations monotonically increases with color temperature thus providing a gain in SNR. These observations confirm that balancing the constellation away from the centroid of the normalized design space will incur a performance penalty, and immediately suggest that higher color temperatures are favorable for communications. 
While the household may have typical color temperatures of $3000 \mathrm{~K}$, office lighting is typically $6500 \mathrm{~K}$ [63]. Therefore, in the case of office work, the performance penalty for optimizing for $6500 \mathrm{~K}$ versus not specifying an operating color is only $1 \mathrm{~dB}$, with the difference between home and office lighting being approximately $5 \mathrm{~dB}$. Interestingly, the optimized constellation that meets the office lighting color requirement of $6500 \mathrm{~K}$ outperformed the IEEE standard constellation that did not meet Energy Star CCT requirements.

\subsection{Experimental Performance}

This section discusses the measurement of the SER versus SNR for the 4,8 and 16 point constellations shown in Figures 5.2, 5.3, and 5.4 using the experimental communication channel characterized in Chapter 4. Comparisons are drawn between the simulated and experimental results, and potential causes of any discrepancies are discussed.

\subsubsection{Measurement Method}

Before any measurements were taken, all pieces of equipment, including the LEDs and LED driver, were powered up and left idle for 30 minutes to allow them to settle into a nominal operating temperature state. To measure the SER, the modulator selects data randomly through the use of a pseudo random number generator (PRNG). The PRNG has a maximum length of 32768 elements before repeating and is implemented using a 15 bit linear shift feedback register that linearly shifts the register elements from the least significant bit to the most significant bit during each symbol period. 

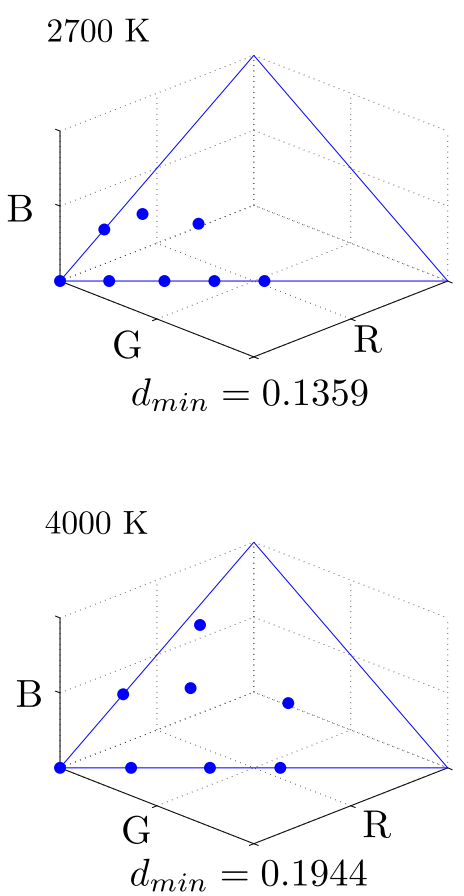

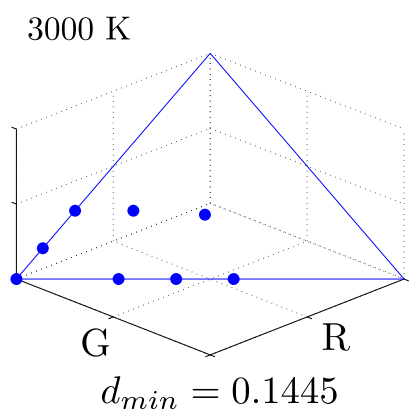

$4500 \mathrm{~K}$

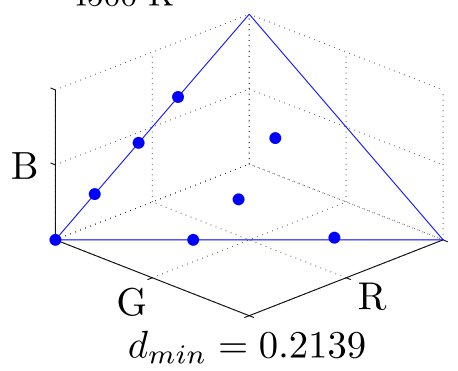

$3500 \mathrm{~K}$

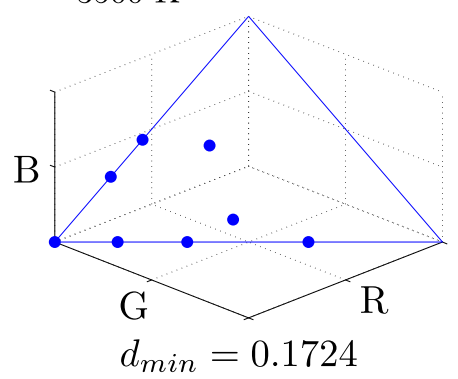

$5000 \mathrm{~K}$

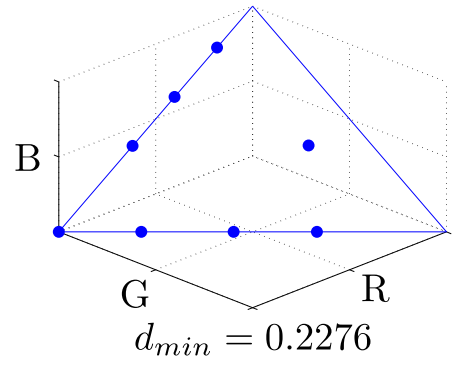

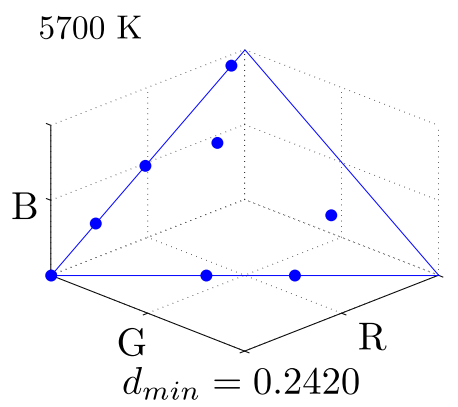

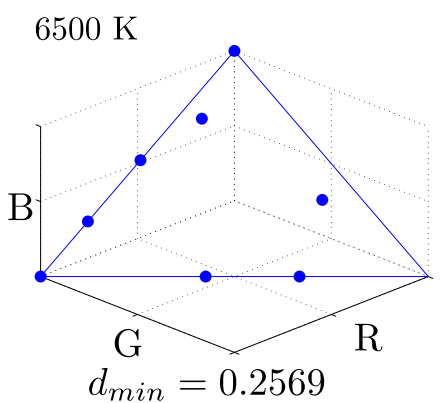

Figure 5.5: 8-CSK optimized for a CCT of 2700K, 3000K, 3500K, 4000K, 4500K, $5000 \mathrm{~K}, 5700 \mathrm{~K}$ and $6500 \mathrm{~K}$ 


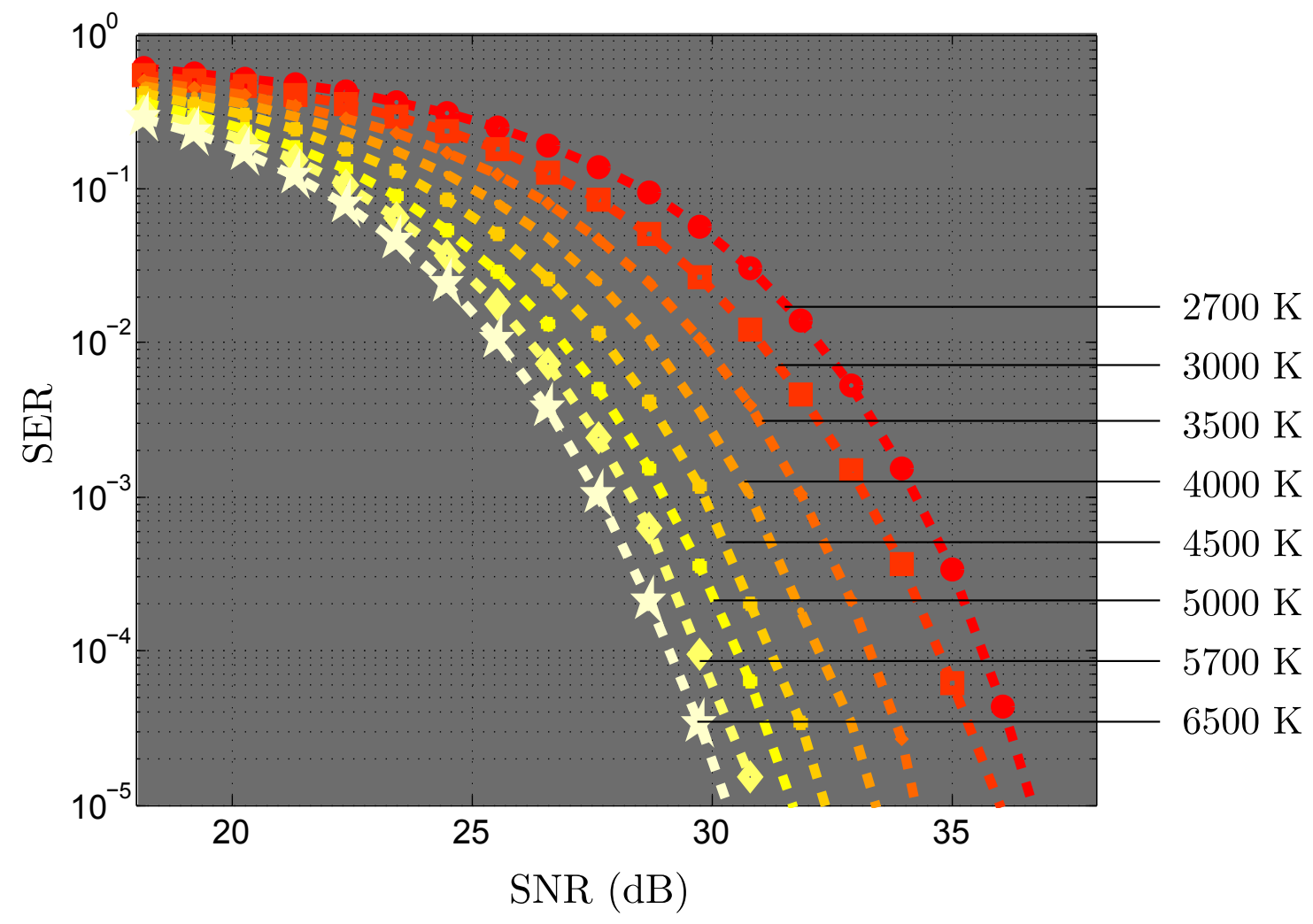

Figure 5.6: Simulated SER vs SNR for the 8-CSK constellations depicted in Figure 5.5 
The exclusive or of bits 13 and 14 are shifted into bit 0 during each shift. The PRNG seed is known to both the transmitter and receiver, where synchronization was achieved through the use of the auxiliary wire discussed in Section 4.1. Additionally, the symbol rate, as discussed in Section 4.2.1, was set to $100 \mathrm{kSa} / \mathrm{s}$. This allowed the channel to act as a flat AWGN channel, as assumed in the optimization objective described in Section 3.2.

For each constellation, a total of 983040 symbols were captured, and the channel matrix was measured using the method described in Section 4.2.3. Data was captured at a single distance with a fixed illumination for each constellation under test. Recall that each color channel must be measured separately and at different times. Therefore, the capture of 983040 symbols corresponds to 30 partitions of repeated data for each of the color channels. Each partition is corrupted by AWGN independent from any other partition. Therefore, to increase the number of data symbols received, each red partition can be paired with any of the green and blue partitions. This allowed for a total of 27000 combinations of partitions, and an effective total symbol count of 884736000 available for the calculation of the SER.

As the data for each constellation was captured at a fixed distance and illumination, the SNR before the matched filter is fixed. The SNR is thus adjusted using the technique described in (4.2), where the SNR of the received signal is varied by changing the number of samples averaged from 1 to 25 . As CSK has three channels, the total signal power is defined as the sum of the means, as recorded by the DSO, of each color channel, minus any offset due to ambient light. The DSP performed by MATLAB then decoded the averaged samples and calculated the SER by generating the expected PRNG sequence using the same seed as the transmitter. 


\subsubsection{Experimental Results}

Figures 5.8, 5.9 and 5.10 report the measured SER versus SNR for the 4, 8 and 16CSK constellations depicted in Figures 5.2, 5.3, and 5.4. The black curves in Figures 5.8, 5.9 and 5.10 are the simulation results from Figures 5.2, 5.3, and 5.4.

Examining the points in each of the experimental figures, it can be seen that experimental SER follows closely to that of the expected simulation results. The discrepancies between simulated and measured results are attributed to various non idealities. One possible explanation for the discrepancies, aside from human error, is a potentially, yet slight, non linear relationship between the transmitted and received symbols. While care was taken to mitigate non linearities in the system by limiting the current of the LEDs, complete suppression was impossible. Therefore, the measured $\mathbf{H}$ cannot perfectly relate the transmitted symbol set to the received symbol set; as a result the receiver does not have perfect channel state information and the decoders decision region centers are slightly off set from their expected location. This effect can be seen in Figure 5.7 which shows an orthographic projection of a received sample of symbols, signified by the blues point clouds, transmitted through the implemented CSK system discussed in Chapter 4. The figure provides examples for the optimal 8-CSK and 3000K 16-CSK constellations where the centers of the decision regions created by the estimated channel matrix are marked with a red cross. It is evident from Figure 5.7 that the centers of the decision regions are not ideally aligned with

the transmitted symbol set, as the ideal regions should be centered at the center of each blue cloud of points. As a result, the maximum likelihood detector will generate additional symbol errors independent of the AWGN potentially leading to an error floor. 


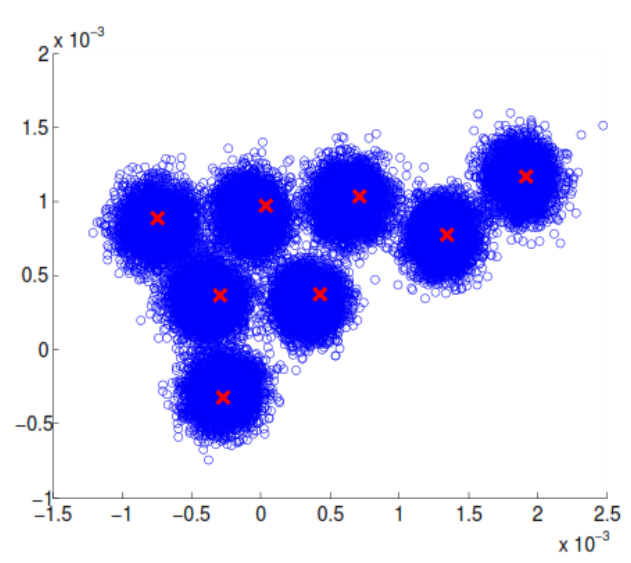

(a)

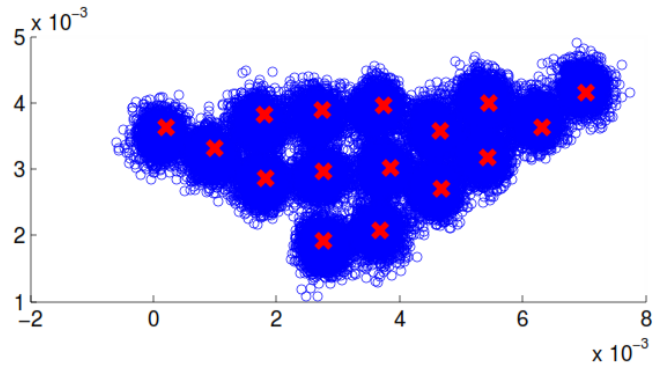

(b)

Figure 5.7: Unitless orthographic projection of received symbols transmitted through the experimental link for a fixed operating current of $200 \mathrm{~mA}$. The decision region centers are marked with a red cross. (a) Optimal 8-CSK (b) 3000K 16-CSK

Evidence of the potential error floor can be seen in Figure 5.10 as the low SNR points of each measured constellation are aligned with the expected simulation results while the high SNR values start to drift to the right of the expected result. The drift is more pronounced in the optimal and IEEE constellations than in the $3000 \mathrm{~K}$ constellations. Examining the $3000 \mathrm{~K}$ constellations in Figures 5.2, 5.3 and 5.4, it can be seen that the dynamic range of the red, green and blue channels of the $3000 \mathrm{~K}$ constellations are approximately half that of the IEEE and optimal constellations; this suggests a higher immunity to non linear effects caused by the LEDs. Furthermore, the IEEE 16-CSK appears to be more prone to non linear effects than even the optimal solution derived using interior point methods. A possible reason for this behavior can be seen in Figure 5.4. Notice that the optimized solutions produce more discrete levels in the red dimensions than the green and blue dimensions compared to the IEEE constellation. As the red channel was shown, in Section 4.2.1, to be the least impacted by non linearities, the optimal solutions were less effected by channel 


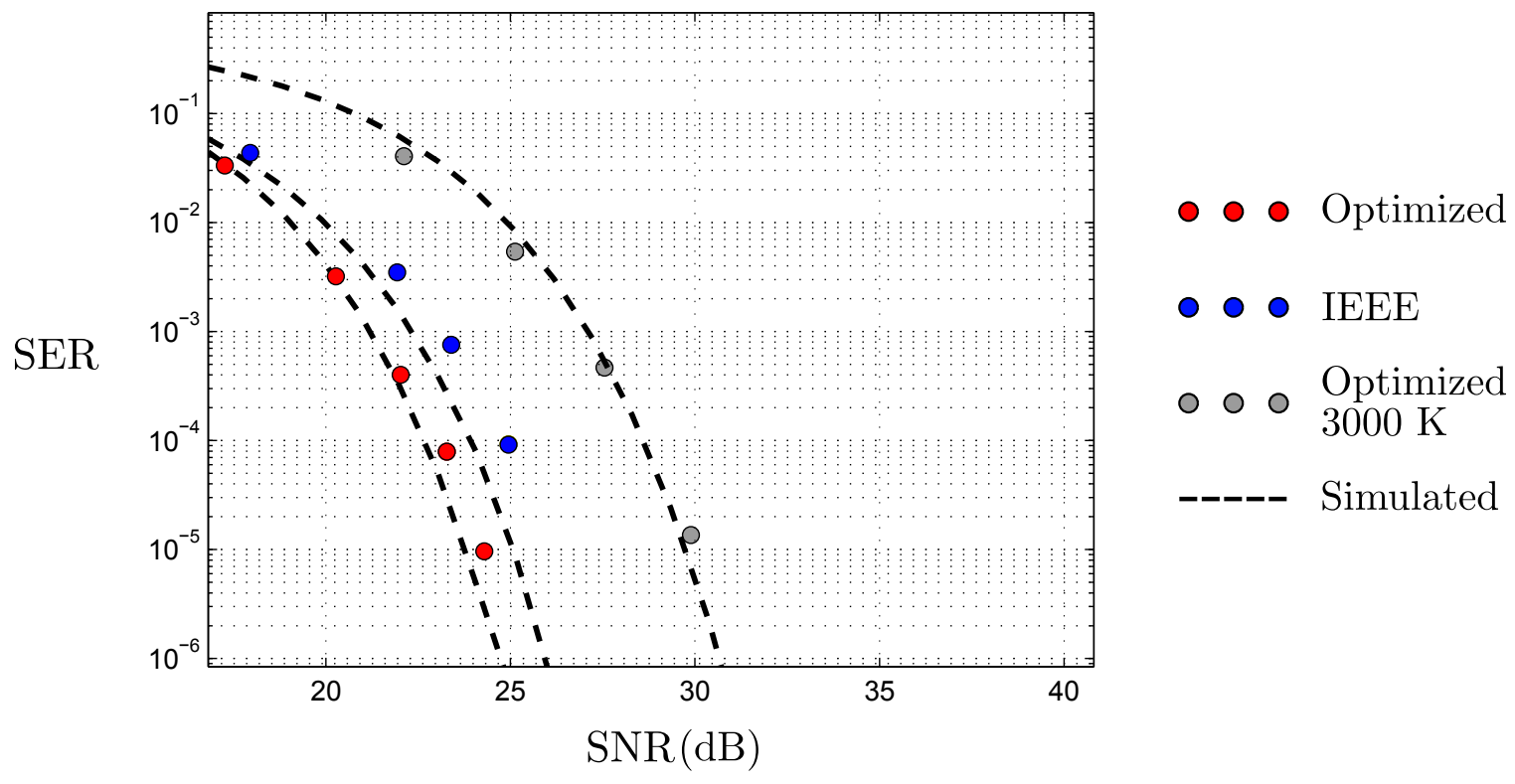

Figure 5.8: Experimental SER versus electrical SNR for 4-CSK

impairments.

While not perfectly representative of the expected simulations results, these results prove that the optimization assumptions made in Chapter 3 were correct as the optimized constellations for 4,8 and 16-CSK outperformed their respective IEEE constellations by approximately $1.5 \mathrm{~dB}, 3 \mathrm{~dB}$ and $3 \mathrm{~dB}$ respectively in a realized CSK channel.

\subsection{SNR versus Illuminance}

While the results presented in Section 5.1.2 and 5.2 are useful from a communications point of view, it is important to remember that the primary concern of VLC is that of illumination. When comparing SER to SNR, the SNR is always the SNR after the matched filter. Therefore, the SNR available for a given illuminance depends on 


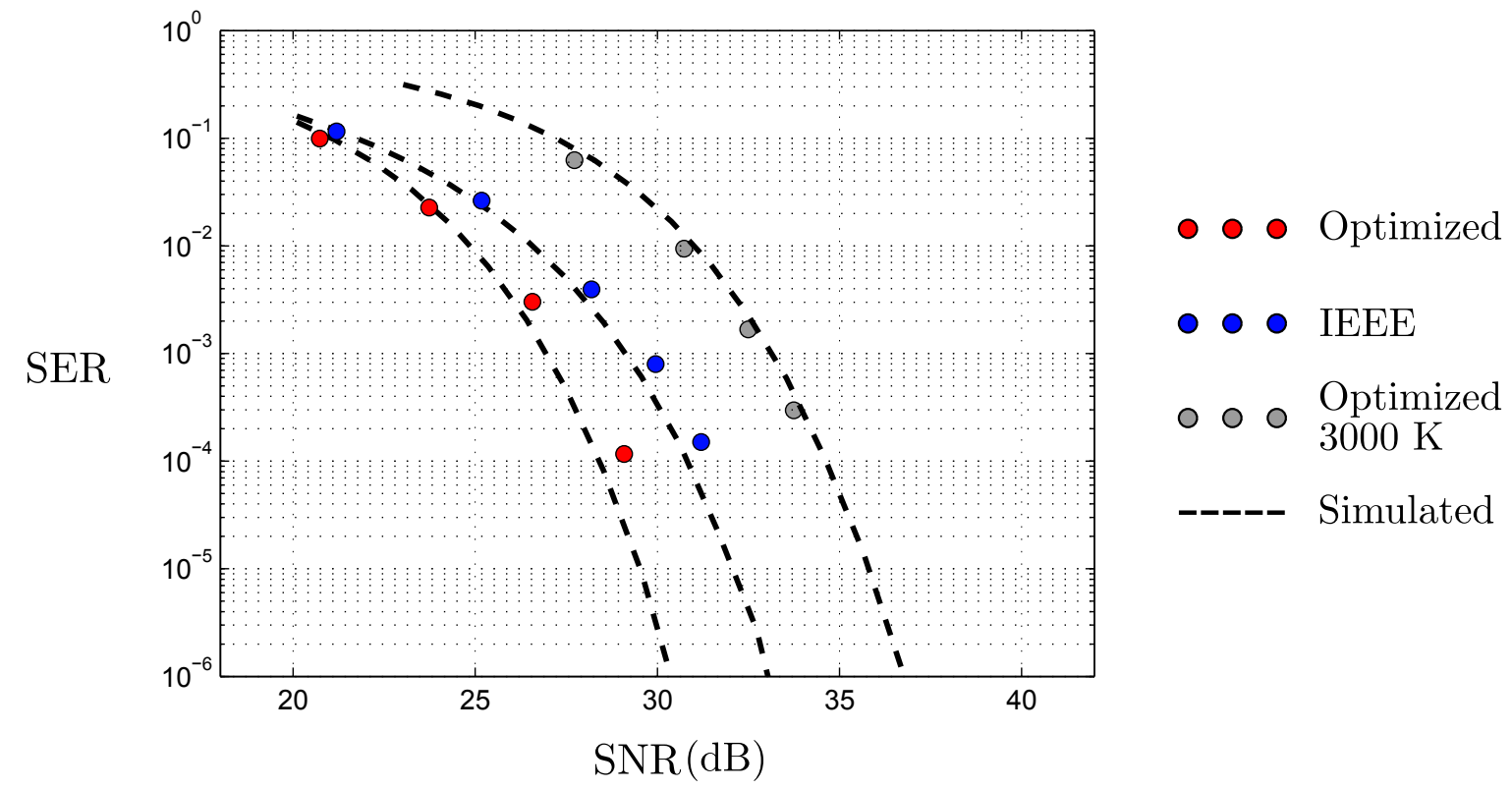

Figure 5.9: Experimental SER versus electrical SNR for 8-CSK

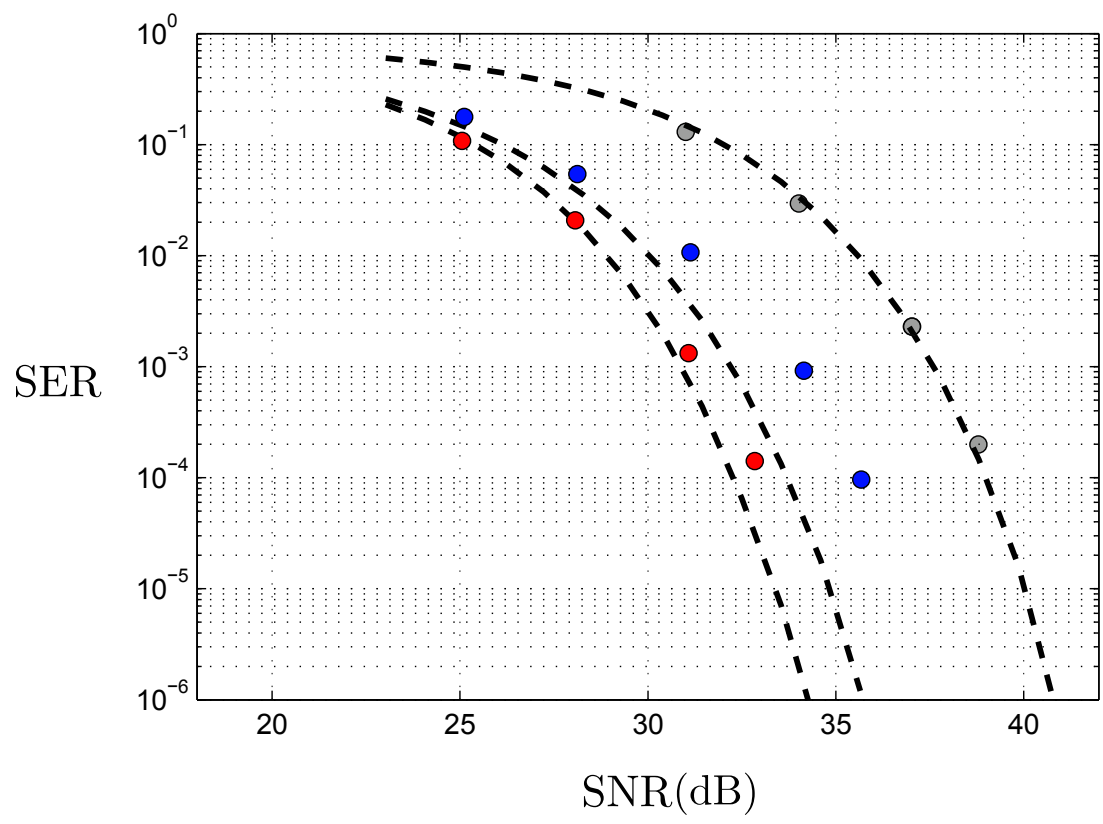

○ ○ Optimized

○ IEEE

○ ○ ○ Optimized

----- Simulated

Figure 5.10: Experimental SER versus electrical SNR for 16-CSK 
the matched filter, as seen in (4.2), which in this experiment was implemented as a discrete time averaging filter. As more samples are averaged by the filter, the smaller the noise variance becomes, thus affecting the conversion from SNR to illuminance discussed in Section 4.2.4. Figure 5.11 shows the simulated performance of the 4, 8 and 16-CSK constellations shown in Figures 5.2, 5.3, and 5.4 against the experimentally measured illuminance. Each subfigure is partitioned into illuminance classes outlined in standard DIN 5035. Figure 5.11 (a) assumes no signal averaging is used after the ADC, Figure 5.11 (b) assumes 5 samples are averaged, and Figure 5.11 (c) assumes 20 samples are averaged.

An interesting observation is that when illuminance is considered, some constellations outperformed other constellations that they originally underperformed to when comparing them on an SNR basis. Specifically, the 8-CSK constellation optimized for a CCT of 3000K performed $1.5 \mathrm{~dB}$ worse than the IEEE 16-CSK when compared on an SNR basis, but performed relatively better when compared on an illuminance basis, requiring approximately $10 \mathrm{~lx}$ less light power in Figure 5.11a. This occurs because silicon photodidoes have a stronger response to red light than green and blue light. As the majority of the illuminance provided by a $3000 \mathrm{~K}$ constellation comes form the red LED, these constellations will produce a higher SNR than those of equal luminance with a smaller red LED contribution. Therefore, $3000 \mathrm{~K}$ constellations appear relatively worse to constellations not optimized for a specific CCT when being compared with equal SNR as opposed to equal illuminance.

Another noteworthy observation is that all the 4-CSK constellations in Figure 5.11 lie in the region classified for public areas and dark surroundings" by standard DIN 5035. The acuity of the human eye to color at these illumination levels is poor, 

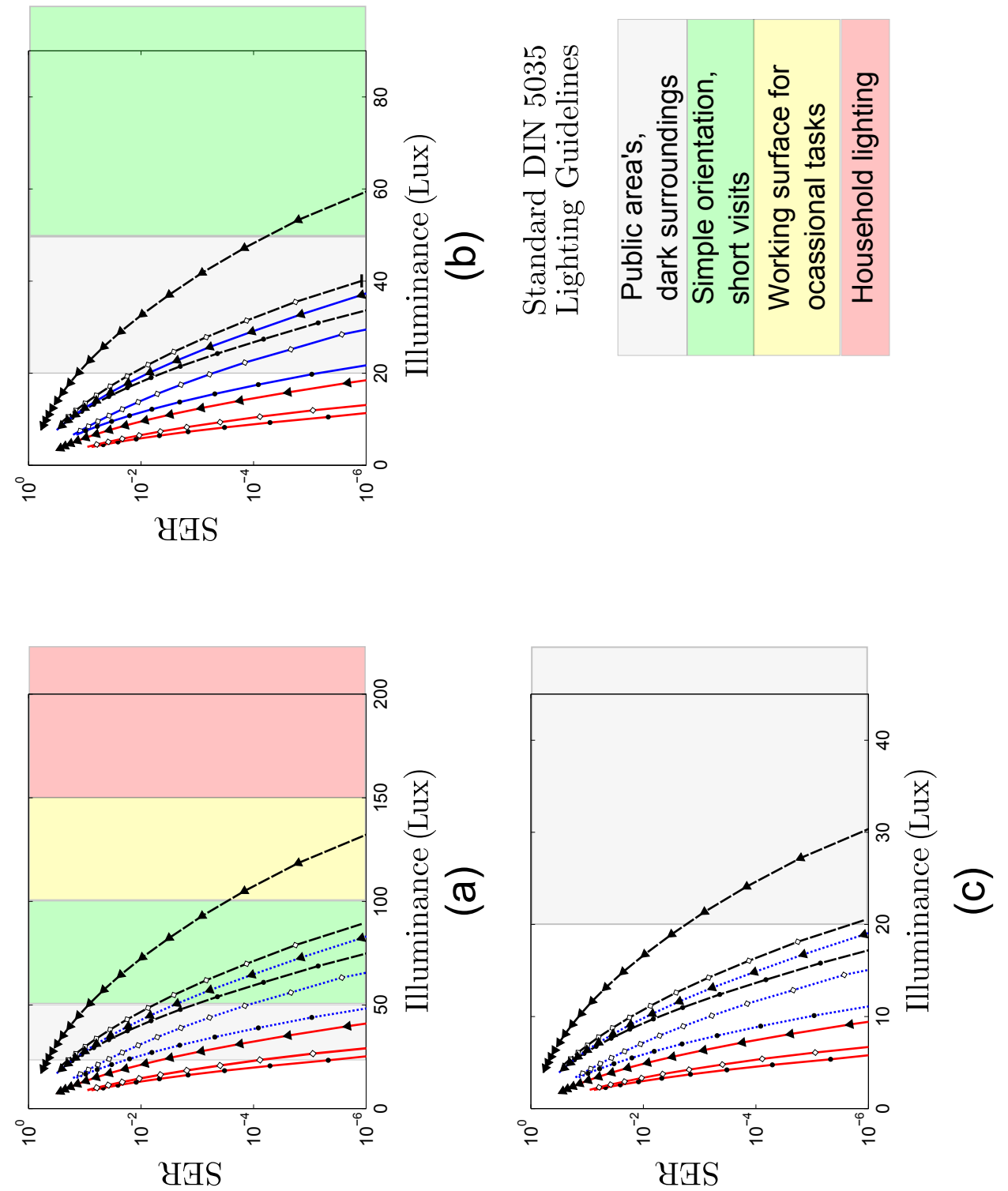

Figure 5.11: SER versus experimental Illuminance for various constellations 
suggesting there is no need to specify an operating color for the light source. Therefore, under low illuminance situations, the SER versus SNR can indeed be improved by removing the color constraint. Coupled with the limited $3 \mathrm{MHz}$ bandwidth of the LEDs, this confirms the need for high order, highly spectrally efficient symbol sets that take full advantage of the high SNR available in VLC channels.

\subsection{Conclusions}

In this chapter, the SER of 4, 8 and 16-CSK constellations, with and without a specified CCT, were both simulated and measured experimentally. It was shown the constellations using the interior point design method outlined in Chapter 3 consistently out performed the standardized IEEE design rules outlined in Section 2.6, thus validating the assumptions made about the channel model. Additionally, the need for optimizing constellations for a specific CCT was demonstrated. However, it was shown that varying the output color of the light source has a significant impact on error performance, with a $5 \mathrm{~dB}$ performance difference between a CCT of $2700 \mathrm{~K}$ and $6500 \mathrm{~K}$.

The chapter concluded with the simulation of the SER, of each constellation, versus illuminance. While the SNR is simpler to work with, as it reported after the matched, illuminance represents the real constraints imposed on VLC by the lighting industry. It was observed that the CCT of the constellation significantly affected the performance of a constellation when compared based on equal illuminance instead of equal SNR. This suggests that there is a relevant difference in comparing CSK constellations based on SNR or illuminance, and that future comparisons between VLC modulation formats should consider reporting SER versus illuminance. 


\section{Chapter 6}

\section{Concluding Remarks and Future Directions}

\subsection{Conclusion}

The primary contribution of this work was the CSK constellation design method, for arbitrarily sized constellations, presented in Chapter 3. The method, based on convex optimization techniques, was shown to optimize two dimensional and three dimensional CSK constellations while accounting for cross talk in the channel gain matrix. Additionally, the proposed method incorporated the study of colorimetry and was able to optimize constellations with a desired operating color in order to meet lighting industry illumination standards. Furthermore, a simple heuristic was provided for deriving constellations for large symbol sets with no specified operating color. The second significant contribution of this work is the first documented implementation of a CSK compatible VLC link. It was shown that the transmitter driver maintained strong linearity over a $200 \mathrm{~mA}$ dynamic range, but degraded for 
higher currents due to the non linearity of the LEDs themselves. Additionally, the bandwidth of the system was shown to be limited to approximately $3 \mathrm{MHz}$ by the LEDs, as the analog driver itself maintained a bandwidth of $10 \mathrm{MHz}$.

The primary results of this work arose from the application of the proposed design method for two dimensional CSK constellation when applied to the implemented VLC link. The VLC link was used to determine the SER versus SNR of 4, 8 and 16-CSK constellations, designed with the proposed methods, and compared their performance to the IEEE 802.15.7 standardized constellations. When the light source did not have its operating color constrained, the optimization technique produced constellations with a $3 \mathrm{~dB}$ improvement, in the experimental link, over the standardized IEEE constellations. While the IEEE standardized design rules are simple and do not require unique optimization based on the LEDs used, the results of this work clearly demonstrated that significant improvement can be made when the constellation are optimized. Furthermore, the need for optimizing constellations for a specific CCT was demonstrated as the IEEE constellations produced light colors inappropriate, according to Energy Star standards, for home and office lighting.

\subsection{Future Directions}

\subsubsection{Mobile Receiver Implementation and Characterization}

In Chapter 4 a CSK channel was implemented, and the available SNR against illuminance was measured. However, the test bed contained a high speed, precision instrumentation amplified photodetector with a large detector area and one inch aperture lens for gathering light. While the test bed was useful in determining the 


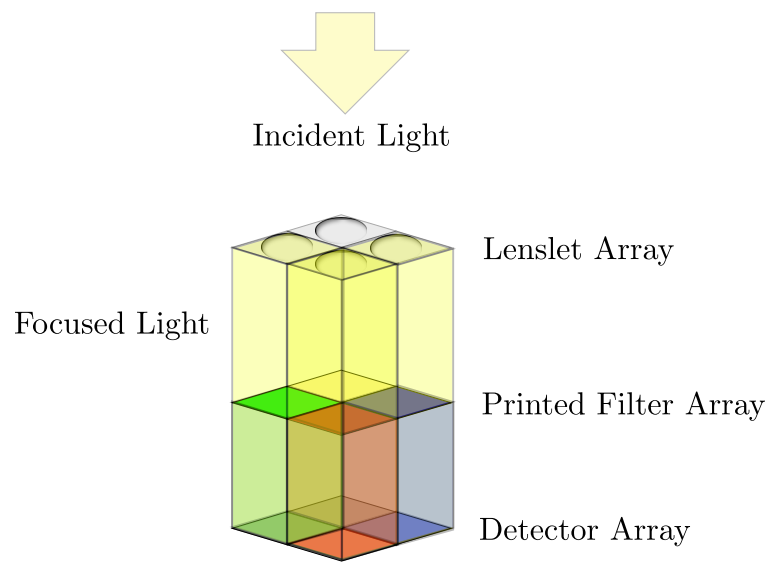

Figure 6.1: Simplified structure of a tri-chromatic ambient light sensor

effectiveness of the constellation design methods discussed in Chapter 3, it does not represent the SNR available to a typical end user, such as a mobile device. The direction under consideration is the measurement of SNR versus illumination, as well as noise figures, for a receiver designed for mobile devices in order to gain a perspective on the usefulness of CSK in a practical scenario.

Figure 6.1 depicts the simplified structure of a typical trichromatic ambient light sensor, available on the market, that could be used to enable CSK in mobile devices $[104,105]$. The typical trichromatic ambient light sensor consists of a rectangular silicon photodetector array with printed color filters for red, green, blue, and white light as well as an optical concentrator. As these devices have the same form factor, and similar cost and supporting circuitry as the monochromatic ambient light sensors used in current mobile devices, they are being explored for use in mobile devices, including tablets, to enable new features [105]. These features include,

1. Determining the type of light present (i.e. are you indoors or outdoors)

2. Tune color pallet of display based on ambient light color for better quality 
images

3. Real time white adjustment for video and photo capture

4. Chromatic adaption for mobile projectors

However, the detector area, per color, and concentrator lens diameter are typically $0.047 \mathrm{~mm}^{2}$ and $0.376 \mathrm{~mm}$ respectively; significantly smaller than the $12.96 \mathrm{~mm}^{2}$ detector area and 1 inch lens diameter described in Chapter 4.

\subsubsection{Adaption for Color Rendering}

The design methods outline in Chapter 3 were capable of compensating for color cross talk and allowed the chromaticity of the source to be specified. However, the notion of color rendering, defined in Section 2.2, was overlooked as the CRI was found to be a poor indicator of the color rendering ability of LED light sources. The new color rendering metric under study, color quality scale (CQS), has received attention as the potential successor to CRI as studies suggest it can accurately quantify the color rendering ability of any light source. The direction under consideration is adapting the algorithms in Chapter 3 to optimize constellations over the CQS. 


\section{References}

[1] G. Doe. (2013) The National Broadband Plan. Federal Communications Commission. [Online]. Available: http://download.broadband.gov/plan/ national-broadband-plan.pdf (Accessed: 23 September 2013)

[2] Cisco Systems Inc. (2013, Feb.) Cisco Visual Networking Index: Global Mobile Data Traffic Forecast Update, 2012 to 2017. [Online]. Available: http://www.cisco.com/en/US/solutions/collateral/ns341/ns525/ns537/ ns705/ns827/white_paper_c11-520862.html (Accessed: 23 September 2013)

[3] V. Chandrasekhar, J. Andrews, and A. Gatherer, "Femtocell Networks: A Survey," IEEE Commun. Mag., vol. 46, no. 9, pp. 59-67, 2008.

[4] P. Palanisamy and S. Nirmala, "Downlink interference management in femtocell networks - a comprehensive study and survey," in Proc. International Conference on Information Communication and Embedded Systems, Amphawa, (ICICES). Thailand, 2013, pp. 747-754.

[5] N. Saquib, E. Hossain, L. B. Le, and D. I. Kim, "Interference management in OFDMA femtocell networks: issues and approaches," IEEE Trans. Wireless Commun., vol. 19, no. 3, pp. 86-95, 2012. 
[6] A. Mahdy and J. Deogun, "Wireless optical communications: a survey," in Proc. IEEE Wireless Communications and Networking Conference, vol. 4, Atlanta, (WCNC). Georgia, 2004, pp. 2399-2404 Vol.4.

[7] L. Matabishi, "Theoretical implementation of antenna polarization to improve the reduction of co-channel interference in mobile cellular systems," in Proc. IEEE International Symposium on Microwave, Antenna, Propagation and EMC Technologies for Wireless Communications, vol. 1, Beijing, (MAPE). China, 2005, pp. 431-433 Vol. 1.

[8] H. Zhang, D. ru Pan, and X. Dai, "Iterative intercarrier interference reduction for mobile OFDM systems," in Proc. International Conference on Wireless Communications and Signal Processing, Suzhou, (WCSP). China, 2010, pp. $1-4$.

[9] M. Liang, D. C. Yang, and Y. F. Wang, "Spectrum reuse schemes for OFDMA cellular networks with fixed relay stations," in Proc. IET 2nd International Conference on Wireless, Mobile and Multimedia Networks, Beijing, (ICWMMN). China, 2008, pp. 394-397.

[10] N. Baldo, A. Asterjadhi, and M. Zorzi, "Cooperative Detection and Spectrum Reuse using a Network Coded Cognitive Control Channel," in Proc. 6th Annual IEEE Communications Society Conference on Sensor, Mesh and Ad Hoc Communications and Networks Workshops, Rome, (SECON). Italy, 2009, pp. $1-7$.

[11] W.-J. Chang, J.-W. Lim, H. K. Ahn, H. Kim, and H.-K. Yu, "60 GHz Amplifier 
MMICs and Module for $60 \mathrm{GHz}$ WPAN System," in Proc. IEEE Radio and Wireless Symposium, Long Beach, (RWS). California, 2007, pp. 377-380.

[12] D. O'Brien, L. Zeng, H. Le-Minh, G. Faulkner, J. Walewski, and S. Randel, "Visible light communications: Challenges and possibilities," in Proc. IEEE 19th International Symposium on Personal, Indoor and Mobile Radio Communications, Cannes, (PIMRC). France, 2008, pp. 1-5.

[13] T. Komine and M. Nakagawa, "Integrated system of white LED visible-light communication and power-line communication," IEEE Trans. Consum. Electron., vol. 49, no. 1, pp. 71-79, 2003.

[14] European Commission. Project hOME Gigabit Access. [Online]. Available: http://www.ict-omega.eu/ (Accessed: 23 September 2013)

[15] Byte Light. Indoor Location Based Software. [Online]. Available: http: //www.bytelight.com/ (Accessed: 23 September 2013)

[16] D. Steigerwald, J. Bhat, D. Collins, R. M. Fletcher, M. Holcomb, M. Ludowise, P. Martin, and S. Rudaz, "Illumination with solid state lighting technology," IEEE J. Sel. Topics. Quantum Electron., vol. 8, no. 2, pp. 310-320, 2002.

[17] J. N. Holonyak, "Is the light emitting diode (LED) an ultimate lamp?" J. Phys, vol. 68 , pp. 864-866, 2000.

[18] Natural Resources Canada. (2011, December) Phase-Out of Inefficient Light Bulbs. [Online]. Available: http://oee.nrcan.gc.ca/regulations/17725 (Accessed: 23 September 2013) 
[19] Department of Natural Resources. (2011, April) Regulations Amending the Energy Efficiency Regulations. [Online]. Available: http://www.gazette.gc. ca/rp-pr/p1/2011/2011-04-16/html/reg1-eng.html (Accessed: 23 September 2013)

[20] Visible Light Communication Consortium. VLCC: Home. [Online]. Available: http://www.vlcc.net (Accessed: 23 September 2013)

[21] P. Amirshahi and M. Kavehrad, "Broadband access over medium and low voltage power-lines and use of white light emitting diodes for indoor communications," in Proc. 3rd IEEE Consumer Communications and Networking Conference, Las Vegas, (CCNC). Nevada, 2006, pp. 897-901.

[22] pureVLC. Delivering data through light. [Online]. Available: http: //purevlc.co.uk/ (Accessed: 23 September 2013)

[23] Boston University. Smart Lighting Engineering Research Center. [Online]. Available: http://www.bu.edu/smartlighting/ (Accessed: 23 September 2013)

[24] IEEE Standard for Local and Metropolitan Area Networks-Part 15.7: ShortRange Wireless Optical Communication Using Visible Light, IEEE Std. 802.15.7, 2011.

[25] A. Wilkins, J. Veitch, and B. Lehman, "LED lighting flicker and potential health concerns: IEEE standard PAR1789 update," in Proc. IEEE Energy Conversion Congress and Exposition, Atlanta, (ECCE). Georgia, 2010, pp. 171-178.

[26] Visible Light Communication System Standard, Japan Electronics and Information Technology Industries Association (JEITA) Std. CP-1221, 2007. 
[27] Visible Light ID System Standard, Japan Electronics and Information Technology Industries Association (JEITA) Std. CP-1222, 2007.

[28] J. Grubor, S. Randel, K.-D. Langer, and J. Walewski, "Broadband Information Broadcasting Using LED-Based Interior Lighting," J. Lightw. Technol., vol. 26, no. 24 , pp. 3883-3892, 2008.

[29] J. Vucic, J., C. Kottke, S. Nerreter, A. Buttner, K.-D. Langer, and J. Walewski, "White Light Wireless Transmission at $200 \mathrm{Mb} / \mathrm{s}$ Net Data Rate by Use of Discrete-Multitone Modulation," IEEE Photonics Technol. Lett., vol. 21, no. 20, pp. 1511-1513, 2009.

[30] J. Vucic, C. Kottke, S. Nerreter, K. Habel, A. Buttner, K.-D. Langer, and J. Walewski, "125 Mbit/s over $5 \mathrm{~m}$ wireless distance by use of OOK-Modulated phosphorescent white LEDs," in Proc. 35th European Conference on Optical Communication, Vienna, (ECOC). Austria, 2009, pp. 1-2.

[31] P. Butala, J. Chau, and T. D. C. Little, "Metameric modulation for diffuse visible light communications with constant ambient lighting," in Proc. International Workshop on Optical Wireless Communications, Pisa, (IWOW) . Italy, 2012, pp. 1-3.

[32] A. Keppens and Y. Zong, "Efficiency Evaluation and Comparison of Phosphor White and RGB packages for high power LED applications," in Proc. 27th Session of the CIE, vol. 37, 2011. 
[33] C. Wetzel and T. Detchprohm, "Wavelength-stable rare earth-free green lightemitting diodes for energy efficiency," Opt Express, vol. 19 Suppl 4, pp. A962971, Jul 2011.

[34] Z.-P. Yang and X.-N. Li, "A Novel Green-Emitting Phosphor BaZnP/sub 2/O/sub 7/:Tb/sup 3+/ for White Leds and the Infuence of Doping Ce/sup," in Proc. Symposium on Photonics and Optoelectronics, Wuhan, (SOPO). China, 2009, pp. 1-3.

[35] Cree Incorporated. CREE Technical Report: Eye Safety With LED Components. [Online]. Available: http://www.cree.com/ /media/Files/Cree/ LED\%20Components\%20and\%20Modules/XLamp/XLamp\%20Application\% 20Notes/XLamp_EyeSafety.pdf (Accessed: 23 September 2013)

[36] Philips Lumileds Lighting Company. Philips Lighting. [Online]. Available: http://www.lighting.philips.ca/en/ (Accessed: 23 September 2013)

[37] Philips. hue: Personal Wireless Lighting. [Online]. Available: https: //www.meethue.com/en-CA (Accessed: 23 September 2013)

[38] J. Hou and D. O'Brien, "Vertical handover-decision-making algorithm using fuzzy logic for the integrated Radio-and-OW system," IEEE Trans. Wireless Commun., vol. 5, no. 1, pp. 176-185, 2006.

[39] S. Alavi, A. Supa'at, and S. Idrus, "Integrated system of visible free space optic with PLC," in IEEE 9th Malaysia International Conference on Communications, Kuala Lumpur, (MICC). Malaysia, 2009, pp. 271-275. 
[40] S. Alavi, H. Rezaie, and A. Supa'at, "Application of OFDM on integrated system of visible free space optic with PLC," in Proc. IEEE Asia-Pacific Conference on Applied Electromagnetics, Port Dickson, (APACE). Malaysia, 2010, pp. $1-5$.

[41] L. Zeng, D. O'Brien, H. Minh, G. Faulkner, K. Lee, D. Jung, Y. Oh, and E. T. Won, "High data rate multiple input multiple output (MIMO) optical wireless communications using white led lighting," IEEE J. Sel. Areas Commun., vol. 27, no. 9, pp. 1654-1662, 2009.

[42] K. Izumi, "Trend of seamless positioning technology," Photogrammetry and Remote Sensing, vol. 49, pp. 41-44, 2010.

[43] S. H. Michael Taylor, "Angular Diversity Approach to Indoor Positioning Using Visible Light," in Proc. IEEE Globecom, Atlanta, GA, Dec Atlanta, (GC Wkshps). Georgia, 2013.

[44] Audi AG. Defeat Darkness. [Online]. Available: http://www.audileds.com (Accessed: 23 September 2013)

[45] G. Pang, T. Kwan, C.-H. Chan, and H. Liu, "LED traffic light as a communications device," in Proc. IEEE/IEEJ/JSAI International Conference on Intelligent Transportation Systems, Tokyo. Japan, 1999, pp. 788-793.

[46] K. Deeg. (2009, June) Green Means Go: Energy-Efficient LED Traffic Signals in Winnipeg. National Resources Canada. [Online]. Available: http: //oee.nrcan.gc.ca/equipment/ready-articles/10721 (Accessed: 23 September 2013) 
[47] R. Drost and B. Sadler, "Constellation design for color-shift keying using billiards algorithms," in Proc. IEEE GLOBECOM Workshops, Miami, (GC Wkshps). Florida, 2010, pp. 980-984.

[48] B. Bai, Q. He, Z. Xu, and Y. Fan, "The color shift key modulation with nonuniform signaling for visible light communication," in Proc. 1st IEEE International Conference on Communications in China Workshops, Beijing, (ICCC). China, 2012, pp. 37-42.

[49] A. Valberg, Light Vision Color. Chichester, WSX: Wiley, 2005.

[50] S. Nundy and D. Purves, "A probabilistic explanation of brightness scaling," in Proc. National Academy of Sciences of the United States of America, vol. 99, no. 22, October 2002, pp. 14482-14487.

[51] Cree Incorporated. CREE Technical Report: LED Color Mixing, Basics and Background. [Online]. Available: http://www.cree.com/ /media/Files/Cree/LED\%20Components\%20and\% 20Modules/XLamp/XLamp\%20Application\%20Notes/LED_color_mixing.pdf (Accessed: 23 September 2013)

[52] CIE: International Commission on Illumination. ISO 11664 / CIE S 014. [Online]. Available: http://www.cie.co.at/Publications/Standards (Accessed: 23 September 2013)

[53] Vishay Intertechnology, Inc. Vishay: LED Products. [Online]. Available: http://www.vishay.com/leds/ (Accessed: 23 September 2013) 
[54] OSRAM Licht AG. OSRAM: LED Solutions. [Online]. Available: $\quad$ http://ledlight.osram-os.com/farther/?gclid= CLC34Lm3lLcCFYMWMgod9GEAgA (Accessed: 23 September 2013)

[55] S. Stevens, Psychophysics. New Brunswick, NJ: Wiley, 2008.

[56] Canadian Center for Occupational Health and Safety. Lighting Ergonomics - Light Flicker. [Online]. Available: http://www.ccohs.ca/oshanswers/ ergonomics/lighting_flicker.html (Accessed: 23 September 2013)

[57] C. Schneider, "Electrophysiological analysis of the mechanisms underlying critical flicker frequency," Vision Research, vol. 8, pp. 1235-1244, 1968.

[58] S. Hecht and S. Shlaer, "Intermittent Stimulation by Light: V. The Relation Between Intensity and Critical Frequency for Different Parts of the Spectrum," The Journal of General Physiology, vol. 19, no. 6, pp. 965-977, 1936. [Online]. Available: http://jgp.rupress.org/content/19/6/965.abstract (Accessed: 23 September 2013)

[59] S. Veitch, J.A.; McColl, "Modulation of fluorescent light: flicker rate and light source effects on visual performance and visual comfort," Lighting Research and Technology, vol. 27, pp. 243-256, 1995.

[60] Electromagnetic compatibility, International Electrotechnical Commission (IEC) Std. IEC $61000,2013$.

[61] Canadian Center for Occupational Health and Safety. Consilidation of Canada Occupational Health and Safety Regulations. [Online]. Available: 
http://laws-lois.justice.gc.ca/PDF/SOR-86-304.pdf (Accessed: 23 September 2013)

[62] Artificial lighting of interiors, DIN Std. 5035, 1990.

[63] ENERGY STAR. ENERGY STAR Program Requirements for Integral LED Lamps. [Online]. Available: http://www.energystar.gov/ia/partners/product_ specs/program_reqs/Integral_LED_Lamps_Program_Requirements.pdf （Accessed: 23 September 2013)

[64] ETAP Lighting. EN12464-1 : brief description of the standard. [Online]. Available: http://www.etaplighting.com/uploadedFiles/ Downloadable_documentation/documentatie/EN12464_E_OK.pdf (Accessed: 23 September 2013)

[65] Photobiological safety of lamps and lamp systems, International Electrotechnical Commission (IEC) Std. IEC 62 471, 2006.

[66] Safety of laser products, International Electrotechnical Commission (IEC) Std. IEC $60825,2007$.

[67] CIE Division 2: Physical Measurement of Light and Radiation. Calculating CRI. Calculating\%20CRI-CAM02UCS-v2.xls? [Online]. Available: cie2.nist. gov/TC1-69/Leeds/ (Accessed: 23 September 2013)

[68] X. Guo, "A review of colour rendering indices and their application to commercial light sources," Lighting Research and Technology, vol. 36, pp. 183-197, 2004. 
[69] W. Davis and Y. Ohno. Color Rendering of Light Sources. National Institute of Standards and Technology. [Online]. Available: http://web.archive.org/web/20090825230627/http://physics.nist.gov/ Divisions/Div844/facilities/vision/color.html (Accessed: 23 September 2013)

[70] I. Ashdown. (2008, January) Color Rendering Index and LEDs. U.S. Department of Energy. [Online]. Available: http://cool.conservation-us.org/ byorg/us-doe/color_rendering_index.pdf (Accessed: 23 September 2013)

[71] G. Agrawal, Fiber-Optic Communication Systems, K. Chang, Ed. New York, NY: Wiley, 2002.

[72] T. Komine and M. Nakagawa, "Fundamental analysis for visible-light communication system using LED lights," IEEE Trans. Consum. Electron., vol. 50, no. 1, pp. 100-107, 2004.

[73] W. Chewpraditkul, L. Swiderski, M. Moszynski, T. Szczesniak, A. SyntfeldKazuch, C. Wanarak, and P. Limsuwan, "Scintillation Properties of LuAG:Ce, YAG:Ce and LYSO:Ce Crystals for Gamma-Ray Detection," IEEE Trans. Nucl. Sci., vol. 56, no. 6, pp. 3800-3805, 2009.

[74] M. I. Yoshikazu Matsuia, Hiroshi Horikawab and W. Parkc, "Preparation of YAG : Ce nanocrystals by an environmentally friendly wet process Effect of Ce3+ concentration on photoluminescent property," Journal of Ceramic Processing Research, vol. 12, no. 3, pp. 348-351, 2011.

[75] D. S. Meyaard, G.-B. Lin, J. Cho, E. Fred Schubert, H. Shim, S.-H. Han, M.-H. Kim, C. Sone, and Y. Sun Kim, "Identifying the cause of the efficiency droop 
in GaInN light-emitting diodes by correlating the onset of high injection with the onset of the efficiency droop," Applied Physics Letters, vol. 102, no. 25, pp. 251114-251114-4, 2013.

[76] J. Kahn and J. Barry, "Wireless infrared communications," Proc. IEEE, vol. 85, no. 2, pp. 265-298, 1997.

[77] V. Jungnickel, V. Pohl, S. Nonnig, and C. Von Helmolt, "A physical model of the wireless infrared communication channel," IEEE J. Sel. Areas Commun., vol. 20, no. 3, pp. 631-640, 2002.

[78] D. Rogers, "Integrated optical receivers using MSM detectors," J. Lightw. Technol., vol. 9, no. 12, pp. 1635-1638, 1991.

[79] S. Dimitrov and H. Haas, "Information Rate of OFDM-Based Optical Wireless Communication Systems With Nonlinear Distortion," J. Lightw. Technol., vol. 31, no. 6, pp. 918-929, 2013.

[80] X. Li, R. Mardling, and J. Armstrong, "Channel Capacity of IM/DD Optical Communication Systems and of ACO-OFDM," in Proc. IEEE International Conference on Communications, Glasgow, (ICC). Scotland, 2007, pp. 21282133.

[81] S. Hussin, K. Puntsri, and R. Noe, "Performance analysis of optical OFDM systems," in Proc. 3rd International Congress on Ultra Modern Telecommunications and Control Systems and Workshops, Budapest, (ICUMT). Hungary, 2011, pp. 1-5. 
[82] R. Gohary and T. Davidson, "Noncoherent MIMO communication: Grassmannian constellations and efficient detection," in Proc. International Symposium on Information Theory, Chicago, (ISIT). Illinois, 2004, pp. 65-.

[83] S. Boyd and L. Vandenberghe, Convex Optimization. Cambridge University Press, 2004.

[84] MATLAB, version 7.10.0 (R2010a). Natick, Massachusetts: The MathWorks Inc., 2010.

[85] H. J. B. M. Melissen, "Densest packings of congruent circles in an equilateral triangle," The American Mathematical Monthly, vol. 100, pp. 916-925, 1993.

[86] Terasic Technologies. DE2-70 User Manual. [Online]. Available: ftp://ftp.altera.com/up/pub/Altera_Material/12.1/Boards/DE2-70/ DE2_70_User_Manual.pdf (Accessed: 23 September 2013)

[87] Analog Devices. CMOS, 330 MHzTriple 10-Bit High Speed Video DAC. [Online]. Available: http://www.analog.com/static/imported-files/data_sheets/ ADV7123.pdf (Accessed: 23 September 2013)

[88] Altera. Cyclone II Device Handbook, Volume 1. [Online]. Available: http:// www.altera.com/literature/hb/cyc2/cyc2_cii5v1.pdf (Accessed: 23 September 2013)

[89] Linear Technology. LT 1365 Quad 70MHz, 1000V/s Op Amp. [Online]. Available: http://cds.linear.com/docs/en/datasheet/13645fa.pdf (Accessed: 23 September 2013) 
[90] Panasonic Corporation. 2SD2358 Silicon NPN epitaxial planar type. [Online]. Available: http://www.semicon.panasonic.co.jp/ds4/2SD2358_BED_discon.pdf (Accessed: 23 September 2013)

[91] Philips Lumileds Lighting Company. LUXEON Rebel and LUXEON Rebel ES Color Portfolio. [Online]. Available: http://www.luxeonstar.com/v/vspfiles/ downloadables/DS68.pdf (Accessed: 23 September 2013)

[92] Luxeon Star LEDs. Predefined Tri-Star LED. [Online]. Available: http://www. luxeonstar.com/Predfined-Tri-Star-LED-Assembly-p/sr-03-000132.htm (Accessed: 23 September 2013)

[93] ABRA Electronics. AB-330CUL DC Power Supply 30V/3A+30V/3A+5V/3A Triple output. [Online]. Available: http://www.abra-electronics.com/products/ AB\%252d330CUL-DC-Power-Supply-30V\%7B47\%7D3A\%252b30V\%7B47\% 7D3A\%252b5V\%7B47\%7D3A-Triple-output.html (Accessed: 23 September 2013)

[94] Thorlabs Inc. (2012, March) PM 100 Optical Power Meter System Operation Manual. [Online]. Available: http://www.thorlabs.us/Thorcat/ 12200/PM100-Manual.pdf (Accessed: 23 September 2013)

[95] Thorlabs Inc. Spec Sheet: S121B Silicon Power Meter Optical Head. [Online]. Available: http://www.thorlabs.us/Thorcat/14100/S121B-SpecSheet.pdf (Accessed: 23 September 2013)

[96] Thorlabs Inc. PDA36A Si Switchable Gain Detector. [Online]. Available: 
http://www.thorlabs.us/Thorcat/13000/PDA36A-Manual.pdf (Accessed: 23 September 2013)

[97] LEE Filters. 024 Scarlet Polyester Filter. [Online]. Available: http://www. leefilters.com/lighting/colour-details.html\#024 (Accessed: 23 September 2013)

[98] LEE Filters. 738 JAS Green Polyester Filter. [Online]. Available: http://www. leefilters.com/lighting/colour-details.html\#738 (Accessed: 23 September 2013)

[99] LEE Filters. 079 Just Blue Polyester Filter. [Online]. Available: http://www. leefilters.com/lighting/colour-details.html\#079 (Accessed: 23 September 2013)

[100] Agilent Technologies. Infiniium 54850 Series Oscilloscopes and InfiniiMax 1130 Series Probes Data Sheets. [Online]. Available: http://cp.literature.agilent. com/litweb/pdf/5988-7976EN.pdf (Accessed: 23 September 2013)

[101] S. Wilson, Digital Modulation and Coding, T. Robbins, Ed. Prentice Hall, 1996.

[102] R. Gray and D. Neuhoff, "Quantization," IEEE Trans. Inf. Theory, vol. 44, no. 6, pp. 2325-2383, 1998.

[103] Extech Instruments. HD400: Heavy Duty Light Meter Data Sheet. [Online]. Available: http://www.extech.com/instruments/resources/datasheets/HD400_ HD450.pdf (Accessed: 23 September 2013)

[104] Austria Micro Systems. TCS3103 - Light-To-Voltage Color Sensor. [Online]. Available: http://www.ams.com/eng/Products/Light-Sensors/Color-Sensor/ TCS3103 (Accessed: 23 September 2013) 
[105] Maxim Integrated Products Inc. Maxim Mobile Sensor: Today and Tomorrow. [Online]. Available: http://www.wca.org/event_archives/2012/ Maxim_WCA_April2012_Mobile_Sensors.pdf (Accessed: 23 September 2013) 


\section{Appendix A}

\section{Designed Constellations}

This Appendix contains the numeric representation of the constellation examples shown in Sections 3.5, 3.6 and 3.7.2. Furthermore, this Appendix contains the digital representations, use by the digital-to-analog converters, of all constellations designed in Section 5.1.2.

\section{A.1 Constellations from Chapter 3}

This section contains the numeric representation of all constellations examples described in Chapter 3. The figure identification number is listed along side its corresponding data. 


\begin{tabular}{r|r|r|l|r|r|} 
& \multicolumn{1}{|c|}{$\mathrm{x}$} & $\mathrm{y}$ & & $\mathrm{x}$ & $\mathrm{y}$ \\
\hline Figure 3.2 (a) & 0 & -0.408 & Figure 3.2 (b) & 0 & 0 \\
-0.707 & -0.408 & & 0.236 & -0.408 \\
0.707 & -0.408 & & -0.707 & -0.408 \\
0.354 & -0.076 & & 0.707 & -0.408 \\
0 & 0.816 & & 0.236 & 0.408 \\
0.243 & 0.396 & & 0 & 0.816 \\
& -0.354 & -0.076 & & -0.236 & 0.408 \\
& -0.243 & 0.396 & & -0.236 & -0.408
\end{tabular}

\begin{tabular}{r|r|r|l|r|r|} 
& \multicolumn{1}{|c|}{$\mathrm{x}$} & \multicolumn{1}{c|}{$\mathrm{y}$} & & $\mathrm{x}$ & \multicolumn{1}{c|}{$\mathrm{y}$} \\
\hline Figure 3.4 (a) & 0.147 & 0.055 & Figure 3.4 (b) & 0.022 & 0.167 \\
& -0.447 & -0.238 & & 0.446 & 0.045 \\
-0.207 & -0.408 & & 0.174 & -0.101 \\
& 0.44 & 0.055 & & -0.118 & -0.408 \\
0.698 & -0.392 & & 0 & 0.816 \\
0 & 0.309 & & -0.207 & 0.458 \\
& 0.447 & -0.238 & & -0.133 & -0.103 \\
-0.44 & 0.055 & & -0.413 & -0.34 \\
& -0.698 & -0.392 & & -0.283 & 0.164 \\
-0.146 & 0.564 & & 0.445 & -0.257 \\
0.207 & -0.408 & & 0.294 & 0.306 \\
& 0 & -0.2 & & -0.5 & -0.049 \\
& 0.146 & 0.564 & & 0.088 & 0.527 \\
& -0.147 & 0.055 & & -0.707 & -0.408 \\
& -0.293 & 0.31 & & 0.707 & -0.408 \\
& 0.293 & 0.31 & & 0.184 & -0.408
\end{tabular}

\begin{tabular}{|c|c|c|c|c|c|}
\hline Figure 3.3 (a) & -0.396 & 0.131 & Figure 3.3 (b) & -0.178 & -0.203 \\
\hline & 0.394 & 0.133 & & -0.206 & 0.459 \\
\hline & 0.236 & 0.133 & & 0.206 & 0.46 \\
\hline & -0.158 & 0.543 & & 0.003 & -0.104 \\
\hline & -0.08 & -0.144 & & -0.206 & 0.254 \\
\hline & -0.318 & -0.007 & & 0 & 0.254 \\
\hline & -0.477 & -0.285 & & 0.59 & -0.205 \\
\hline & -0.001 & 0.544 & & 0.177 & -0.215 \\
\hline & -0.16 & -0.006 & & 0.28 & -0.033 \\
\hline & -0.636 & -0.286 & & -0.281 & -0.026 \\
\hline & -0.398 & -0.146 & & -0.102 & 0.075 \\
\hline & -0.079 & 0.68 & & 0 & 0.816 \\
\hline & 0.315 & 0.271 & & -0.384 & 0.152 \\
\hline & -0.237 & 0.406 & & 0.707 & -0.408 \\
\hline & -0.08 & 0.407 & & 0.101 & -0.408 \\
\hline & -0.001 & -0.005 & & 0.384 & 0.152 \\
\hline & -0.159 & 0.27 & & 0.487 & -0.027 \\
\hline & -0.556 & -0.146 & & 0.103 & 0.638 \\
\hline & 0.158 & -0.006 & & -0.707 & -0.408 \\
\hline & -0.081 & 0.132 & & 0.105 & 0.076 \\
\hline & -0.001 & 0.27 & & -0.101 & -0.393 \\
\hline & -0.239 & -0.145 & & -0.487 & -0.028 \\
\hline & 0.236 & 0.408 & & -0.505 & -0.392 \\
\hline & 0.157 & 0.271 & & -0.384 & -0.209 \\
\hline & 0.157 & 0.545 & & 0.505 & -0.391 \\
\hline & -0.238 & 0.132 & & 0.383 & -0.214 \\
\hline & -0.316 & 0.269 & & -0.59 & -0.206 \\
\hline & 0.077 & 0.133 & & 0 & 0.459 \\
\hline & -0.476 & -0.008 & & 0.206 & 0.255 \\
\hline & & 0.816 & & 0.303 & -0.408 \\
\hline & 0.078 & 0.681 & & -0.303 & -0.408 \\
\hline & 0.078 & 0.408 & & -0.103 & 0.638 \\
\hline
\end{tabular}

\begin{tabular}{|l|l|l|}
\hline Figure $3.5(\mathrm{a})$ & -0.202 & 0.179 \\
\hline
\end{tabular} $0.418-0.056$ $0.037-0.267$ \begin{tabular}{ll|l}
0.184 & 0.38
\end{tabular} 0.0670 .598 $-0.082-0.044$ $-0.071 \quad 0.391$ $-0.683-0.368$ $0.535-0.275$

$0.169-0.052$

$0.286-0.275$

$-0.213-0.259$

0.050 .169

0.3010 .162

$-0.333-0.034$

$|-0.463|-0.248$ 


\begin{tabular}{|c|c|c|c|c|c|c|c|c|c|c|c|c|c|c|c|c|}
\hline & $x$ & $y$ & \begin{tabular}{|l|l}
$x$ \\
\end{tabular} & $y$ & $\mathrm{x}$ & $y$ & \begin{tabular}{|l|l}
$x$ \\
\end{tabular} & \begin{tabular}{|l|}
$\mathrm{y}$ \\
$\mathrm{y}$
\end{tabular} & $\mathrm{x}$ & \begin{tabular}{ll|}
$y$ \\
$y$
\end{tabular} & $x$ & $y$ & $x$ & $y$ & $\mathrm{x}$ & $y$ \\
\hline \multirow{16}{*}{ Figure $3.6(\mathrm{a})$} & \begin{tabular}{|l|l|}
0.516 \\
\end{tabular} & -0.245 & \begin{tabular}{|l|}
0.328 \\
\end{tabular} & \begin{tabular}{|l|}
0.081 \\
\end{tabular} & \begin{tabular}{|l|}
-0.419 \\
\end{tabular} & -0.078 & \begin{tabular}{|l|}
0.076 \\
\end{tabular} & -0.326 & -0.096 & \begin{tabular}{|l|}
0.651 \\
\end{tabular} & -0.049 & 0.235 & -0.002 & \begin{tabular}{|l|}
0.487 \\
\end{tabular} & \begin{tabular}{|l|}
-0.707 \\
\end{tabular} & -0.408 \\
\hline & -0.192 & \begin{tabular}{|l|} 
\\
0.151
\end{tabular} & 0.045 & 0.405 & 0.14 & \begin{tabular}{|l|} 
\\
\end{tabular} & 0.185 & \begin{tabular}{|l|}
0.162 \\
\end{tabular} & 0.469 & $-0.164 \mid$ & -0.097 & 0.485 & -0.288 & 0.317 & -0.384 & 0.152 \\
\hline & 0.142 & 0.571 & -0.171 & $\mid-0.408$ & 0.045 & 0.24 & -0.096 & 0.151 & -0.34 & -0.026 & 0.188 & 0.325 & -0.145 & 0.234 & -0.048 & 0.734 \\
\hline & 0.092 & 0.324 & -0.467 & $|-0.161|$ & 0.322 & -0.246 & -0.247 & -0.192 & 0.236 & \begin{tabular}{|l|} 
\\
\end{tabular} & 0.189 & 0.489 & -0.018 & 0.005 & 0.174 & -0.326 \\
\hline & & 0.816 & -0.563 & \begin{tabular}{|l|}
-0.16 \\
\end{tabular} & -0.514 & -0.243 & -0.244 & $|-0.026|$ & -0.371 & $|-0.162|$ & -0.192 & 0.484 & -0.611 & $\mid-0.408$ & 0.422 & -0.082 \\
\hline & -0.24 & 0.234 & 0.03 & $|-0.078|$ & -0.562 & -0.326 & 0.139 & \begin{tabular}{|l|} 
\\
\end{tabular} & 0.046 & \begin{tabular}{|l|} 
\\
\end{tabular} & 0.047 & 0.734 & -0.015 & \begin{tabular}{|l|} 
\\
\end{tabular} & -0.022 & -0.326 \\
\hline & -0.516 & -0.077 & 0.33 & 0.245 & 0.227 & -0.082 & 0.125 & -0.408 & 0.235 & 0.244 & 0.078 & 0.005 & -0.049 & 0.569 & -0.468 & 0.006 \\
\hline & 0.028 & -0.244 & -0.388 & \begin{tabular}{|l|}
0.057 \\
\end{tabular} & 0.37 & $|-0.327|$ & 0.223 & $|-0.244|$ & -0.418 & $-0.245 \mid$ & 0.377 & 0.163 & 0.375 & $|-0.001|$ & -0.268 & -0.408 \\
\hline & -0.293 & $\mid-0.108$ & 0.274 & $|-0.164|$ & -0.144 & 0.567 & 0.027 & -0.408 & 0.325 & $|-0.083|$ & 0.611 & $\mid-0.408$ & 0.707 & $\mid-0.408$ & -0.003 & 0.321 \\
\hline & -0.514 & $\mid-0.408$ & -0.148 & $-0.027 \mid$ & -0.07 & -0.243 & -0.292 & 0.057 & 0.471 & 0 & -0.167 & $|-0.243|$ & -0.098 & 0.318 & -0.12 & -0.326 \\
\hline & -0.02 & $|-0.161|$ & -0.193 & \begin{tabular}{|l|}
0.317 \\
\end{tabular} & -0.116 & -0.16 & 0.564 & $|-0.327|$ & -0.415 & $|-0.408|$ & -0.067 & $-0.077 \mid$ & -0.342 & -0.346 & 0.42 & -0.246 \\
\hline & -0.197 & \begin{tabular}{|l|} 
\\
\end{tabular} & 0.278 & $|-0.001|$ & 0.077 & $-0.161 \mid$ & -0.24 & \begin{tabular}{|l|} 
\\
0.4
\end{tabular} & 0.518 & $|-0.081|$ & 0.18 & & 0.134 & 0.082 & 0.068 & 0.149 \\
\hline & 0.093 & 0.488 & -0.322 & $|-0.252|$ & -0.336 & 0.235 & 0.281 & 0.162 & -0.195 & 0.056 & 0.66 & -0.326 & 0.418 & -0.408 & 0.565 & -0.163 \\
\hline & -0.145 & 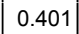 & -0.24 & $|-0.311|$ & 0.222 & -0.408 & -0.659 & -0.325 & -0.001 & \begin{tabular}{|l|} 
\\
\end{tabular} & 0.612 & $\mid-0.245$ & 0.094 & 0.653 & 0.32 & -0.408 \\
\hline & -0.611 & $|-0.243|$ & -0.288 & \begin{tabular}{|l|} 
\\
\end{tabular} & -0.071 & -0.408 & 0.126 & -0.079 & 0.125 & $|-0.244|$ & 0.515 & $\mid-0.408$ & -0.465 & -0.326 & -0.1 & 0.055 \\
\hline & -0.05 & 0.403 & 0.232 & 0.08 & 0.467 & -0.327 & 0.283 & 0.326 & 0.372 & $|-0.164|$ & 0.174 & $|-0.162|$ & 0.271 & $|-0.326|$ & 0.424 & 0.082 \\
\hline
\end{tabular}

\begin{tabular}{l|r|r|r|r|r|r|r|r|r|r|r|r|r|r|r|r|}
\hline Figure 3.6 (b) & 0.377 & -0.193 & -0.182 & -0.003 & 0.174 & 0.185 & -0.269 & 0.032 & 0.156 & -0.112 & -0.053 & 0.295 & 0.026 & -0.179 & 0.062 & 0.282 \\
& 0.089 & 0.235 & -0.085 & -0.047 & 0.271 & -0.201 & -0.321 & -0.261 & -0.039 & 0.195 & -0.191 & -0.201 & -0.186 & 0.132 & 0.242 & -0.155 \\
& 0.146 & 0.031 & 0.114 & -0.062 & 0.133 & -0.162 & -0.018 & 0.336 & -0.104 & -0.24 & 0.199 & 0.136 & -0.172 & 0.068 & -0.005 & -0.122 \\
-0.292 & -0.016 & 0.101 & 0.111 & 0.054 & 0.085 & -0.168 & -0.151 & 0.039 & 0.331 & 0.268 & -0.104 & -0.134 & -0.028 & 0.296 & -0.15 \\
& -0.353 & -0.219 & -0.114 & -0.147 & -0.254 & -0.114 & 0.266 & -0.01 & -0.401 & -0.13 & -0.31 & -0.119 & 0.101 & 0.058 & 0.164 & -0.208 \\
& 0.213 & -0.11 & -0.391 & -0.182 & -0.359 & -0.097 & 0.154 & 0.085 & 0.055 & -0.025 & -0.335 & -0.167 & 0.217 & -0.205 & -0.237 & -0.012 \\
& 0.187 & -0.158 & 0.009 & 0.169 & -0.222 & -0.157 & 0.007 & 0.058 & 0.102 & -0.116 & -0.028 & 0.247 & -0.057 & -0.141 & -0.05 & -0.234 \\
& 0.054 & 0.139 & 0.01 & -0.057 & -0.086 & 0.169 & -0.445 & -0.184 & -0.278 & -0.162 & -0.2 & -0.108 & -0.417 & -0.23 & 0.151 & -0.023 \\
& -0.136 & -0.196 & 0.102 & 0.001 & 0.007 & 0.112 & 0.224 & 0.089 & -0.233 & -0.065 & -0.039 & 0.03 & -0.335 & -0.049 & -0.04 & -0.08 \\
& 0.323 & -0.101 & 0.296 & -0.055 & 0.026 & 0.237 & -0.141 & 0.162 & -0.038 & 0.141 & -0.083 & 0.251 & 0.239 & -0.058 & 0.184 & -0.064 \\
& 0.146 & 0.139 & 0.072 & 0.374 & -0.267 & -0.255 & -0.082 & -0.19 & 0.015 & 0.379 & 0.004 & -0.229 & -0.506 & -0.275 & -0.136 & 0.029 \\
& 0.058 & -0.223 & 0.198 & 0.042 & 0.111 & -0.218 & 0.143 & 0.229 & -0.449 & -0.273 & -0.245 & -0.206 & -0.216 & 0.038 & -0.221 & 0.091 \\
& -0.087 & 0.006 & 0.08 & -0.173 & 0.062 & -0.078 & -0.377 & -0.267 & 0.057 & 0.192 & -0.027 & -0.185 & -0.145 & -0.099 & 0.122 & 0.279 \\
& 0.35 & -0.147 & -0.091 & -0.099 & 0.205 & -0.013 & 0.112 & 0.182 & 0.047 & 0.421 & -0.159 & -0.245 & 0.095 & 0.325 & -0.085 & 0.115 \\
& 0.055 & 0.03 & -0.138 & 0.108 & 0.324 & -0.197 & 0.006 & 0.288 & -0.478 & -0.227 & 0.008 & 0.003 & -0.04 & 0.085 & 0.251 & 0.041 \\
& -0.3 & -0.211 & -0.213 & -0.25 & -0.09 & 0.06 & 0.048 & -0.13 & -0.286 & -0.07 & -0.121 & 0.213 & -0.179 & -0.057 & -0.036 & -0.025
\end{tabular}

\begin{tabular}{|c|c|c|c|c|c|c|c|c|c|c|c|c|c|c|c|c|}
\hline \multirow[t]{16}{*}{ Figure $3.8(a)$} & -0.703 & 0.406 & -0.328 & \begin{tabular}{|l|}
-0.244 \\
\end{tabular} & -0.281 & 0.162 & -0.141 & 0.406 & 0.000 & -0.649 & 0.141 & -0.244 & 0.234 & 0.244 & 0.422 & 0.081 \\
\hline & -0.609 & 0.244 & -0.375 & -0.162 & -0.234 & 0.244 & -0.047 & -0.731 & 0.047 & -0.568 & 0.094 & -0.162 & 0.187 & 0.325 & 0.375 & 0.162 \\
\hline & -0.656 & 0.325 & -0.328 & -0.081 & -0.281 & 0.325 & -0.094 & -0.649 & 0.000 & -0.487 & 0.141 & -0.081 & 0.234 & 0.406 & 0.422 & 0.244 \\
\hline & -0.609 & 0.406 & -0.375 & 0.000 & -0.234 & 0.406 & -0.047 & -0.568 & 0.047 & -0.406 & 0.094 & 0.000 & 0.281 & -0.325 & 0.375 & 0.325 \\
\hline & -0.516 & 0.081 & -0.328 & 0.081 & -0.141 & -0.568 & -0.094 & -0.325 & 0.047 & -0.244 & 0.141 & 0.081 & 0.328 & -0.244 & 0.422 & 0.406 \\
\hline & -0.562 & 0.162 & -0.375 & 0.162 & -0.187 & -0.487 & -0.047 & -0.244 & 0.000 & -0.162 & 0.094 & 0.162 & 0.281 & -0.162 & 0.469 & 0.000 \\
\hline & -0.516 & 0.244 & -0.328 & 0.244 & -0.141 & -0.406 & -0.094 & -0.162 & 0.047 & -0.081 & 0.094 & 0.325 & 0.328 & -0.081 & 0.516 & 0.081 \\
\hline & -0.562 & 0.325 & -0.375 & 0.325 & -0.187 & -0.325 & -0.047 & -0.081 & 0.047 & 0.081 & 0.141 & 0.406 & 0.281 & 0.000 & 0.469 & 0.162 \\
\hline & -0.516 & 0.406 & -0.328 & 0.406 & -0.141 & -0.244 & -0.094 & 0.000 & 0.047 & 0.244 & 0.187 & -0.487 & 0.328 & 0.081 & 0.516 & 0.244 \\
\hline & -0.422 & -0.081 & -0.234 & -0.406 & -0.187 & -0.162 & -0.047 & 0.081 & 0.000 & 0.325 & 0.234 & -0.406 & 0.281 & 0.162 & 0.469 & 0.325 \\
\hline & -0.469 & 0.000 & -0.281 & -0.325 & -0.141 & -0.081 & -0.094 & 0.162 & 0.047 & 0.406 & 0.187 & -0.325 & 0.328 & 0.244 & 0.562 & 0.162 \\
\hline & -0.422 & 0.081 & -0.234 & -0.244 & -0.187 & 0.000 & -0.047 & 0.244 & 0.094 & -0.649 & 0.234 & -0.244 & 0.281 & 0.325 & 0.609 & 0.244 \\
\hline & -0.469 & 0.162 & -0.281 & -0.162 & -0.141 & 0.081 & -0.094 & 0.325 & 0.141 & -0.568 & 0.187 & -0.162 & 0.328 & 0.406 & 0.562 & 0.325 \\
\hline & -0.422 & 0.244 & -0.234 & -0.081 & -0.187 & 0.162 & -0.047 & 0.406 & 0.094 & -0.487 & 0.187 & 0.000 & 0.375 & -0.162 & 0.609 & 0.406 \\
\hline & -0.469 & 0.325 & -0.281 & 0.000 & -0.141 & 0.244 & 0.000 & -0.812 & 0.141 & -0.406 & 0.234 & 0.081 & 0.422 & -0.081 & 0.656 & 0.325 \\
\hline & -0.422 & 0.406 & -0.234 & 0.081 & -0.187 & 0.325 & 0.047 & $|-0.731|$ & 0.094 & -0.325 & 0.187 & 0.162 & 0.375 & 0.000 & 0.703 & 0.406 \\
\hline \multirow[t]{16}{*}{ Figure $3.8(\mathrm{~b})$} & -0.470 & 0.251 & -0.181 & \begin{tabular}{|l|}
-0.376 \\
\end{tabular} & -0.145 & -0.188 & -0.072 & \begin{tabular}{|l|}
-0.063 \\
\end{tabular} & 0.036 & 0.125 & 0.145 & -0.313 & 0.253 & 0.125 & 0.398 & 0.251 \\
\hline & -0.398 & 0.125 & -0.217 & -0.313 & -0.109 & -0.125 & -0.036 & 0.000 & 0.000 & 0.188 & 0.181 & -0.251 & 0.217 & 0.188 & 0.362 & 0.313 \\
\hline & -0.434 & 0.188 & -0.181 & -0.251 & -0.145 & -0.063 & -0.072 & 0.063 & 0.036 & 0.251 & 0.145 & -0.188 & 0.253 & 0.251 & 0.434 & 0.063 \\
\hline & -0.398 & 0.251 & -0.217 & -0.188 & -0.109 & 0.000 & -0.036 & 0.125 & 0.000 & 0.313 & 0.181 & -0.125 & 0.217 & 0.313 & 0.470 & 0.125 \\
\hline & -0.326 & 0.000 & -0.181 & -0.125 & -0.145 & 0.063 & -0.072 & 0.188 & 0.109 & -0.376 & 0.145 & -0.063 & 0.289 & -0.063 & 0.434 & 0.188 \\
\hline & -0.362 & 0.063 & -0.217 & -0.063 & -0.109 & 0.125 & -0.036 & 0.251 & 0.072 & -0.313 & 0.181 & 0.000 & 0.326 & 0.000 & 0.470 & 0.251 \\
\hline & -0.326 & 0.125 & -0.181 & 0.000 & -0.145 & 0.188 & -0.072 & 0.313 & 0.109 & -0.251 & 0.145 & 0.063 & 0.289 & 0.063 & 0.434 & 0.313 \\
\hline & -0.362 & 0.188 & -0.217 & 0.063 & -0.109 & 0.251 & 0.000 & -0.439 & 0.072 & -0.188 & 0.181 & 0.125 & 0.326 & 0.125 & 0.506 & 0.188 \\
\hline & -0.326 & 0.251 & -0.181 & 0.125 & -0.072 & -0.564 & 0.036 & -0.376 & 0.109 & -0.125 & 0.145 & 0.188 & 0.289 & 0.188 & 0.543 & 0.251 \\
\hline & -0.253 & -0.125 & -0.217 & 0.188 & -0.036 & -0.501 & 0.000 & -0.313 & 0.072 & -0.063 & 0.181 & 0.251 & 0.326 & 0.251 & 0.506 & 0.313 \\
\hline & -0.289 & -0.063 & -0.181 & 0.251 & -0.072 & -0.439 & 0.036 & -0.251 & 0.109 & 0.000 & 0.145 & 0.313 & 0.289 & 0.313 & 0.543 & 0.376 \\
\hline & -0.253 & 0.000 & -0.109 & -0.501 & -0.036 & -0.376 & 0.000 & -0.188 & 0.072 & 0.063 & 0.217 & -0.188 & 0.362 & -0.063 & 0.615 & 0.251 \\
\hline & -0.289 & 0.063 & -0.145 & -0.439 & -0.072 & -0.313 & 0.036 & -0.125 & 0.109 & 0.125 & 0.253 & -0.125 & 0.398 & 0.000 & 0.579 & 0.313 \\
\hline & -0.253 & 0.125 & -0.109 & -0.376 & -0.036 & -0.251 & 0.000 & -0.063 & 0.072 & 0.188 & 0.217 & -0.063 & 0.362 & 0.063 & 0.615 & 0.376 \\
\hline & -0.289 & 0.188 & -0.145 & -0.313 & -0.072 & -0.188 & 0.036 & 0.000 & 0.109 & 0.251 & 0.253 & 0.000 & 0.398 & 0.125 & 0.651 & 0.313 \\
\hline & -0.253 & 0.251 & -0.109 & -0.251 & -0.036 & -0.125 & 0.000 & 0.063 & 0.072 & 0.313 & 0.217 & 0.063 & 0.362 & 0.188 & 0.687 & 0.376 \\
\hline
\end{tabular}




\begin{tabular}{l|l|l|l|} 
& $\mathrm{R}$ & $\mathrm{G}$ & $\mathrm{B}$ \\
\hline Figure 3.9 (a) & 0.000 & 1.100 & 0.000 \\
& 0.000 & 0.000 & 1.100 \\
& 0.000 & 0.384 & 0.716 \\
& 0.550 & 0.550 & 0.000 \\
& 0.061 & 0.611 & 0.228 \\
& 1.100 & 0.000 & 0.000 \\
& 0.611 & 0.061 & 0.228 \\
& 0.384 & 0.000 & 0.716
\end{tabular}

Figure $3.11(a)$

\begin{tabular}{|l|l|l|}
1.100 & 0.000 & 0.000 \\
\hline
\end{tabular} $\begin{array}{lllll}0.722 & 0.168 & 0.210\end{array}$ \begin{tabular}{llll}
0.227 & 0.409 & 0.453 \\
\hline
\end{tabular} \begin{tabular}{llll}
0.516 & 0.130 & 0.255 \\
\hline
\end{tabular} $\begin{array}{lllll}0.583 & 0.309 & 0.207\end{array}$ $\begin{array}{lllll}0.000 & 0.961 & 0.139\end{array}$ \begin{tabular}{ll|l|l|l|l|l|l|l|l|l|l|l|l|l|}
0.825 & 0.275 & 0.000
\end{tabular} \begin{tabular}{ll|l|l|l|l|l|l|l|l|l|l|l|}
0.444 & 0.448 & 0.208 \\
\hline
\end{tabular} $\begin{array}{lllll}0.055 & 0.792 & 0.053\end{array}$ $\begin{array}{llll}0.962 & 0.138 & 0.000\end{array}$ \begin{tabular}{ll|l|l|}
0.412 & 0.688 & 0.000 \\
\hline
\end{tabular} \begin{tabular}{llll}
0.687 & 0.413 & 0.000 \\
\hline
\end{tabular} $\begin{array}{llll}0.008 & 0.626 & 0.266\end{array}$ $\begin{array}{llll}0.305 & 0.588 & 0.206\end{array}$ $\begin{array}{lllll}0.777 & 0.084 & 0.040\end{array}$ \begin{tabular}{ll|l|l|}
0.039 & 0.308 & 0.554 \\
\hline
\end{tabular} \begin{tabular}{llll}
0.137 & 0.963 & 0.000 \\
\hline
\end{tabular} \begin{tabular}{ll|l|l|l|l|l|l|l|l|l|l|l|l|}
0.000 & 0.497 & 0.603 \\
\hline
\end{tabular} \begin{tabular}{lllll}
0.550 & 0.550 & 0.000 \\
\hline
\end{tabular} $\begin{array}{llll}0.220 & 0.642 & 0.039\end{array}$ \begin{tabular}{lllll}
0.499 & 0.364 & 0.037 \\
\hline
\end{tabular} $\begin{array}{lllll}0.164 & 0.731 & 0.205 \\ 0.000\end{array}$ $\begin{array}{lllll}0.000 & 1.100 & 0.000\end{array}$ \begin{tabular}{ll|l|l|l|l|l|l|l|l|l|l|}
0.275 & 0.825 & 0.000 \\
\hline
\end{tabular} \begin{tabular}{ll|l|l|l|l|l|l|l|l|l|l|l|l|}
0.235 & 0.411 & 0.255 \\
0
\end{tabular} $\begin{array}{llll}0.638 & 0.224 & 0.038\end{array}$ \begin{tabular}{llll|l|l|l|l|l|l|l|l|l|l|l|l|}
0.360 & 0.502 & 0.038 \\
0.374 & 0.270 & 0.256
\end{tabular} $\begin{array}{llll}0.374 & 0.270 & 0.256\end{array}$ \begin{tabular}{llll}
0.000 & 0.638 & 0.462 \\
\hline
\end{tabular} $\begin{array}{llll}0.175 & 0.572 & 0.353\end{array}$ \begin{tabular}{|l|l|l|l|l|l|l|l|l|l|l|l|}
0.035 & 0.449 & 0.415 \\
\hline
\end{tabular} $\mid$\begin{tabular}{ll|l|l|l|}
0.000 & 0.822 & 0.278
\end{tabular}

\begin{tabular}{l|l|l|l|} 
& $\mathrm{R}$ & $\mathrm{G}$ & $\mathrm{B}$ \\
\hline Figure 3.10 (a) & 1.100 & 0.000 & 0.000 \\
& 0.722 & 0.168 & 0.210 \\
& 0.227 & 0.409 & 0.453 \\
& 0.516 & 0.130 & 0.255 \\
& 0.583 & 0.309 & 0.207 \\
& 0.000 & 0.961 & 0.139 \\
& 0.825 & 0.275 & 0.000 \\
& 0.444 & 0.448 & 0.208 \\
& 0.055 & 0.792 & 0.053 \\
& 0.962 & 0.138 & 0.000 \\
& 0.412 & 0.688 & 0.000 \\
& 0.687 & 0.413 & 0.000 \\
& 0.008 & 0.626 & 0.266 \\
& 0.305 & 0.588 & 0.206 \\
& 0.777 & 0.084 & 0.040 \\
& 0.039 & 0.308 & 0.554 \\
& 0.137 & 0.963 & 0.000 \\
& 0.000 & 0.497 & 0.603 \\
& 0.550 & 0.550 & 0.000 \\
& 0.220 & 0.642 & 0.039 \\
& 0.499 & 0.364 & 0.037 \\
& 0.164 & 0.731 & 0.205 \\
& 0.000 & 1.100 & 0.000 \\
& 0.275 & 0.825 & 0.000 \\
& 0.235 & 0.411 & 0.255 \\
& 0.638 & 0.224 & 0.038 \\
& 0.360 & 0.502 & 0.038 \\
0.374 & 0.270 & 0.256 \\
& 0.000 & 0.638 & 0.462 \\
0.175 & 0.572 & 0.353 \\
& 0.035 & 0.449 & 0.415 \\
0.000 & 0.822 & 0.278
\end{tabular}




\section{A.2 Constellations from Chapter 5}

This section contains the digital representations of the constellations depicted in Figures 5.2, 5.3, 5.4, and 5.5.

\begin{tabular}{|c|c|c|c|c|c|c|c|c|c|c|c|c|c|c|c|}
\hline & $\mathrm{R}$ & G & B & & $\mathrm{R}$ & G & B & & $\mathrm{R}$ & G & B & & $\mathrm{R}$ & G & \\
\hline $2700 \mathrm{~K} 8-\mathrm{CSK}$ & 121 & 135 & 0 & 3500K 8-CSK & 168 & 87 & $\overline{0}$ & 4000K 8-CSK & 157 & 99 & 0 & 4500K 8-CSK & 112 & 98 & \\
\hline & 154 & 102 & 0 & & 99 & 47 & 109 & & 124 & 41 & 90 & & 255 & 0 & \\
\hline & 255 & 0 & 0 & & 214 & 41 & 0 & & 110 & 145 & 0 & & 204 & & \\
\hline & 187 & 69 & 0 & & 140 & 0 & 116 & & 209 & 47 & 0 & & 146 & & \\
\hline & 163 & 16 & 76 & & 182 & 0 & 74 & & 255 & 0 & 0 & & 165 & 91 & \\
\hline & 223 & 32 & 0 & & 125 & 105 & 25 & & 82 & 11 & 162 & & 70 & 183 & \\
\hline & 132 & 59 & 65 & & 256 & 0 & 0 & & 172 & 0 & 83 & & 53 & 87 & \\
\hline & 197 & 0 & 58 & & 88 & 167 & 0 & & 68 & 114 & 73 & & 94 & 0 & \\
\hline
\end{tabular}

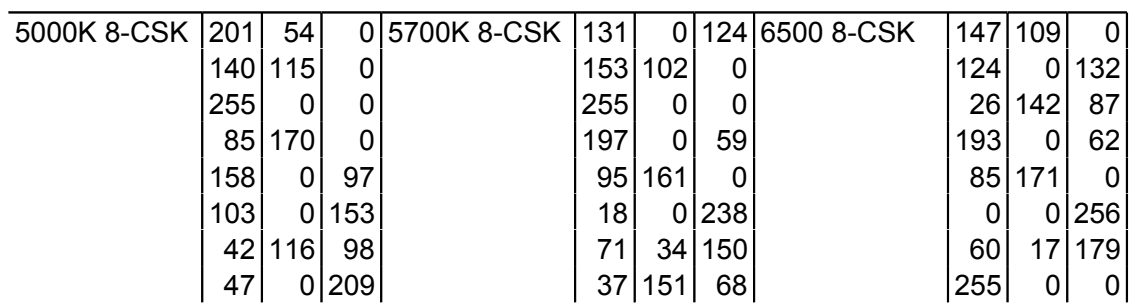




\begin{tabular}{l|r|r|r|l|r|r|r|l|r|r|r|} 
& $\mathrm{R}$ & $\mathrm{G}$ & $\mathrm{B}$ & & $\mathrm{R}$ & $\mathrm{G}$ & $\mathrm{B}$ & & $\mathrm{R}$ & $\mathrm{G}$ & $\mathrm{B}$ \\
\hline IEEE 4-CSK & 255 & 0 & 0 & Optimal 4-CSK & 244 & 6 & 6 & $3000 \mathrm{~K}$ 4-CSK & 104 & 152 & 0 \\
& 0 & 255 & 0 & & 114 & 77 & 65 & & 255 & 0 & 0 \\
& 0 & 0 & 255 & & 0 & 255 & 0 & & 184 & 60 & 2 \\
& 85 & 85 & 85 & & 0 & 0 & 255 & & 126 & 0 & 129
\end{tabular}

\begin{tabular}{|c|c|c|c|c|c|c|c|c|c|c|c|}
\hline IEEE 8-CSK & 128 & 128 & 0 & Optimal 8-CSK & 255 & 0 & 0 & 3000K 8-CSK & 221 & 0 & 35 \\
\hline & 0 & 255 & 0 & & 0 & 0 & 255 & & 178 & 0 & 77 \\
\hline & 255 & 0 & 0 & & 189 & 0 & 66 & & 188 & 67 & 0 \\
\hline & 157 & 28 & 69 & & 23 & 117 & 115 & & 150 & 105 & 0 \\
\hline & 0 & 0 & 255 & & 90 & 36 & 129 & & 112 & 143 & 0 \\
\hline & 87 & 0 & 168 & & 140 & 115 & 0 & & 140 & 38 & 77 \\
\hline & 28 & 157 & 69 & & 75 & 181 & 0 & & 255 & 0 & 0 \\
\hline & 0 & 87 & $|168|$ & & 0 & 255 & 0 & & 95 & 88 & 73 \\
\hline
\end{tabular}

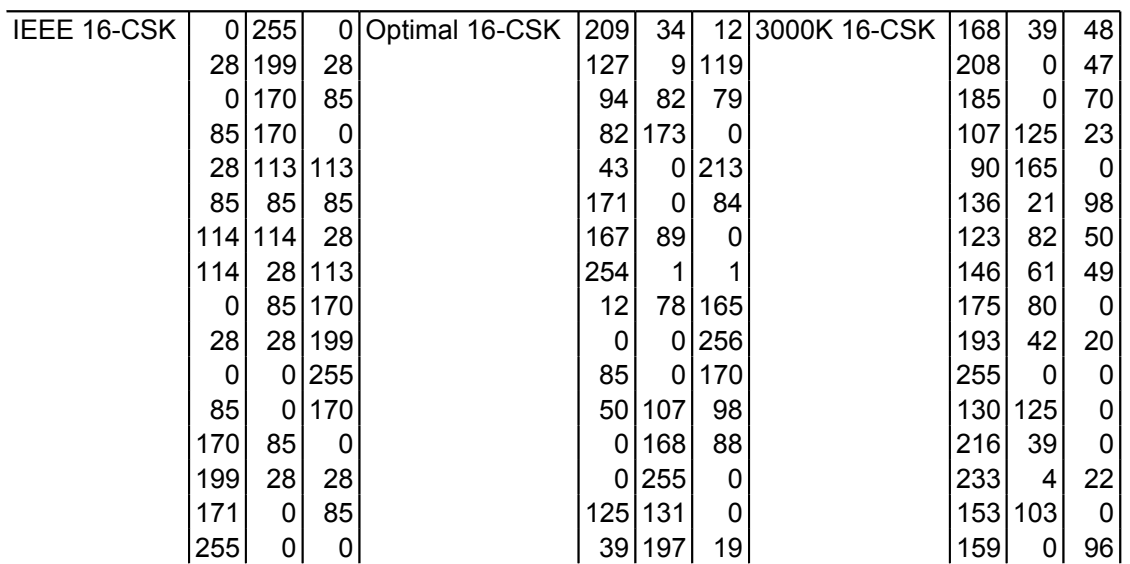




\begin{tabular}{|c|c|c|c|c|c|c|c|c|c|c|c|c|}
\hline & $\mathrm{R}$ & G & $\mathrm{B}$ & $\mathrm{R}$ & G & $B$ & $\mathrm{R}$ & G & $\mathrm{B}$ & $\mathrm{R}$ & G & B \\
\hline \multirow[t]{8}{*}{ Optimal 32-CSK } & 0 & 0 & 255 & 27 & 156 & 72 & 87 & 0 & 168 & 179 & 51 & 25 \\
\hline & 115 & 0 & 140 & 60 & 0 & 195 & 52 & 203 & 0 & 45 & 100 & 111 \\
\hline & 0 & 143 & 112 & 57 & 145 & 53 & 131 & 124 & 0 & 95 & 51 & 109 \\
\hline & 144 & 55 & 56 & 256 & 0 & 0 & 0 & 57 & 199 & 228 & 0 & 28 \\
\hline & 79 & 176 & 0 & 18 & 92 & 145 & 142 & 0 & 113 & 198 & 0 & 58 \\
\hline & 28 & 0 & 228 & 24 & 217 & 14 & 39 & 47 & 169 & 0 & 256 & 0 \\
\hline & 116 & 79 & 60 & 103 & 135 & 17 & 67 & 53 & 135 & 170 & 0 & 85 \\
\hline & 0 & 199 & 56 & 158 & 97 & 0 & 82 & 107 & 67 & 207 & 48 & 0 \\
\hline \multirow[t]{16}{*}{ Optimal 64-CSK } & 0 & 141 & 114 & 86 & 170 & 0 & 24 & 33 & \begin{tabular}{|l}
197 \\
\end{tabular} & 4 & \begin{tabular}{|l|}
177 \\
\end{tabular} & 74 \\
\hline & 18 & 102 & 135 & 37 & 100 & 118 & 237 & 18 & 0 & 120 & 34 & 101 \\
\hline & 107 & 68 & 80 & 62 & 134 & 59 & 75 & 101 & 79 & 38 & 0 & 218 \\
\hline & 163 & 70 & 23 & 223 & 0 & 33 & 11 & 66 & 178 & 31 & 67 & 158 \\
\hline & 255 & 0 & 0 & 57 & 100 & 99 & 19 & 140 & 96 & 77 & 0 & 179 \\
\hline & 152 & 0 & 104 & 126 & 69 & 61 & 171 & 0 & 85 & 57 & 0 & 198 \\
\hline & 70 & 67 & 119 & 30 & 165 & 60 & 67 & 169 & 19 & 96 & 0 & 160 \\
\hline & 18 & 199 & 38 & 50 & 67 & 138 & 0 & 0 & 256 & 113 & 103 & 40 \\
\hline & 100 & 137 & 19 & 217 & 38 & 0 & 83 & 33 & 139 & 63 & 33 & 159 \\
\hline & 204 & 17 & 35 & 189 & 0 & 66 & 19 & 0 & 237 & 49 & 168 & 39 \\
\hline & 0 & 39 & 216 & 44 & 133 & 79 & 0 & 103 & 153 & 81 & 135 & 39 \\
\hline & 150 & 105 & 0 & 0 & 256 & 0 & 102 & 34 & 120 & 118 & 137 & 0 \\
\hline & 94 & 102 & 59 & 199 & 57 & 0 & 18 & 237 & 0 & 88 & 67 & 99 \\
\hline & 144 & 69 & 42 & 132 & 104 & 20 & 181 & 75 & 0 & 35 & 211 & 10 \\
\hline & 133 & 0 & 122 & 0 & 217 & 38 & 178 & 37 & 41 & 158 & 35 & 63 \\
\hline & 54 & 202 & 0 & 115 & 0 & 141 & 139 & 34 & 82 & 44 & 33 & 178 \\
\hline
\end{tabular}




\section{Appendix B}

\section{Calculating Correlated Color}

\section{Temperature}

The method, as defined by the CIE, for calculating the CCT of an SPD requires knowledge of the chromaticity of a block body radiator over temperature, in Kelvin, and the $x y$ chromaticity of the luminary. The $x y$ chromaticities are then converted to a new color space, termed the $u v$ chromaticity space, using the transforms shown in (B.1).

$$
\begin{aligned}
& u=\frac{4 x}{-2 x+12 y+3} \\
& v=\frac{6 y}{-2 x+12 y+3}
\end{aligned}
$$

. The $x y$ chromaticity coordinates of a black body radiator are collectively known as the Planckian locus, whose elements are identified by calculating the $x y$ chromaticity of the following SPD,

$$
P(\lambda)=\frac{2 \pi h c^{2}}{\lambda^{5}} \frac{1}{\exp \left(\frac{h c}{\lambda k T}\right)-1}
$$


where $T$ is the temperature of the black body, and $h, c$ and $k$ are Plank's constant, the speed of light and Boltzmann's constant respectively.

The CCT is calculated by determined the temperature of the black body radiator whose $u v$ chromaticity coordinates have the minimum euclidean distance from the source $u v$ chromaticity coordinates. 